\title{
HELSINKI UNIVERSITY OF TECHNOLOGY
}

Faculty of Electronics, Communications and Automation

Master's Programme in Communications Engineering

Zheng Chang

\section{OFDM Interference Analysis with 'Dirty RF'}

ESPOO, May 2009.

Thesis submitted in partial fulfilment of the requirements of the degree of Master of Science in Technology.

Supervisor : Prof. Olav Tirkkonen

Instructor : Ph.D. Natalia Ermolova

Department of Communications and Networking

Espoo 2009 
HELSINKI UNIVERSITY OF

ABSTRACT OF

TECHNOLOGY

Faculty of Electronics, Communications and Automation

- Master's Programme in Communications Engineering -

\begin{tabular}{|ll|}
\hline $\begin{array}{l}\text { Author: } \\
\text { Title of thesis: } \\
\text { OFDM Interference Analysis with 'Dirty RF' }\end{array}$ \\
\\
\end{tabular}

When passing through the RF analog electronics devices of the transceiver, the information signal is subject to various distortions named 'dirty RF' effects. Some distortions are inherent to analog radio devices, such as high power amplifiers (HPA) at the transmitter and DAC/ADC. Others, such as phase noise and I/Q imbalance, result from various impairments in the transceiver RF front end devices. Regardless of the nature, each factor degrades the communication system performance.

Owning to a number of advantages, such as the ability to provide a high data rate in a fading environment and relatively simple structure of the frequency domain equalizer, OFDM is considered as a strong candidate for future wireless broadband systems. OFDM performance, however, is very sensitive to the above $R F$ impairments, such as nonlinear distortions from HPA, I/Q imbalance, phase noise etc.

While inherently observed in any transceiver, in the most of part the dirty RF effects so far have been analyzed separately and techniques for their mitigation have been elaborated separately too. Meanwhile, when affecting jointly the above impairments may strengthen each other and moreover, techniques for mitigation of one factor may enhance the impact of another one.

The aim of this work is creating a simulation model and analyzing OFDM where I/Q imbalance, HPA, and phase noise effects are jointly taken into account.

Keywords: OFDM, dirty RF, I/Q imbalance, phase noise, power amplifier.

Language: English 
Elektroniikan, tietoliikenteen ja automaation osasto

\begin{tabular}{|lll|}
\hline $\begin{array}{l}\text { Tekijä: } \\
\text { Työn nimi: } \\
\text { OFDM Interference Analysis with 'Dirty RF' }\end{array}$ \\
\hline Päiväys: & 18. maaliskuuta 2009 & Sivumäärä: $14+89$ \\
\hline Professuuri: & Tietoliikennetekniikka & Koodi: S-72 \\
\hline $\begin{array}{l}\text { Työn valvoja: } \\
\text { Työn ohjaaja: }\end{array}$ & Trof. Olav Tirkkonen Natalia Ermolova & \\
\hline
\end{tabular}

Kulkiessaan läpi lähetin-vastaanottimen analogisten RF-osien, informaatiosignaali altistuu lukuisille vääristymille, joita kutsutaan Ťdirty RF ̌̌ -ilmiöiksi. Jotkin vääristymät ovat luontaisia analogisille radiolaitteille, kuten tehovahvistimille (HPA) lähettimessä ja DA/AD -muuntimille. Toiset, kuten vaihekohina ja I/Q-epätasapaino ovat puolestaan seurausta lähetin-vastaanottimen RF-etupään aiheuttamasta signaalin laadun heikkenemisestä. Riippumatta vääristymän aiheuttajasta, kukin edellä mainittu tekijä alentaa tietoliikennejärjestelmän suorituskykyä.

Johtuen lukuisista eduistaan, kuten kyvystään tarjota korkeita datanopeuksia häipyvässä ympäristössä sekä verrattain yksinkertaisesta taajuusalueen ekvalisaattorin rakenteesta, OFDM:ää pidetään vahvana ehdokkaana tulevaisuuden langattomiin laajakaistajärjestelmiin. OFDM:n suorituskyky on kuitenkin erittäin herkkä yllä mainitulle RFosien aiheuttamalle signaalin laadun heikkenemiselle, kuten tehovahvistimen, I/Q-epätasapainon, vaihekohinan jne. aiheuttamille epälineaarisille vääristymille.

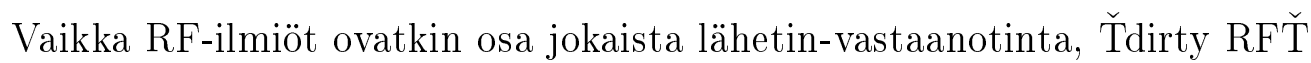
-ilmiöitä kuten myös menetelmiä niiden vähentämiseksi, on analysoitu yksittäin, muista irrallaan. Kuitenkin, vaikuttaessaan yhtäaikaa, em. ilmiöt voivat vahvistaa toistaan ja lisäksi yhden ilmiön heikentämiseen tähtäävän menetelmän käyttö voi vahvistaa toista ilmiötä.

Tämän työn tavoitteena on luoda simulaatiomalli ja analysoida OFDM:ää, kun I/Q-epätasapaino-, tehovahvistin- ja vaihekohina-ilmiöt otetaan samanaikaisesti huomioon.

$\begin{array}{ll}\text { Avainsanat: } & \text { OFDM, dirty RF, I/Q-epätasapaino } \\ & \text { tehovahvistin- ja vaihekohina-ilmiöt } \\ \text { Kieli: } & \text { Englanti }\end{array}$




\section{Acknowledgements}

This thesis is based on the work that was carried out in the Communications Laboratory, TKK, from September 2008 to May 2009. The thesis is part of the project named:" DIRTY-RF: Advanced Techniques for RF Impairment Mitigation in Future Wireless Radio Systems ". The project is funded by Tekes.

First, I wish to express my sincere gratitude to my thesis supervisor Prof. Olav Tirkkonen and instructor Ph.D. Natalia Ermolova for giving me this opportunity to carry out this work. I would like to thank Prof. Olav Tirkkonen for his

continuous encouragement, professional advices, and constant support during the course of this thesis. I would like to thank Ph.D. Natalia Ermolova for her patience, her specific guidance, and her interest shown to my thesis work. The discussions I had with Prof. Olav Tirkkonen and Ph.D. Natalia Ermolova, their comments and suggestions have greatly contributed to the quality of the thesis. I also wan to thank William Martin from the faculty for his English language support.

I like to thank my colleagues M.Sc Udesh Oruthota, M.Sc Lu Wei and Zhong Zheng for their help during the work. I also would like to thank all my friends in Finland and in China, for their genuine friendship.

Finally, I haven't got enough words for thanking my parents for their love, support, and encouragement during my whole life.

Espoo, May 2009

Zheng Chang 


\section{List of Abbreviations}

\begin{tabular}{|c|c|}
\hline OFDM & Orthogonal Frequency Division Multiplexing \\
\hline $\mathrm{DAB}$ & Digital Audio Broadcasting \\
\hline DVB & Digital Video Broadcasting \\
\hline DSL & Digital Subscriber Lines \\
\hline WLANs & Wireless Local Area Networks \\
\hline HIPERLAN $/ 2$ & $\begin{array}{l}\text { European high Performance Local Area Net- } \\
\text { work }\end{array}$ \\
\hline WiMAX & $\begin{array}{l}\text { Worldwide Interoperability for Microwave Ac- } \\
\text { cess }\end{array}$ \\
\hline PAPR & Peak-to-Average Power Ratio \\
\hline PA & Power Amplifier \\
\hline $\mathrm{I} / \mathrm{Q}$ & In-phase and Quadrature \\
\hline MCM & Multicarrier Modulation \\
\hline QAM & Quadrature Amplitude Modulation \\
\hline PSK & Phase Shift Keying \\
\hline FFT & Fast Fourier Transform \\
\hline IFFT & Inverse Fast Fourier Transform \\
\hline ISI & Intersymbol Interference \\
\hline ICI & Intercarrier Interference \\
\hline WSSUS & Wide Sense Stationary Uncorrelated Scattering \\
\hline GI & Guard Interval \\
\hline $\mathrm{CP}$ & Cyclic Prefix \\
\hline FEC & Forward Error Correction \\
\hline
\end{tabular}




\begin{tabular}{|c|c|}
\hline $\mathrm{S} / \mathrm{P}$ & Serial to Parallel \\
\hline $\mathrm{DAC}$ & Digital to Analog Converter \\
\hline SNR & Signal to Noise Ratio \\
\hline $\mathrm{DFT}$ & Discrete Fourier Transform \\
\hline $\mathrm{CPE}$ & Common Phase Error \\
\hline MMSE & Minimum Mean Squire Estimation \\
\hline AM-AM & Amplitude-to-Amplitude Distortion \\
\hline AM-PM & Amplitude-to-Phase Distortion \\
\hline IBO & Input Back-off \\
\hline $\mathrm{OBO}$ & Output Back-off \\
\hline SEL & Soft Envelope Limiter \\
\hline SSPA & Solid-State Power Amplifier \\
\hline TWTA & Travelling-Wave Tube Amplifier \\
\hline NLD & Nonlinear Distortion \\
\hline AWGN & Additive White Gaussian Noise \\
\hline BER & Bit Error Ratio \\
\hline $\mathrm{TD}$ & Total Degradation \\
\hline LINC & $\begin{array}{l}\text { Linear Amplification with Nonlinear Compo- } \\
\text { nents }\end{array}$ \\
\hline $\mathrm{CFO}$ & Carrier Frequency Offset \\
\hline LO & Local Oscillator \\
\hline $\mathrm{PDF}$ & Probability Density Function \\
\hline
\end{tabular}




\section{List of Symbols}

\begin{tabular}{|c|c|}
\hline$x(t)$ & Time domain OFDM signal \\
\hline$g(t)$ & Impulse Response of Pulse Shaping Filter \\
\hline$s(k)$ & Time domain QAM signal \\
\hline$T_{g}$ & Length of GI \\
\hline$T_{\text {delay }}$ & Multipath delay spread \\
\hline$r(n)$ & Samples of received signal \\
\hline$W(n)$ & Samples of noise \\
\hline$h(n)$ & Channel impulse response \\
\hline$\phi(n)$ & Phase noise \\
\hline$N_{c p}$ & Samples of CP \\
\hline$X_{k}$ & Frequency domain sample of OFDM signal \\
\hline$H_{k}$ & $\begin{array}{l}\text { Frequency domain sample of channel transfer } \\
\text { function }\end{array}$ \\
\hline$W_{k}$ & Frequency domain sample of noise \\
\hline$I_{0}$ & $\mathrm{CPE}$ \\
\hline$I_{k}$ & ICI caused by phase noise \\
\hline$\gamma_{t}$ & Amplitude imbalance at the transmitter \\
\hline$\triangle \varphi_{t}$ & Phase imbalance at the transmitter \\
\hline()$_{m}$ & Mirroring operation \\
\hline$*$ & Complex conjugate \\
\hline Pin $_{\text {sat }}$ & Input saturation power of an amplifier \\
\hline Pout $_{\text {sat }}$ & Output saturation power of an amplifier \\
\hline$P_{i n}$ ave & Average input power \\
\hline
\end{tabular}




\begin{tabular}{|c|c|}
\hline Pout $_{\text {ave }}$ & Average Output power \\
\hline$F()$ & Nonlinear function \\
\hline$F_{A}()$ & AM-AM \\
\hline$F_{P}()$ & AM-PM \\
\hline$\rho$ & Amplitude of signal \\
\hline$\psi$ & Phase of signal \\
\hline$A_{\text {sat }}$ & Input saturation voltage of the power amplifier \\
\hline$A_{o}$ & $\begin{array}{l}\text { Output saturation voltage of the power ampli- } \\
\text { fier }\end{array}$ \\
\hline$p$ & Smooth parameter of SSPA \\
\hline$\alpha$ & Shape parameter of TWTA \\
\hline$\beta$ & Shape parameter of TWTA \\
\hline$K$ & Arbitrary deterministic factor \\
\hline$d(t)$ & Additive nonlinear noise \\
\hline$\sigma_{D}^{2}$ & Variance of additive nonlinear nois \\
\hline$u(t)$ & Output signal of power amplifier \\
\hline$S N R_{\text {nonlinear }}$ & required SNR with nonlinear power amplifier \\
\hline$S N R_{\text {linear }}$ & required SNR with linear power amplifier \\
\hline$A$ & Amplifier gain in feedback system \\
\hline$G()$ & Predistortion function \\
\hline$x_{i q}(t)$ & Transmitted signal with I/Q imbalance \\
\hline$x_{d-i q}(t)$ & $\begin{array}{l}\text { Transmitted signal with } \mathrm{I} / \mathrm{Q} \text { imbalance and } \\
\text { nonlinear distortion }\end{array}$ \\
\hline$r_{i q-p n}(t)$ & $\begin{array}{l}\text { Received signal with } \mathrm{I} / \mathrm{Q} \text { imbalance and phase } \\
\text { noise }\end{array}$ \\
\hline$r_{p a-i q-p n}(t)$ & Received signal with dirty $\mathrm{RF}$ \\
\hline$R$ & Frequency domain received signal \\
\hline$r_{p a-i q-p n}(n)$ & Samples of received signal with dirty $R F$ \\
\hline
\end{tabular}




\section{Contents}

List of Abbreviations $\quad \mathrm{v}$

List of Symbols $\quad$ vi

1 Introduction 1

1.1 Motivation . . . . . . . . . . . . . . . . . 1

1.2 Scope of Thesis . . . . . . . . . . . . . . . 3

1.3 Contribution and Organization of Thesis . . . . . . . . . . 3

2 Basic OFDM System $\quad 4$

2.1 Orthogonal Frequency Division Multiplexing . . . . . . . . . 4

2.2 Multipath Channel and the Use of Cyclic Prefix (CP) . . . . 6

2.2.1 Multipath Channel Model . . . . . . . . . . . 6

2.2.2 Cyclic Prefix . . . . . . . . . . . . . 8

2.3 Implementation of an OFDM System . . . . . . . . . . . 10

2.4 Drawbacks of OFDM . . . . . . . . . . . . . 12

2.5 Summary . . . . . . . . . . . . . . . . . . . 13

$\begin{array}{lll}3 & \text { Dirty RF } & 14\end{array}$

3.1 Phase Noise Effects . . . . . . . . . . . . . . . . . . . . 14

3.1.1 Introduction to Phase Noise . . . . . . . . . . . . 14

3.1.2 Model of Phase Noise . . . . . . . . . . . . . . . . 15

3.1.3 Compensation for Phase Noise Effects . . . . . . . 18

$3.2 \mathrm{I} / \mathrm{Q}$ Imbalance Effects $\ldots \ldots \ldots \ldots$ 
3.2.1 Introduction to I/Q Imbalance . . . . . . . . . . . . . 18

3.2.2 Model of I/Q Imbalance in OFDM . . . . . . . . . . . . 19

3.2.3 Compensation for I/Q Imbalance Effects . . . . . . . . . 21

3.3 Nonlinear Power Amplifier . . . . . . . . . . . . . . . . . 22

3.3.1 Characteristics of Nonlinear Power Amplifier . . . . . . . 22

3.3.2 PAPR Problem in OFDM . . . . . . . . . . . . 24

3.3.3 Frequently Used Models of Nonlinear Power Amplifier . . 25

3.3.4 Effects of Nonlinearity on OFDM Signal . . . . . . 27

3.3.5 Nonlinear Distortion Cancellation Technique . . . . . . . 32

3.4 Other Impairments . . . . . . . . . . . . . . . . . . . 36

$3.4 .1 \quad$ Jitter Effects . . . . . . . . . . . . 36

3.4.2 Carrier Frequency Offsets . . . . . . . . . . 37

3.5 Summary . . . . . . . . . . . . . . . . . 37

4 Joint Effects of Dirty RF 38

4.1 Joint Effects . . . . . . . . . . . . . . . . . . . . . 38

4.1 .1 Introduction . . . . . . . . . . . . . 38

4.1.2 System Model of Joint Effects . . . . . . . . . . . . . 39

4.2 Simulation Results . . . . . . . . . . . . . . . . . 43

4.2.1 Simulation Description . . . . . . . . . . . . . 43

$4.2 .2 \mathrm{I} / \mathrm{Q}$ Imbalance . . . . . . . . . . . . . . . . . 44

4.2 .3 Phase Noise . . . . . . . . . . . . . . . . . . 50

4.2.4 Nonlinear Power Amplifier . . . . . . . . . . . . . . . . 55

4.2 .5 Joint Effects . . . . . . . . . . . . . . . . . . 59

4.3 Discussion and Summary . . . . . . . . . . . . . . . 74

5 Conclusions and Future Work $\quad 76$

5.1 Conclusions ..................... . . 76

5.2 Possible Future Work . . . . . . . . . . . . . . . . 77 


\section{List of Figures}

2.1 Orthogonal subcarriers in time domain . . . . . . . . . . 5

2.2 Orthogonal subcarriers in frequency domain . . . . . . . . . 6

2.3 Multipath channel ................. 7

2.4 GI $\ldots \ldots \ldots \ldots \ldots$

2.5 Cyclic extension in waveform . . . . . . . . . . . . . . 9

2.6 Cyclic extension in packet . . . . . . . . . . . . . . 10

2.7 Block diagram of the OFDM transceiver . . . . . . . . . . . 11

3.1 Effect of the phase noise on the signal constellation . . . . . . . 15

3.2 Phase noise effects on constellation, CPE dominates . . . . . . . 17

3.3 Phase noise effects on constellation, ICI dominates . . . . . . . 17

3.4 OFDM transeiver with I/Q imbalance at transmitter and receiver 19

3.5 Input/Output curve of PA . . . . . . . . . . . . . 23

3.6 AM-AM function of a SEL . . . . . . . . . . . . . 26

3.7 AM-AM function of a SSPA with different $p$ values . . . . . . 27

3.8 AM-AM function of a TWTA . . . . . . . . . . 28

3.9 AM-PM function of a TWTA . . . . . . . . . . . . . 29

3.10 Constellation of an amplified OFDM signal after SSPA with $\mathrm{OBO}=4.32 \mathrm{~dB} \ldots \ldots \ldots \ldots \ldots$

3.11 Constellation of an amplified OFDM signal after TWTA with $\mathrm{OBO}=3.24 \mathrm{~dB} \ldots \ldots \ldots \ldots \ldots \ldots$

3.12 Feedback applied around an Amplifier with Distortion . . . . . 33

3.13 Cartesian feedback model . . . . . . . . . . . . . . . 34 
3.14 Feedforward system model . . . . . . . . . . . . . . . 35

3.15 Predistortion system model . . . . . . . . . . . . . . . 35

4.1 Simplified model for OFDM transceiver . . . . . . . . . . . . 39

4.2 Block diagram of simulator . . . . . . . . . . . . . . . . 43

4.3 Effect of I/Q imbalance on the signal constellation . . . . . . . . 45

4.4 BER performance of OFDM system with $\mathrm{I} / \mathrm{Q}$ imbalance at transmitter and receiver, AWGN channel . . . . . . . . . . . 46

4.5 BER performance of OFDM system with I/Q Imbalance at transmitter and receiver, 4-tap Rayleigh fading channel . . . . 47

4.6 BER performance of OFDM system with different phase imbalance parameters, AWGN channel . . . . . . . . . . . . 47

4.7 BER performance of OFDM system with different amplitude imbalance parameters, AWGN channel . . . . . . . . . 48

4.8 Time domain histograms of interferences caused by I/Q imbal-

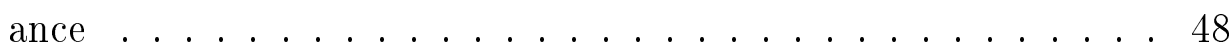

4.9 Frequency domain histograms of interferences caused by $\mathrm{I} / \mathrm{Q}$ imbalance . . . . . . . . . . . . . . . . . . 49

4.10 Frequency domain histograms of interferences caused by I/Q

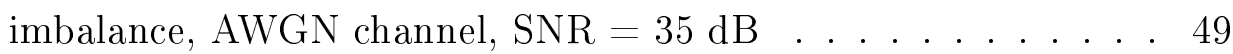

4.11 BER performance of OFDM system with phase noise, variance $=0.04$. AWGN channel . . . . . . . . . . . . 50

4.12 BER performance of OFDM system with phase noise, variance =0.04. 4-tap Rayleigh fading channel . . . . . . . . . 51

4.13 BER performance of OFDM system with phase noise, different variance, 16-QAM, AWGN channel . . . . . . . . . . . 52

4.14 Frequency domain histograms of interferences caused by phase noise, variance $=0.02 \ldots \ldots \ldots \ldots \ldots$. . . . . . . . 52

4.15 Frequency domain histograms of interferences caused by phase noise, variance $=0.2 \ldots \ldots \ldots \ldots \ldots$

4.16 Frequency domain histograms of interferences caused by phase noise, variance $=0.4 \ldots \ldots \ldots \ldots \ldots$ 
4.17 Frequency domain histograms of interferences caused by phase noise, variance $=0.6 \ldots \ldots \ldots \ldots . \ldots \ldots$

4.18 BER for OFDM system with SSPA, different OBO values, AWGN

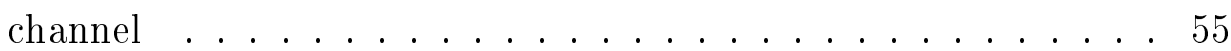

4.19 BER for OFDM system with TWTA, different OBO values,AWGN channel . . . . . . . . . . . . . . 56

4.20 Frequency domain histograms of interferences caused by SSPA, $\mathrm{OBO}=1.7 \mathrm{~dB} \ldots \ldots \ldots \ldots \ldots$

4.21 Frequency domain histograms of interferences caused by SSPA, $\mathrm{OBO}=4.2 \mathrm{~dB} \ldots \ldots \ldots \ldots \ldots \ldots$

4.22 Frequency domain histograms of interferences caused by SSPA, $\mathrm{OBO}=11.5 \mathrm{~dB} \ldots \ldots \ldots \ldots \ldots \ldots$

4.23 BER performance of OFDM system with dirty RF, AWGN channel . . . . . . . . . . . . . . . 59

4.24 BER performance of OFDM system with dirty RF, 4-tap Rayleigh fading channel . . . . . . . . . . . . . . . . 60

4.25 Constellation of OFDM signal with dirty RF . . . . . . . . . 61

4.26 BER performance of OFDM with joint effects,different I/Q imbalance parameters, 4-QAM, AWGN Channel . . . . . . . . 62

4.27 BER performance of OFDM with joint effects, different I/Q imbalance parameters, 4-QAM, 4-tap Rayleigh fading Channel . . 62

4.28 Frequency domain histograms of interferences caused by joint effects, $\triangle \varphi_{t}=\Delta \varphi_{r}=1^{\circ}, \gamma_{t}=\gamma_{r}=0.01 \ldots \ldots . \ldots .63$

4.29 Frequency domain histograms of interferences caused by joint effects, $\triangle \varphi_{t}=\Delta \varphi_{r}=3^{\circ}, \gamma_{t}=\gamma_{r}=0.03 \ldots \ldots . \ldots 63$

4.30 Frequency domain histograms of interferences caused by joint effects, $\triangle \varphi_{t}=\Delta \varphi_{r}=10^{\circ}, \gamma_{t}=\gamma_{r}=0.10 \ldots \ldots \ldots 64$

4.31 BER performance of OFDM with joint effects, different variance of phase noise, 4-QAM, AWGN channel . . . . . . . . . . . 65

4.32 BER performance of OFDM with joint effects, different variance of phase noise, 4-QAM, 4-tap Rayleigh fading Channel . . . . . 66

4.33 BER performance of OFDM with joint effects, different variance of phase noise, 16-QAM, AWGN channel . . . . . . . . . . 67 
4.34 Frequency domain histograms of interferences caused by joint effects, no phase noise . . . . . . . . . . . . . . 67

4.35 Frequency domain histograms of interferences caused by joint effects, variance of phase noise $=0.04 \ldots \ldots$. . . . 68

4.36 Frequency domain histograms of interferences caused by joint effects, variance of phase noise $=0.16 \ldots \ldots \ldots 8$

4.37 Frequency domain histograms of interferences caused by joint effects, variance of phase noise $=0.6 \ldots \ldots \ldots 69$

4.38 BER performance of OFDM with joint effects, different OBO of SSPA, 4-QAM, AWGN channel . . . . . . . . . . 70

4.39 BER performance of OFDM with joint effects ,different OBO of TWTA, 4-QAM, AWGN channel . . . . . . . . . . 71

4.40 BER performance of OFDM with joint effects, different OBO of SSPA, 4-QAM, 4-tap Rayleigh fading channel . . . . . . . . . 72

4.41 BER performance of OFDM with joint effects, different OBO of TWTA, 4-QAM, 4-tap Rayleigh fading channel . . . . . . . . 72

4.42 Frequency domain histograms of interferences caused by joint effects, $\mathrm{SSPA}, \mathrm{OBO}=1.6 \mathrm{~dB}, \ldots \ldots \ldots . \ldots 73$

4.43 Frequency domain histograms of interferences caused by joint effects, SSPA, OBO $=10.6 \mathrm{~dB}, \ldots \ldots \ldots 73$ 


\section{Chapter 1}

\section{Introduction}

\subsection{Motivation}

The wireless communication systems of the future are expected to provide higher data rates in order to meet human requirements in our modern society. The limited frequency bandwidth, which often can be seen as an obstacle to the development of telecommunication, is also the propulsion for the evaluation of the wireless technology.

In order to solve the problem of an effective use of the available spectrum, a lot of researches have been done in this area. The multicarrier technology, in particularly Orthogonal Frequency Division Multiplexing (OFDM), is an effective technique for combating channel noise, multipath effects and enabling high data rate transmissions over fading channels. OFDM has been implemented in many wired and wireless communication systems, such as Digital Audio Broadcasting (DAB) [1], Digital Video Broadcasting (DVB) [2], Digital Subscriber Lines (DSL) [3], IEEE802.11a/g Wireless Local Area Networks (WLANs) [4] [5],European high Performance Local Area Network (HIPERLAN/2) [6] and Worldwide Interoperability for Microwave Access (WiMAX).

OFDM has been seen as the core technique of the future communication systems because it has many advantages. OFDM is a special case of multicarrier modulation which makes the subcarriers orthogonal. It eliminates the interference between the subcarriers and increases the spectral efficiency of the system. By converting a single high frequency carrier to several subcarriers, OFDM enhances the ability to cope with frequency selective fading effects and narrow 
bandwidth interference. In OFDM systems, the modulation is performed on the basis of blocks with Guard Intervals (GI). A GI which makes the duration of the symbol longer can help to reduce the Intersymbol Interference(ISI) caused by the multipath fading environment.

On the other hand, the OFDM system suffers from different drawbacks. Since the OFDM signal is a combination of several modulated subcarriers, the signal may have large peak power, which makes the Peak-to-Average Power Ratio (PAPR) also large. High PAPR results in reduction of efficiency of the Power Amplifier (PA). The OFDM transmission exploits the strict orthogonality of each subcarrier, which makes OFDM sensitive to frequency offsets and phase noise. The basic modulation algorithms and other adaptive modulation techniques also increase the complexity of computations.

In the modern OFDM transceiver, the RF electronic devices have several different impairments known as "dirty RF". Some impairments that have major impacts on the system performance are:

1. Nonlinear high power amplifier: the PA in the reality behaves nonlinearly with respect to signals with amplitude variations, which causes the spectralspreading of the OFDM signal, intermodulation effects of the subcarriers and warping of the signal constellation [7].

2. Phase noise: phase noise is a random process caused by fluctuations of local oscillators. Phase noise destroys the orthogonalities among the subcarriers, causes constellation rotation and Intercarrier Interference (ICI).

3. In-phase and Quadrature (I/Q) imbalances: In practical devices, the amplitude gain and the phase gain of I and Q branch are never the same. This leads to an attenuation of the system performance.

All the impairments above deeply affect the performance of OFDM transmission. This thesis focuses on the analysis of the above impairments. Some other impairments are also reviewed. 


\subsection{Scope of Thesis}

This work considers the OFDM transmission, more precisely, the OFDM transmission with dirty RF is studied.The goal of this thesis work is to analyze the OFDM system with nonlinear PA, I/Q imbalance, and phase noise. In the past, dirty RF impairments and mitigation techniques have been analyzed mainly separately. In this thesis, the most important RF impairments are reviewed and a joint analysis of these impairments is presented. The performance is evaluated through a theoretical analysis and simulations.

\subsection{Contribution and Organization of Thesis}

This thesis work is organised as follow:

In Chapter 2, an overview of OFDM system is given. The signal model and problems associated with OFDM transmission are discussed.

Chapter 3 introduces the effects of phase noise, I/Q imbalance and nonlinear power amplifier. Characteristics as well as compensation methods of these dirty RF impairments are presented in detail. Another two impairments, jitter effects and Carrier Frequency Offsets (CFO) are described briefly as well.

Analysis of joint effects of dirty RF on OFDM is discussed in Chapter 4. Both theoretical expressions and simulation results are presented and discussed.

Finally, conclusions and possible future research directions are discussed in Chapter 5. 


\section{Chapter 2}

\section{Basic OFDM System}

This Chapter presents the basics of OFDM system. The advantages and challenges of OFDM transmission are discussed. First in Section 2.1, the concepts of OFDM are introduced. Then in Section 2.2, the properties of OFDM transmission in multipath channel are presented. In Section 2.3, OFDM transceiver technology is described. Section 2.4 briefly addresses factors that affect OFDM system performance. Section 2.5 summarizes the Chapter.

\subsection{Orthogonal Frequency Division Multiplex- ing}

The Multicarrier Modulation (MCM) is considered as an efficient way to achieve high data rate transmission because the total channel bandwidth is divided into subchannels with subcarriers and each subcarrier is modulated with a lower data rate.

OFDM is a special case of multicarrier transmission. In an OFDM system, the original data stream is split into several parallel data streams at lower data rates, and each of them is modulated separately. Conventional modulation schemes are quadrature amplitude modulation (QAM) or phase shift keying (PSK). After modulation these lower data rate streams are transmitted simultaneously through the subcarriers, resulting in achieving high-speed data transmission.

OFDM can be viewed as a form of frequency division multiplexing. In the OFDM system, all subcarriers are orthogonal to each other. OFDM allows the 
spectrum of each subcarrier to overlap, and by selecting a special set of orthogonal carrier frequencies, high spectral efficiency can be achieved because the mutual influence among the orthogonal subcarriers can be avoided. The orthogonality also greatly simplifies the design of both transmitter and receiver. A receiver can detect every subcarrier data, which commonly is done via Fast Fourier Transform (FFT). Therefore a separate filter for each subchannel is not required.

Figure 2.1 describes five orthogonal carriers in the time domain. In this example, all the subcarriers have the same amplitude and same initial phase. However, in practice, the subcarriers are modulated in different amplitude and phase. As can be seen from Figure 2.1, the orthogonality in the time domain means within an OFDM symbol period, all subcarriers have integer cycles and the numbers of the cycles between the channels differ by integer numbers.

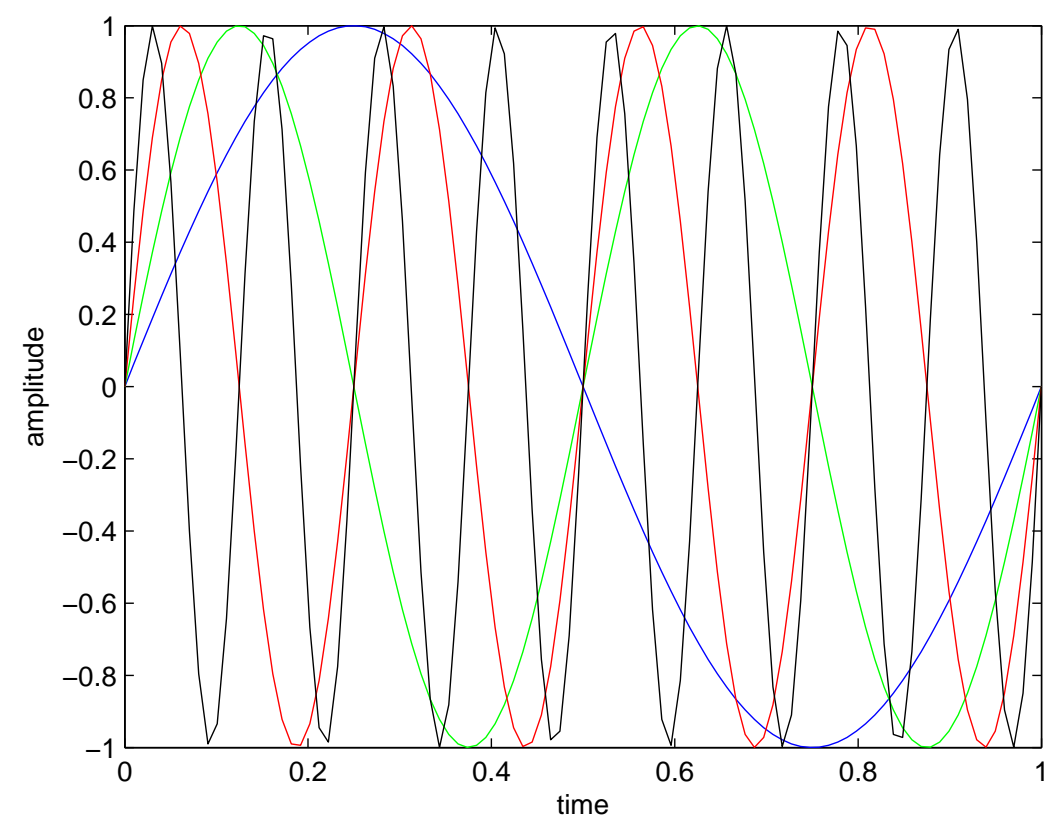

Figure 2.1: Orthogonal subcarriers in time domain

In the frequency domain, the orthogonality of the subcarriers can also be viewed as subcarriers at integer multiple copies of a single subcarrier. Figure 2.2 shows the orthogonal subcarriers in the frequency domain. 


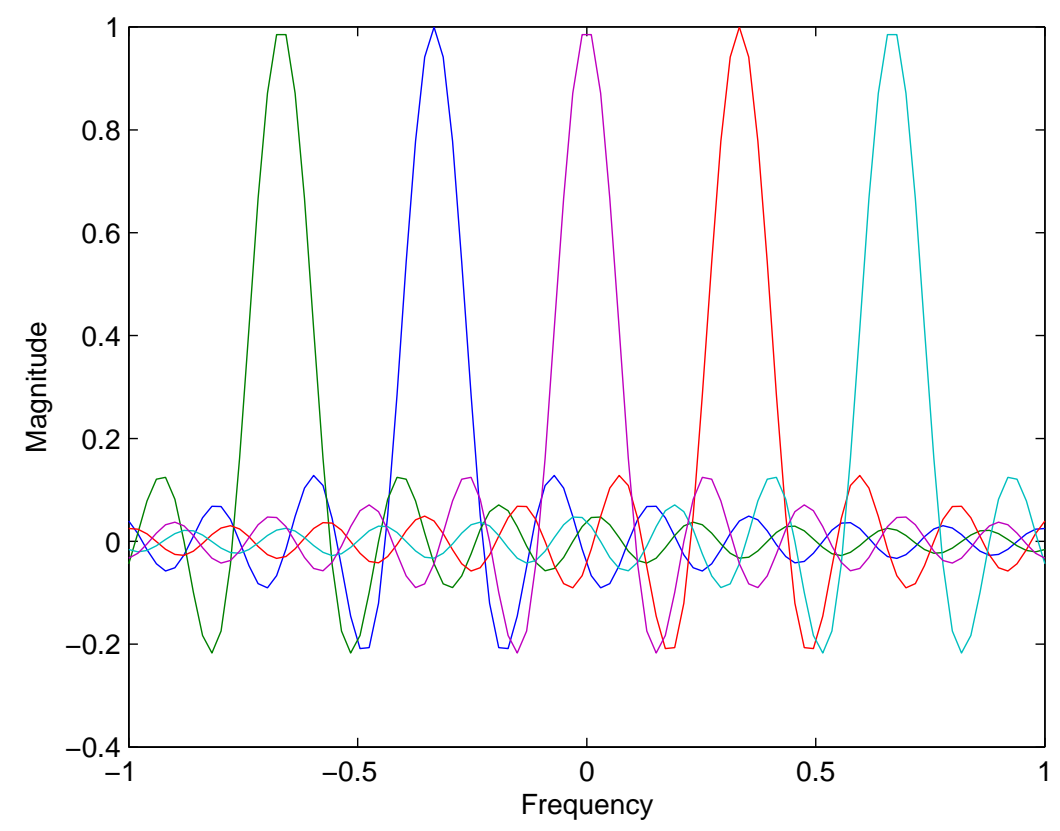

Figure 2.2: Orthogonal subcarriers in frequency domain

\subsection{Multipath Channel and the Use of Cyclic Prefix (CP)}

\subsubsection{Multipath Channel Model}

A phenomenon inherent to wireless system is the multipath channel [8]. Figure 2.3 shows a typical multipath channel.

Transmission over a multipath channel suffers from path losses, shadowing and multipath interference [9]. A multipath channel mainly causes two problems for an OFDM system. The delay spread of the multipath channel, which is defined as the propagation time difference between the longest and shortest path, causes both Intersymbol Interference (ISI) and Intercarrier Interference (ICI). ICI is also caused by Doppler spreading which is the spectrum spread caused by multipath channel and movement of the transceiver. As a result of the multipath environment, the delayed versions of the transmission signal make the received signal to be distorted. Multipath transmission also introduces fading effect which is defined as an attenuation that is caused by multipath. 


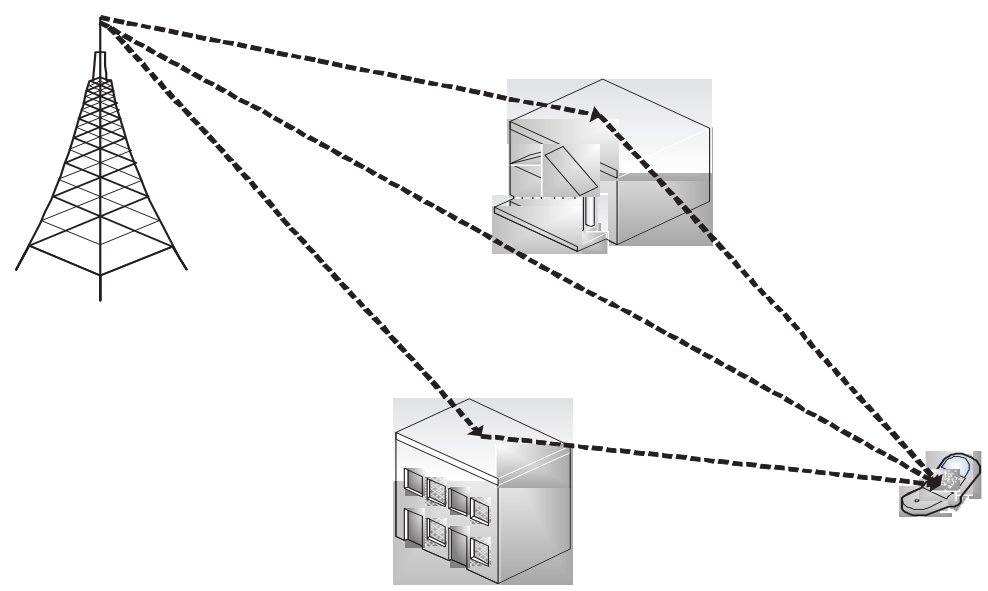

Figure 2.3: Multipath channel

To simplify the modelling of the complex and varying channel, the channel is assuming to be wide sense stationary uncorrelated scattering (WSSUS). Several parameters of the channel are presented to briefly explain channel effects, i.e. delay spread, coherence bandwidth, Doppler spread and coherence time.

The delay spread and coherence bandwidth are used to describe flatness and frequency selectivity of the channel. The coherence bandwidth which is approximately the inverse of the delay spread defines a bandwidth that the spectral components within the coherence band are correlated and therefore, the channel has same effects on these component. If the signal bandwidth is much smaller than the coherence bandwidth, the individual signal experiences flat fading where the fading depth of all frequency components is the same. Otherwise, the frequency components outside the coherence bandwidth are uncorrelated and they can experience fading which is depend on the frequency, so called frequency selective fading.

ISI and ICI are considered as major problems of OFDM transmission. These problems occur when the received OFDM symbol is distorted by the previous transmitted OFDM symbol, which is similar to a single-carrier system. One way to reduce ISI is increasing the symbol duration. When the delay spread is small compared to the symbol duration, the response of the channel cannot cause the ISI between symbols. 
On the other hand, enlarging the symbol duration can expose the signal to ICI due to the relation between the Doppler spread and coherence time of the channel. ICI is a crosstalk between subcarriers. It is a result of interference among subcarriers of a given OFDM symbol. When the coherence time, which describes the time variation of the channel impulse response and is the inverse of the Doppler spread, is shorter than symbol duration, time variations of the channel during one symbol cannot be neglected. Therefore, time variations can cause ICI since the Doppler spread is longer than the carrier spacing, resulting in spectral spreading from one subcarrier to another.

\subsubsection{Cyclic Prefix}

One of the most important reasons for choosing OFDM transmission is its efficiency to deal with the delay spread of the multipath channel. For the purpose of eliminating effects of the ISI, a guard interval(GI) is inserted between each OFDM symbol. The guard time is chosen to be longer than the expected delay spread, so that one symbol cannot interfere with the others. However, in such a case, if the GI is not chosen properly, the ISI could be increased. This effect is shown in the Figure 2.4.

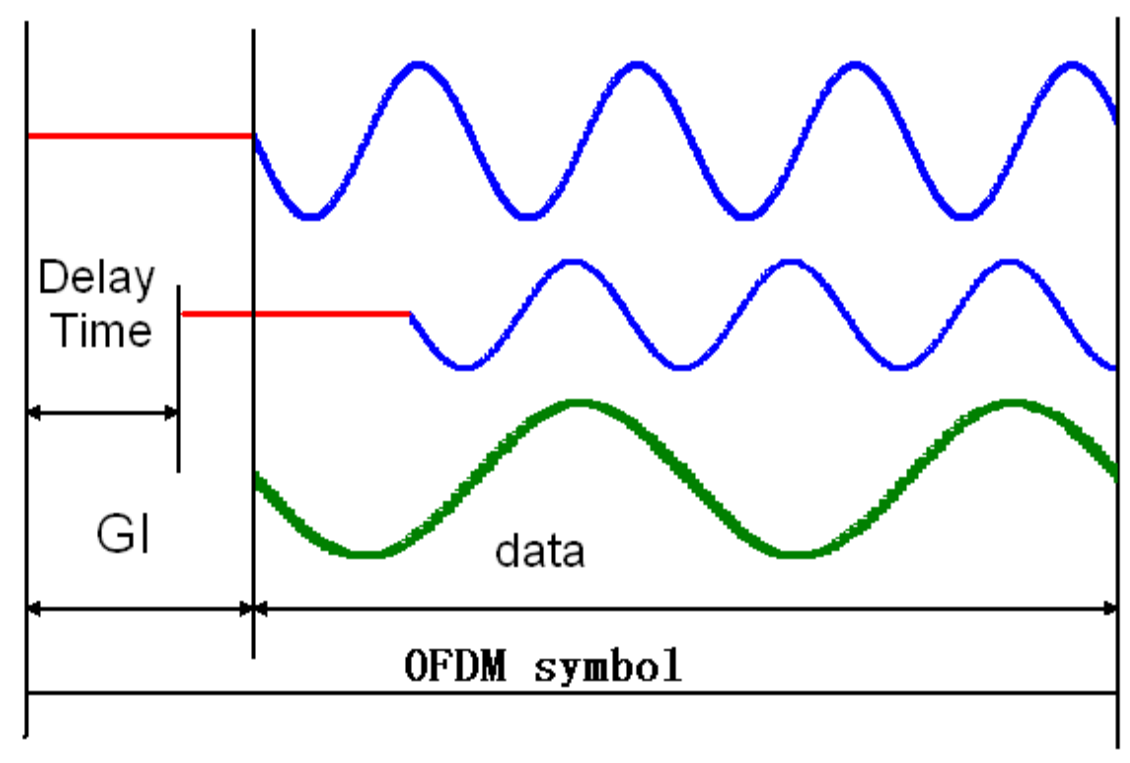

Figure 2.4: GI 
In this example, subcarrier 1 and a delayed subcarrier 2 are presented. If the receiver of the OFDM system tries to demodulate subcarrier 1 , the interference from the subcarrier 2 is taken into account. That's because within a subcarrier period, there is no integer number of periods. At the same time, there is a crosstalk between the subcarriers 1 and 2 [10].

To eliminate the ICI, cyclic extension which usually is called cyclic prefix (CP) extends the OFDM signal into the GI. Normally, this can be done by copying a part of the signal and putting it at the beginning of the signal, as shown in Figure 2.5 and 2.6.

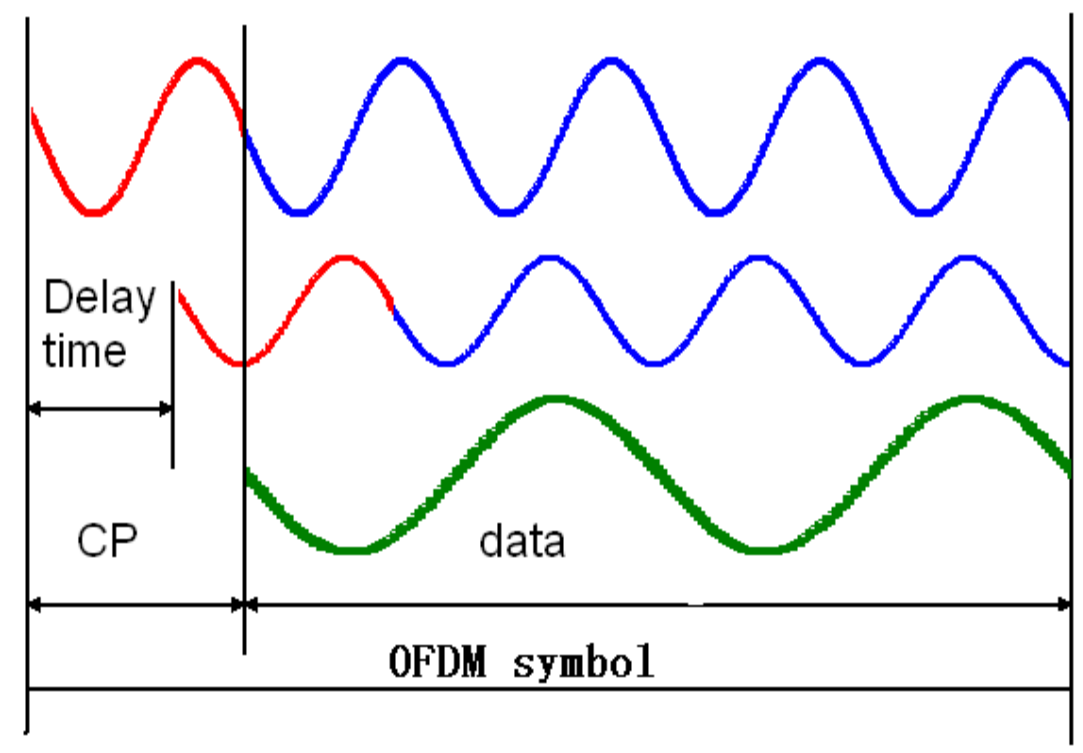

Figure 2.5: Cyclic extension in waveform

Now, after inserting the cyclic extension, as long as the delayed time is shorter than the GI time period, the delayed copies of the OFDM symbol always have the integer numbers of cycles within the FFT interval. In such a case, the ICI and ISI caused by multipath frequency selective fading are totally eliminated.

Choosing the GI time period is also important since a long time comparing to the symbol time can reduce the power efficiency. That's because there is no useful information being sent during this time period. Normally, the GI is chosen to be two to four times of root mean square of the delay spread [10]. 


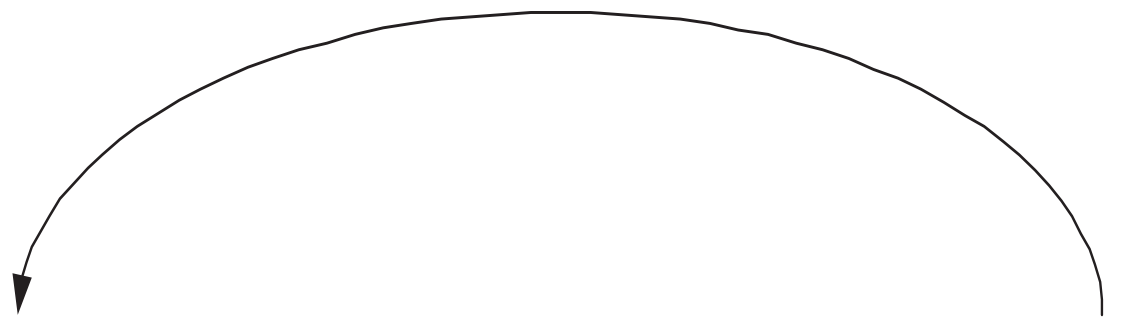

Guard Inter

(Cyclic Exten

Figure 2.6: Cyclic extension in packet

\subsection{Implementation of an OFDM System}

An OFDM transceiver is described in Figure 2.7.From Figure 2.7, it can be seen how the OFDM transceiver works. First, the input data is coded by a certain coding scheme. For example, if the Forward Error Correction (FEC) coding is used, the input bits are binary data that is fed into the coder. Typical FEC codes, such as a block code, convolutional code and Turbo code are used to improve the performance of the OFDM system.

The signal mapping in the block diagram is used to map the bits to complex symbols. Most common signal mapping method are PSK and QAM. The symbols after signal mapping are denoted as $s(k), k=0,1 \ldots N-1$.

In the OFDM design, the serial to parallel $(\mathrm{S} / \mathrm{P})$ converter is considered to realize the concept of parallel data transmission. A S/P block arranges the complex symbols into blocks of $\mathrm{N}$ symbols, where $\mathrm{N}$ is the number of subcarriers in one OFDM block.

The key component of the OFDM modulation is the Inverse FFT (IFFT) transform which is used to modulate the symbols after signal mapping to different subcarriers. After the modulation, the samples of the OFDM signal can be expressed as: 

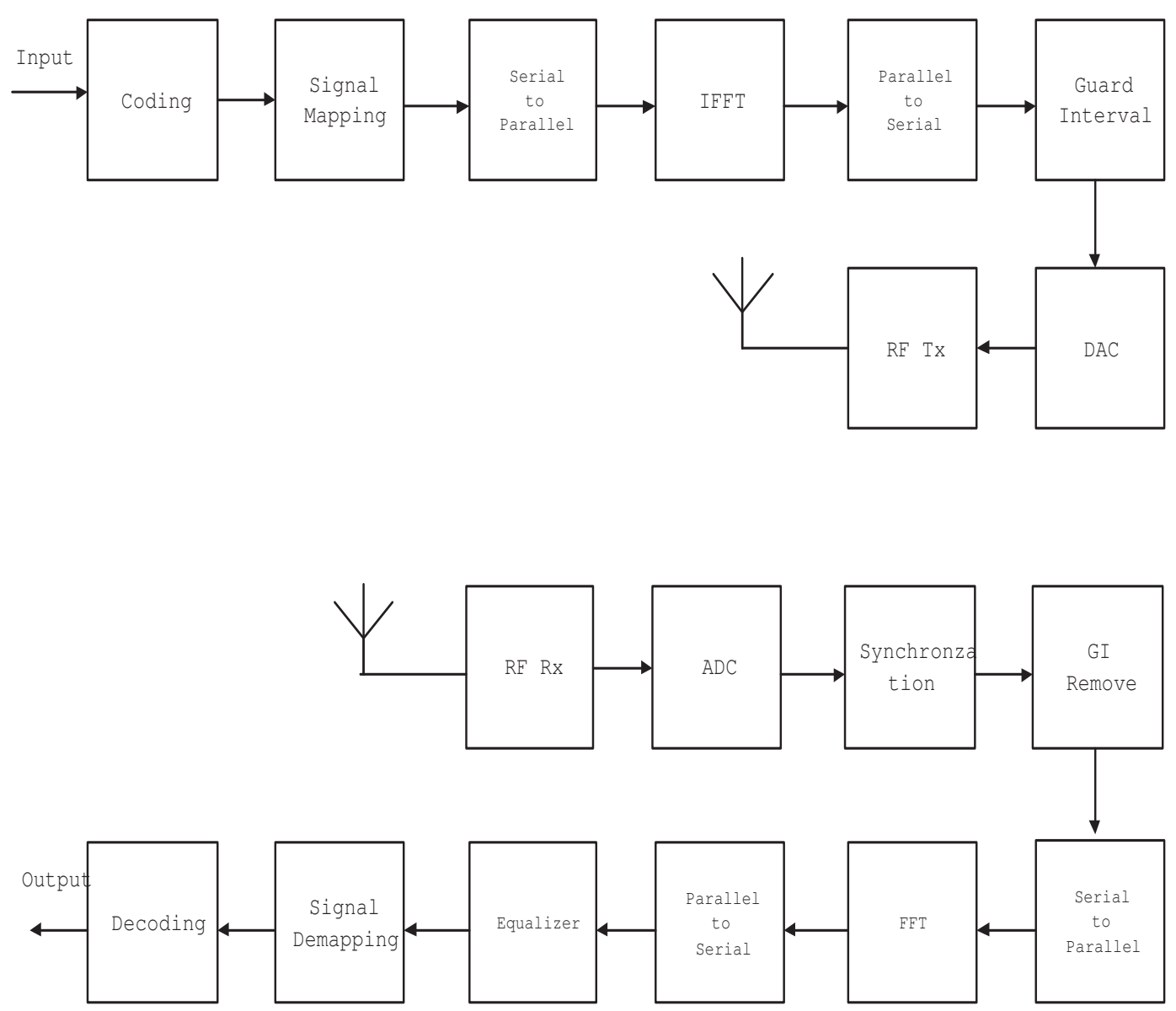

Figure 2.7: Block diagram of the OFDM transceiver

$$
x(n)=\frac{1}{N} \sum_{k=0}^{N-1} s(k) \exp \left(j \frac{2 \pi}{N} k n\right), n=0,1 \ldots N-1
$$

The GI is inserted then to preserve the orthogonality of the subcarriers and to combat ISI. The length of the GI $T_{g}$ is longer than the multipath delay spread, which is denoted as $T_{\text {delay }}$.

A pulse shaping filter with impulse response $g(t)$ is introduced in the DAC. So when the signal passes through the DAC, the complex envelope of the signal can be given:

$$
x(t)=\rho(t) \exp (j \psi(t))=\sum_{m=-\infty}^{\infty} \sum_{n=0}^{N-1} x(n) g\left(t-n T-m N\left(T+T_{g}\right)\right)
$$

where the $T$ is the symbol time and $T_{g}$ is the the length of CP. Finally, the 
signal is amplified and transmitted.

At the receiver, the synchronization is very important since the OFDM system is very sensitive to the synchronization errors. More details are discussed in Section 2.4. Another component of the receiver is the equalizer. Due to the facts that the OFDM system is robust to the ISI and each subchannel is almost flat fading, time domain equalization is not needed. So a simple one tap frequency domain equalizer is applied to equalize the channel.

\subsection{Drawbacks of OFDM}

In the previous sections, the basics of OFDM were briefly introduced. Some drawbacks of OFDM can be concluded due to the nature of its basic theory.

The first important problem of OFDM is the problem of synchronization. In OFDM transmission, the received subcarriers are orthogonal only if the transmitter and receiver are strictly synchronized. Therefore, if there is any frequency offset between them, the ICI can be caused. One related problem is phase noise when the practical oscillator can not only produce carrier at one exact frequency [10]. Both phase noise and frequency offsets introduce the interference and degrade Signal-to-Noise Ratio (SNR). Therefore, the problem of synchronization is a serious problem for OFDM system.

Another important problem for OFDM is the peak power problem. The OFDM signal consists of many independent subcarriers, which can introduce a high PAPR when added up coherently. A high PAPR brings disadvantages for the OFDM system such as increasing the complexity of the DAC and ADC, and decreasing the efficiency of the RF PA which is a essential component of transmitter. In practice, the PA is inherently nonlinear with respect to signals with envelope fluctuations, and a high PAPR often makes the PA working in the nonlinear area. In Chapter 3, more details of the PAPR problem and nonlinear PA will be introduced.

From drawbacks of the OFDM, we could see that RF impairments, such as phase noise and PA nonlinearity, seriously limit the performance of the OFDM. 
That's because the theory design is built up on the assumption of perfect RF devices. These RF impairments should be seriously considered in order to improve the quality of the OFDM system. Therefore, in the next Chapter, the $\mathrm{RF}$ impairments including phase noise, PA nonlinearity, and I/Q imbalance can be introduced in detail.

\subsection{Summary}

OFDM has many advantages which make it an attractive method for the high speed wireless communication system. It is immune to the delay spread of the multipath fading channel, which is very important for improving the quality of wireless communication. The orthogonality of the subcarriers also makes bandwidth usage more efficient. The equalization is also simple since each subchannel is almost flat fading, and the receiver only needs a one-tap frequency domain equalizer to overcome channel effects.

The disadvantages of the OFDM, which already have been introduced in the last Section, are its sensitivity to frequency offsets and phase noise resulting in the non-orthogonality of the subcarriers. Large envelope fluctuations normally expressed in terms of PAPR, can affect the efficiency of the PA.

The block diagram in the Figure 2.7 forms the basic of the simulator in this thesis work. 


\section{Chapter 3}

\section{Dirty RF}

The last Chapter briefly introduces RF impairments, known as "dirty RF", that seriously degrade the performance of the OFDM system. In this Chapter, some of the most important RF impairments which limit the performance of the OFDM system, such as I/Q Imbalance, nonlinearity of power amplifier and phase noise, are presented individually in detail. Theoretical concepts as well as equations are presented to help understanding. Section 3.1 reviews phase noise effects. The I/Q imbalance problem is described in Section 3.2. In Section 3.3, nonlinear effects caused by high power amplifiers are described. In Section 3.4, some other impairments are briefly introduced.

\subsection{Phase Noise Effects}

\subsubsection{Introduction to Phase Noise}

Phase noise should be considered carefully since an accurate cancellation scheme of phase noise can help system designer to relax specifications and maintain a required performance. Phase noise is introduced by the Local Oscillators(LO) at both the transmitter and receiver. Phase noise is a random process caused by the frequency fluctuation of LO. It can be described as two multiplicative distortions. However, for a small phase noise bandwidth, the distortion effect approximately equals to the phase noise effect of sum bandwidth of both processes [11]. Therefore, in this thesis, the focus is limited to the phase noise at the receiver. Figure 3.1 generally shows the effects of the phase noise on the constellation map to describe the notation and noise corruption. 


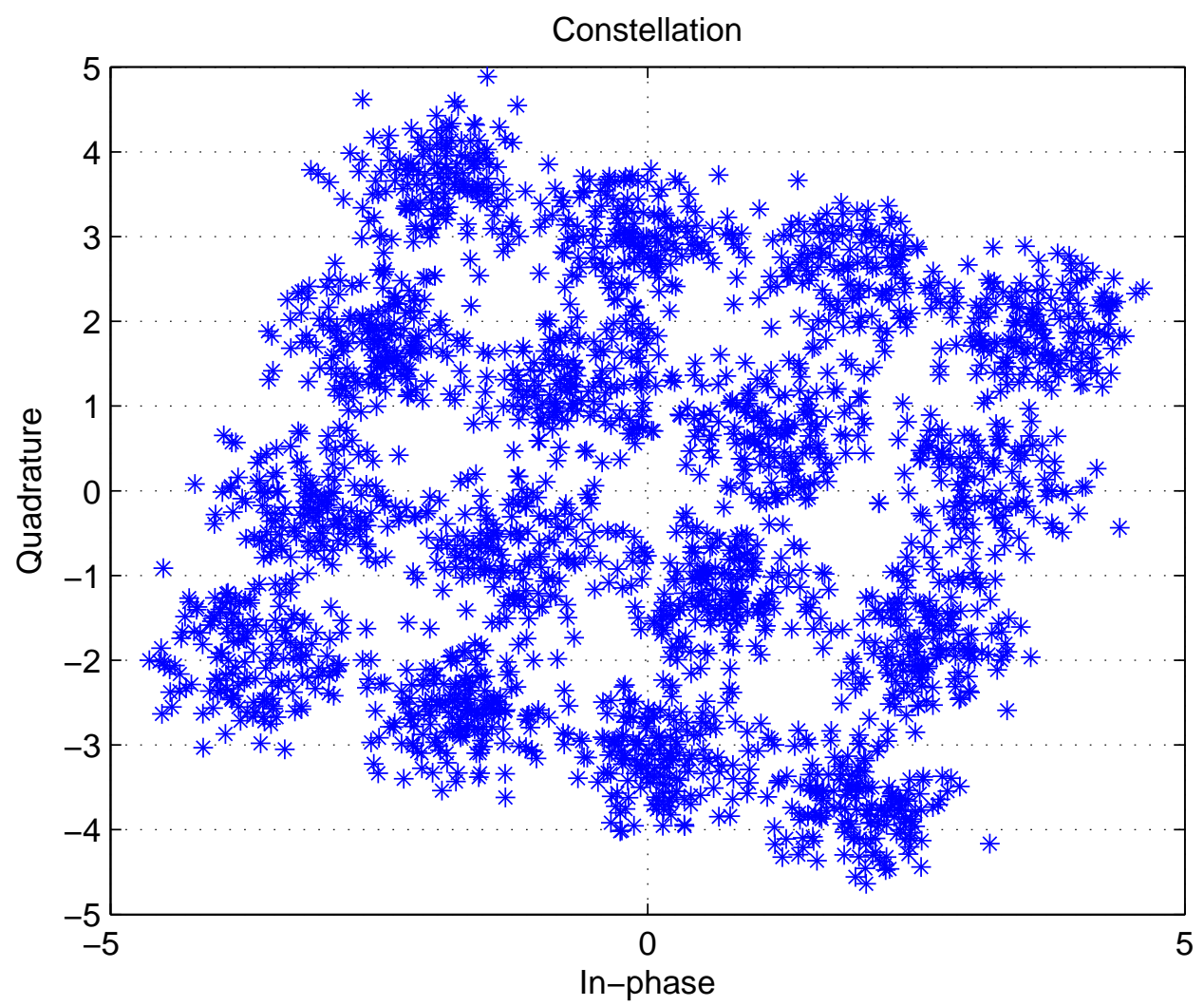

Figure 3.1: Effect of the phase noise on the signal constellation

\subsubsection{Model of Phase Noise}

The discrete-time OFDM symbol with phase noise can be expressed as:

$$
r(n)=(x(n) \otimes h(n)) e^{j \phi(n)}+w(n)
$$

where $x(n), h(n)$ and $w(n)$ denote the samples of the transmitted signal, channel impulse response and the channel noise term respectively, and $\otimes$ denotes convolution. $\phi(n)$ represents the phase noise process at the receiver, and is usually modelled as a Wiener process [12].

After removing the $N_{c p}$ symbols that correspond to the CP and taking the Discrete Fourier Transform (DFT) on the remaining useful $N$ symbols, the demodulated carrier $R_{k}$ is:

$$
R_{k}=\sum_{n=0}^{N-1} r(n) \exp \left(-j \frac{2 \pi}{N} k n\right)
$$


With phase noise, the $R_{k}$ can be expressed as [12]:

$$
R_{k}=X_{k} H_{k} I_{0}+\sum_{l=0, l \neq k}^{N-1} X_{l} H_{l} I_{k-l}+W_{k}, k=-\frac{N}{2} \ldots \frac{N}{2}-1
$$

where $X_{k}, H_{k}$ and $W_{k}$ represent the transmitted symbol on the $k t h$ carrier, sampled channel transfer function and frequency domain noise. The term $I_{k}$ is:

$$
I_{k}=\frac{1}{N} \sum_{n=0}^{N-1} e^{j \phi(n)} e^{\left(-j \frac{2 \pi}{N} k n\right)}
$$

The term $I_{0}$ in the first term of Eq. (3.3), which stems from the phase noise, and does not depend on the subcarrier index, is referred to as Common Phase Error (CPE) [12]. In the OFDM symbol, for a small phase noise, CPE is [12], [13]:

$$
I_{0} \approx e^{j \Phi}=1+j \Phi
$$

where the angle $\Phi$ results from the average of phase noise samples over the symbol period:

$$
\Phi=\frac{1}{N} \sum_{n=0}^{N-1} \phi(n)
$$

It can be seen from Eq. (3.5), CPE results from the complex numbers $e^{j \phi(n)}$. Therefore, it can be viewed as a rotation on the signal constellation. Since it is a constant for all the subcarriers, it can be corrected by a phase rotation. Figure 3.2 shows the phase noise effects on the signal constellation when the effect of CPE dominates.

The second term of the Eq. (3.3) is ICI, corresponding to the summation of the subcarriers each multiplied by a complex number. The spectral component of phase noise in this error term is randomized, therefore, it can not be corrected totally [13]. Figure 3.3 shows the phase noise effects on the signal constellation when the effects of ICI dominates over the effects of CPE. 


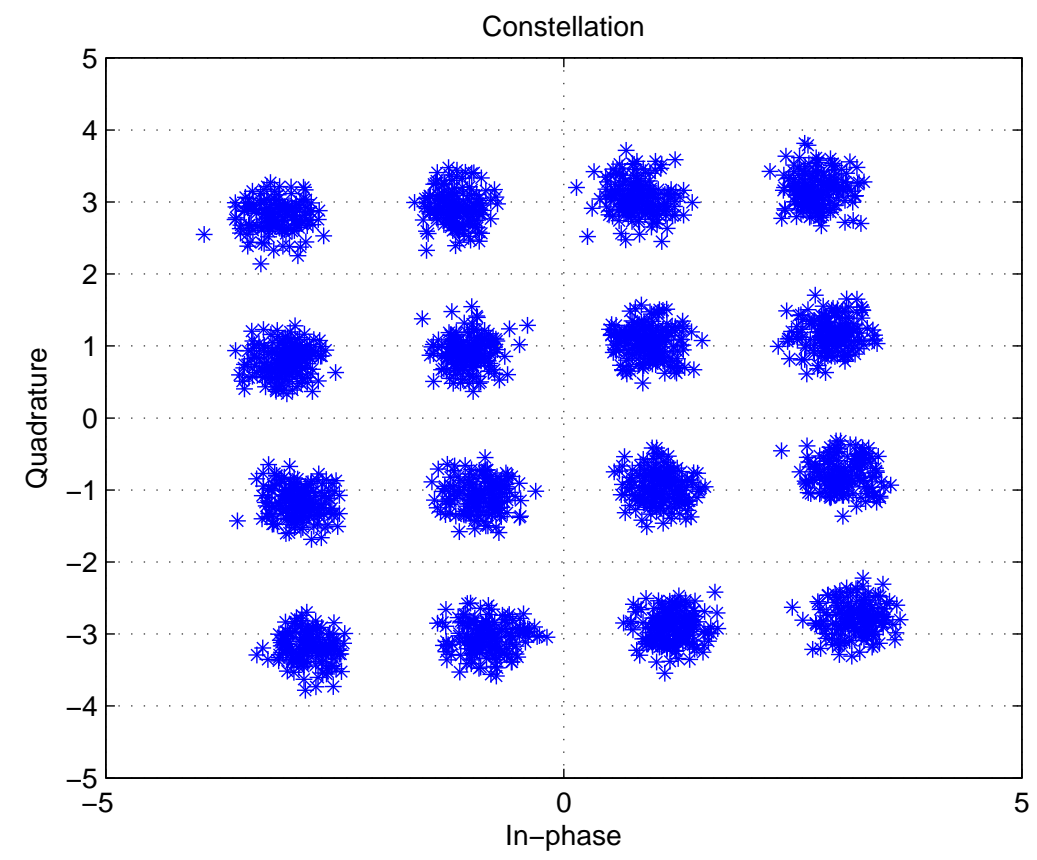

Figure 3.2: Phase noise effects on constellation, CPE dominates

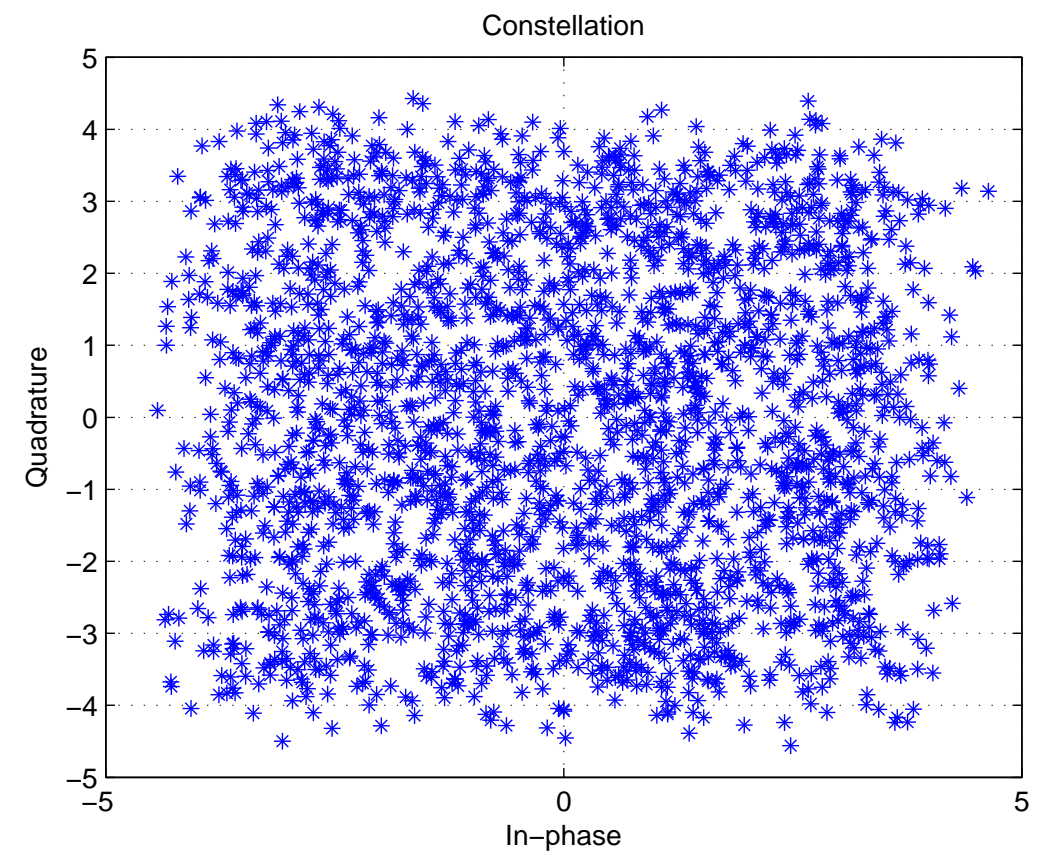

Figure 3.3: Phase noise effects on constellation, ICI dominates 


\subsubsection{Compensation for Phase Noise Effects}

The effects of Phase noise on OFDM system have been analyzed over a period. Several compensation methods based on estimation and correction have been introduced i.e. in [11], [13] - [15].

In [11], an ICI suppression algorithm is presented without considering CPE. The proposed algorithm provides estimation of as many spectral components $I_{k}$ as possible. Then the estimates $\hat{I}_{k}$ are used to do the ICI cancellation.

[13] and [14] focus only on the correction of CPE. [13] takes advantage of the pilot-based correction mechanism and [14] uses the idea of the Kalman filter estimation algorithm. The method in [14] is based on the CPE estimation and does the correction for CPE by derotating all the subcarriers.

In [15], both CPE and ICI have been considered for phase noise correction. A method on the basis of the minimum mean squire estimation (MMSE) algorithm is proposed.

\subsection{I/Q Imbalance Effects}

\subsubsection{Introduction to I/Q Imbalance}

Another major source of impairments in wireless communication system is a mismatch between the I and Q branches or, equivalently between the real and imaginary parts of the complex signal at both the transmitter(during upconversion) and receiver(during down-conversion) [16].

The degradation caused by the $\mathrm{I} / \mathrm{Q}$ imbalance on the performance of the OFDM system has been investigated, for example, in [17], [18]. It can be seen that the I/Q imbalance, which results in ICI [17], should be seriously considered. In this section we present a conventional model of I/Q imbalance at both the transmitter and receiver as well as some compensation methods. 


\subsubsection{Model of I/Q Imbalance in OFDM}

In the up and down-conversion of the transceiver, the incoming signal in the I-path is up-converted or down-converted by the local oscillator at the carrier frequency, while the Q-path signal is up-converted or down-converted with the $90^{\circ}$ phase shift. In reality, matching between the I and Q branches is not perfect, which results in the so called I/Q imbalance.
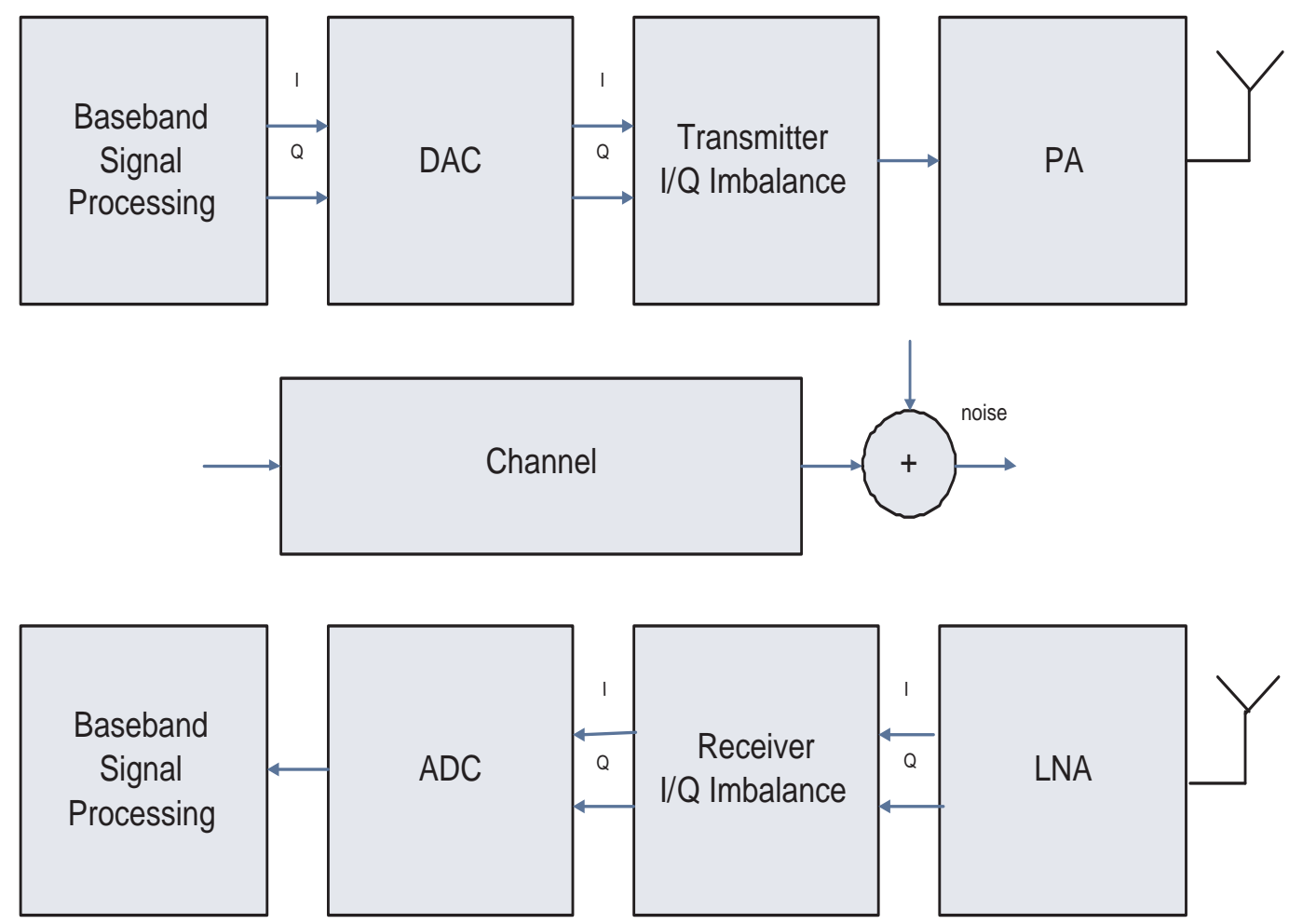

Figure 3.4: OFDM transeiver with I/Q imbalance at transmitter and receiver

A model of the OFDM transceiver with I/Q imbalance is shown in Figure 3.4. First, I/Q imbalance is modelled at the transmitter. Since I/Q imbalance is any mismatch between the I and Q branches from the ideal case, the distorted signal in the time domain can be modelled as [16], [18]:

$$
x_{d}(t)=\mu_{t} x(t)+\nu_{t} x^{*}(t)
$$

The notation $*$ denotes the complex conjugate and $\mu_{t}$ and $\nu_{t}$ are related to the amplitude imbalance $\gamma_{t}$ and phase imbalance $\Delta \varphi_{t}$ between the I and $\mathrm{Q}$ branches at the transmitter. $\mu_{t}$ and $\nu_{t}$ can be expressed as:

$$
\mu_{t}=\cos \left(\triangle \varphi_{t}\right)+j \gamma_{t} \sin \left(\triangle \varphi_{t}\right)
$$




$$
\nu_{t}=\gamma_{t} \cos \left(\triangle \varphi_{t}\right)+j \sin \left(\triangle \varphi_{t}\right)
$$

From the equations it can be seen that if $\gamma_{t}=\Delta \varphi_{t}=0$, then, $\mu_{t}=1, \nu_{t}=0$ and $x_{d}(t)=x(t)$, i.e., there is no $\mathrm{I} / \mathrm{Q}$ imbalance.

Let $r(t)$ represents the received complex signal before being distorted by the receiver I/Q imbalance. Using the same model as at the transmitter, the distorted signal can be modelled as [16] [18]:

$$
r_{d}(t)=\mu_{r} r(t)+\nu_{r} r^{*}(t)
$$

where $\mu_{r}$ and $\nu_{r}$ are similarly to $\mu_{t}$ and $\nu_{t}$, and the $r_{d}(t)$ is the distorted signal after I/Q imbalance at the receiver.

Next, channel effects are considered. Let $h(t)$ be the continuous channel impulse response and $H$ is its frequency response, i.e. $H=F T(h)$. The length of $h$ is assumed to be shorter than the length of OFDM CP. The received signal $r(t)$ can be expressed as:

$$
r(t)=h(t) \otimes x_{d}(t)+w(t)=h \otimes\left(\mu_{t} x(t)+\nu_{t} x^{*}(t)\right)+w(t)
$$

where $w(t)$ is channel noise. If the frequency domain signal vector is denoted as $X=F T(x(t))$, the frequency domain received signal $R$ can be expressed as:

$$
R=\mu_{t} X H+\nu_{t} X_{m}^{*} H+W
$$

Here, ()$_{m}$ denotes the mirroring operation in which the vector indices are reversed [18], i.e. $X_{m}(l)=X\left(l_{m}\right)$, where

$$
l_{m}= \begin{cases}2+N-l, & l=2, \ldots, N \\ l, & l=1\end{cases}
$$

$l_{m}$ is the mirror carrier of subcarrier $l$.

Therefore, when the impacts of the transmitter and receiver I/Q imbalance are considered together with channel effects, Eq. (3.10) can be expressed as: 


$$
r_{d}(t)=\mu_{r} r(t)+\nu_{r} r^{*}(t)=\mu_{r}\left(h \otimes x_{d}(t)+w(t)\right)+\nu_{r}\left(h \otimes x_{d}(t)+w(t)\right)^{*}
$$

$R_{d}=\mu_{r} R+\nu_{r} R^{*}=\left(\mu_{t} \mu_{r} H+\nu_{t}^{*} \nu_{r} H_{m}^{*}\right) X+\left(\mu_{r} \nu_{t} H+\mu_{t}^{*} \nu_{r} H_{m}^{*}\right) X_{m}^{*}+\mu_{r} W+\nu_{r} W_{m}^{*}$

From Eq. (3.12), (3.14) and (3.15), it can be seen that I/Q imbalance causes ICI at the receiver. Since OFDM system is sensitive to ICI, I/Q imbalance may cause severe performance degradation.

\subsubsection{Compensation for I/Q Imbalance Effects}

[16], [18]- [20] introduce several different compensation methods of the I/Q imbalance. [19] focuses on the case where the $\mathrm{I} / \mathrm{Q}$ imbalance is only observed at the OFDM transmitter. A MMSE estimation algorithm is proposed to estimate the $\mu_{t}$ and $\nu_{t}$, then the compensation method is based on the estimated value of $\mu_{t}$ and $\nu_{t}$.

[20] presents several different compensation schemes for I/Q imbalance in the OFDM receiver, including Least Squares (LS) channel estimation and equalization, Least Mean Squares (LMS) equalization and distortion estimation.

Compensation schemes which are presented in [16] and [18] consider both transmitter and receiver I/Q imbalance. [16] proposes two different approaches. In the first scheme, the imbalance is jointly compensated at the receiver after the FFT operation by using an adaptive compensation algorithm. The second approach performs pre-distortion after the IFFT operation at the transmitter, which makes the issue become to estimate $\mu_{t}$ and $\nu_{t}$ that have been introduced in Eq. (3.9). Then, compensation for the I/Q imbalance is accomplished based on the estimate before the FFT operation at the receiver. [18] introduces an OFDM system with four-tap adaptive equalizer at the receiver to do the compensation. This scheme is also based on the LMS algorithm. 


\subsection{Nonlinear Power Amplifier}

\subsubsection{Characteristics of Nonlinear Power Amplifier}

The PA is an essential component of the transmitter in a modern wireless communication system. It serves to generate the required transmit power needed for overcoming transmission loss between the transmitter and receiver. Ideally, the PA should be linear. However, in reality all the PAs have nonlinear input-output characteristics which generates nonlinear distortion with respect to the signals with envelope fluctuations [21].

In this section, the amplifier characteristics are described at the system level and the nonlinearity is considered as memoryless, which means that the output signal of the PA only depends on the current input signal. The PA is inherently amplitude dependent which means that the distortion is a function of the amplitude of the input signal. A memoryless PA model is characterized in terms of amplitude-to-amplitude distortions (AM-AM) and amplitude-tophase distortions (AM-PM).

At first, the characteristic and definition of the PA operation point are shown in Figure 3.5. Figure 3.5 shows that when the input power remains at a low level, the output power of the PA is approximately linear with respect to the input power. As the input power increases, a PA AM/AM curve becomes more nonlinear. Finally when the input power does not drive the change of the output power, the amplifier reaches its saturation point.

Some parameters are used to describe how efficiently the amplifier is used. Input Back-off (IBO) is the difference in $\mathrm{dB}$ between the input saturation power and input average power, which is expressed as:

$$
I B O=10 \log _{10}\left(\frac{\text { Pin }_{\text {sat }}}{\text { Pin }_{\text {ave }}}\right)
$$

Output Back-off (OBO) is the difference between the output saturation power and output average power, which is defined as:

$$
O B O=10 \log _{10}\left(\frac{\text { Pout }_{\text {sat }}}{\text { Pout }_{\text {ave }}}\right)
$$




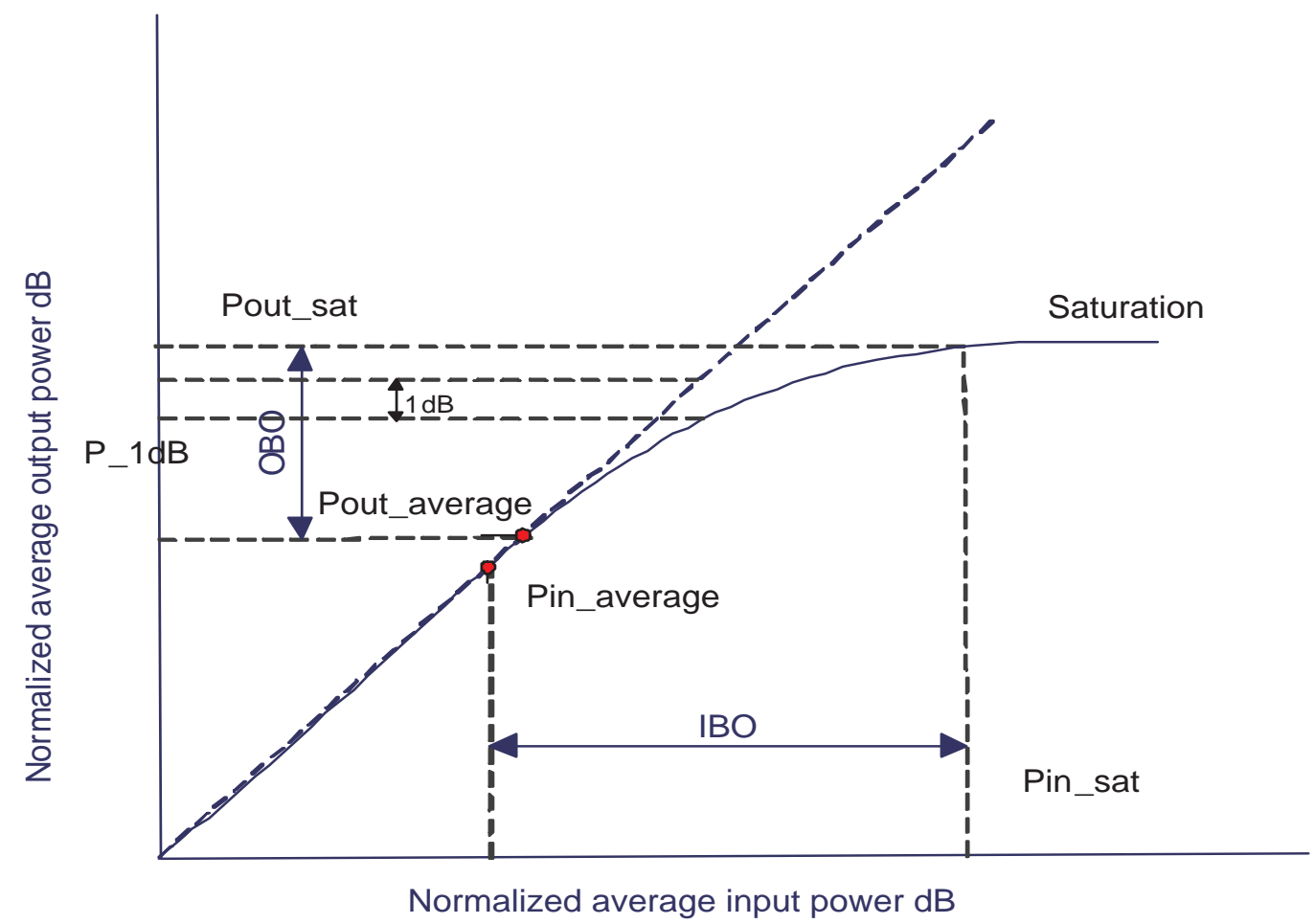

Figure 3.5: Input/Output curve of PA

where Pin sat and Pout sat stand for the input and output saturation power respectively.

Another frequently used parameter for the amplifier is $1 \mathrm{~dB}$ compression point, which gives the value of the output power when the difference between the true output power and ideal uncompressed power is $1 \mathrm{~dB}$.

The output-input relation of the memoryless nonlinear PA can be described as [21]:

$$
u(t)=F(x(t))
$$

where $x(t)$ is the output of the DAC, and $F()$ is a nonlinear function. From Eq. (2.2), the output of the nonlinear power amplifier can be written as:

$$
u(t)=F_{A}(\rho(t)) e^{j F_{P}(\rho(t))} e^{j \psi(t)}
$$


where $F_{A}()$ and $F_{P}()$ are the AM-AM and AM-PM conversion functions of the amplifier.

\subsubsection{PAPR Problem in OFDM}

One reason why the nonlinearity of PA should be considered seriously is that the large peak power of the OFDM signal sometimes makes the PA inefficient. As mentioned in Chapter 2, when adding up subcarriers with the same phases, the peak power is $N$ times than the average power of the signal on each subcarrier. This results in a high Peak-to-Average Power Ratio (PAPR). Such high PAPR problem associated with multicarrier signals is one of the principal drawbacks of OFDM. A high PAPR makes the PA work with large IBOs, resulting in inefficient use of the amplifier. High PAPR also increases the complexity of the ADC and DAC [21].

The PAPR is defined as:

$$
P A P R=\frac{\max _{0 \leq t \leq T}|x(t)|^{2}}{E[|x(t)|]^{2}}
$$

where $\max _{0 \leq t \leq T}|x(t)|^{2}$ is the maximum power of the signal and $E[|x(t)|]^{2}$ is the average power. Another factor used is the Crest Factor $(\mathrm{CF})$ which is defined as the square root of PAPR:

$$
C F=\frac{\max _{0 \leq t \leq T}|x(t)|}{E[|x(t)|]}
$$

From Eq. (3.20), it can be seen that the high PAPR can be reduced either by reducing the maximum signal power or by increasing the average power. In reality, reducing the maximum signal power is used in most cases because increasing the average power causes more interference. Several approaches have been proposed to reduce the high PAPR and they can be divided into three main categories [10]. The first approach relies on coding technique which uses a special coding, i.e. Forward Error Correct (FEC), to reduce the large PAPR. This approach does not cause interference but it increases the complexity of the transmitter and decreases the transmission rate. The second approach is the signal distortion technique, which simply reduces the signal amplitude by 
distorting the signal. Clipping technique and peak windowing, for instance, belong to this approach. The last one is based on scrambling each OFDM symbol with different scrambling sequences and then selecting the sequence that gives the smallest PAPR.

Although there are many techniques for reducing high PAPR, all these approaches have some corresponding disadvantages, such as signal distortion and complexity of the implementation. These approaches also cannot guarantee that the signal after processing can avoid PA nonlinear distortion.

\subsubsection{Frequently Used Models of Nonlinear Power Am- plifier}

In this Section, we review some frequently used models of memoryless nonlinear power amplifiers.

\section{Soft Envelope Limiter}

The Soft Envelope Limiter (SEL) is a model of the ideal power amplifier. The AM-AM conversion of the SEL is linear with respect to the input signal before it meets the saturation point and SEL does not affect the phase of the signal. The AM-AM and AM-PM of SEL functions are [22]

$$
\begin{gathered}
F_{A}(\rho)= \begin{cases}\rho, & \rho \leq A_{\text {sat }} \\
A_{\text {sat }}, & \rho>A_{\text {sat }}\end{cases} \\
F_{P}(\rho)=0
\end{gathered}
$$

where $\rho$ is input amplitude. Figure 3.6 shows the AM-AM function of a SEL when $A_{\text {sat }}=1$.

\section{Solid-State Power Amplifier}

A Solid-State Power Amplifier (SSPA) is described by Rapp's model [23]. The AM-PM conversion of SSPA is small so that it can be neglected. The AM-AM and AM-PM functions of SSPA are as follows: 


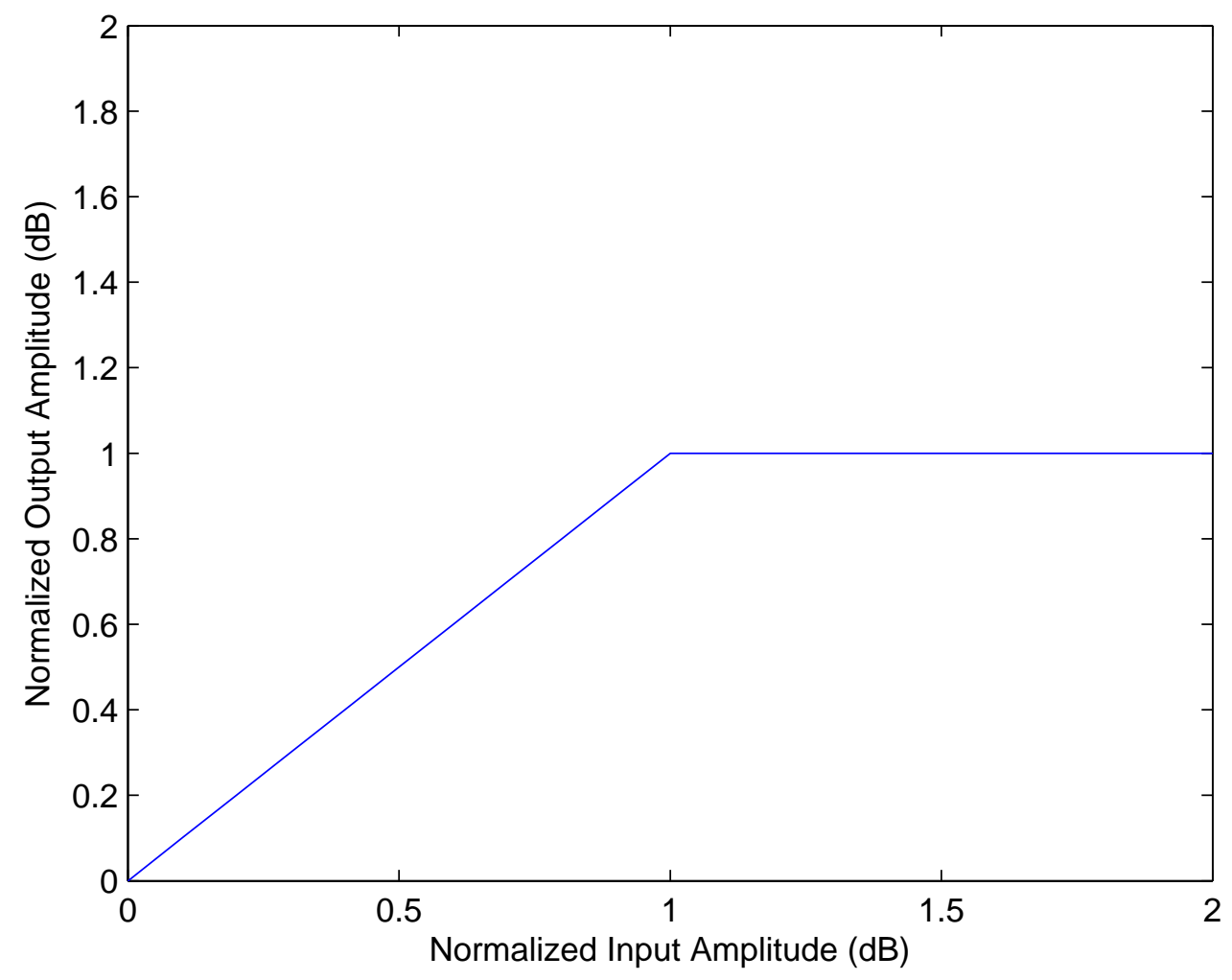

Figure 3.6: AM-AM function of a SEL

$$
\begin{gathered}
F_{A}(\rho)=\frac{\rho}{\left[1+\left(\frac{\rho}{A_{o}}\right)^{2 p}\right]^{\frac{1}{2 p}}} \\
F_{P}(\rho)=0
\end{gathered}
$$

where $p$ is the parameter that controls the smoothness of the function. The figure below shows that as the value of $p$ increases, the linearity of the model becomes better, where $A_{o}=1$.

\section{Travelling-Wave Tube Amplifier}

A Travelling-Wave Tube Amplifier (TWTA) is modelled according to Saleh TWT model [24]. AM-AM and AM-PM conversions of TWTA are as follow

$$
F_{A}(\rho)=A_{\text {sat }}^{2} \frac{\rho}{\rho^{2}+A_{\text {sat }}^{2}}
$$




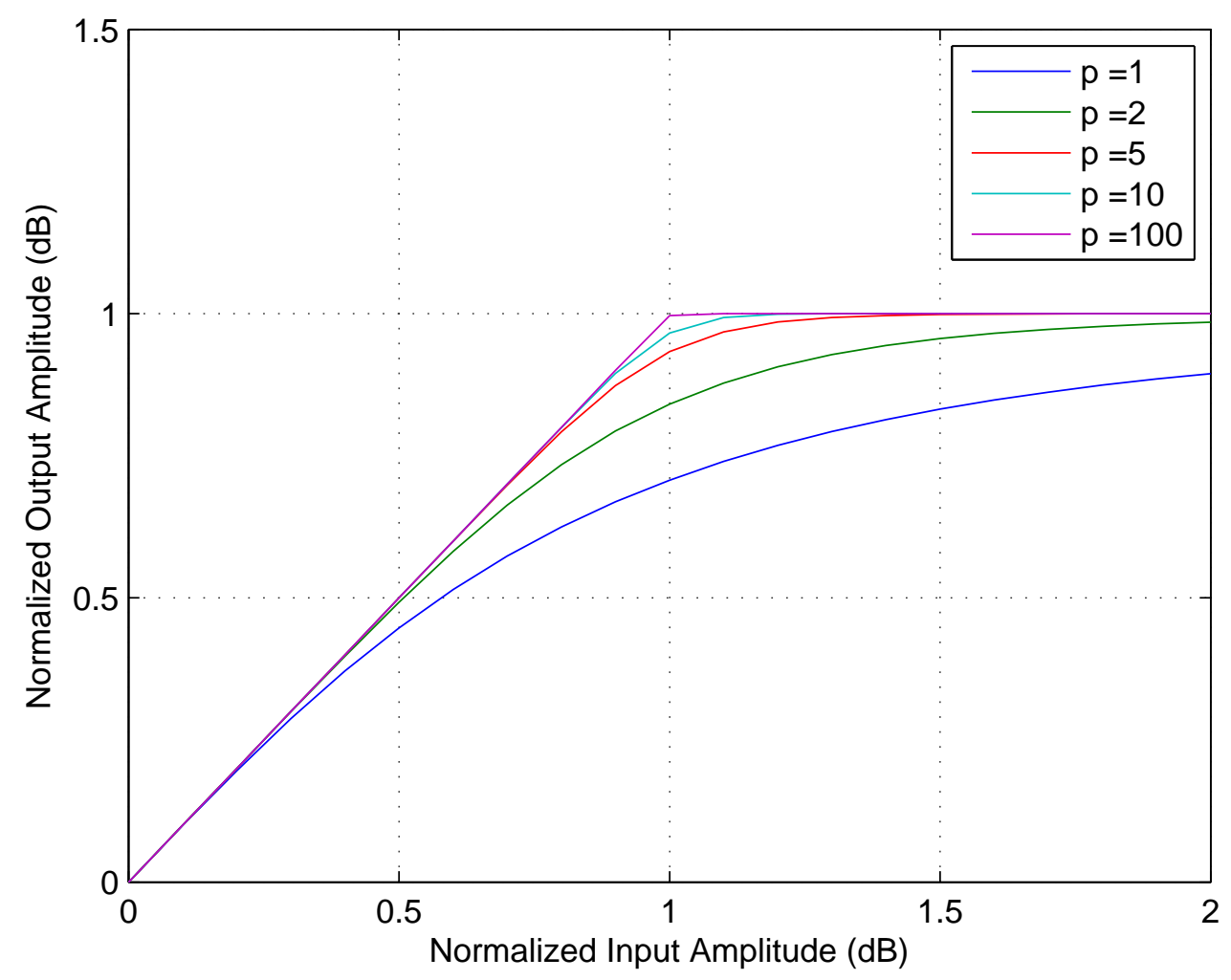

Figure 3.7: AM-AM function of a SSPA with different $p$ values

$$
F_{P}(\rho)=\alpha \frac{\rho^{2}}{\rho^{2}+\beta A_{\text {sat }}^{2}}
$$

where $A_{\text {sat }}$ is the input saturation voltage. $\alpha$ and $\beta$ are shape parameters. From Eq. (3.27), it can be seen that the TWTA has strong AM-PM conversion. Figure 3.7 and 3.8 presents AM-AM and AM-PM curves respectively, where $A_{\text {sat }}=2, \alpha=\frac{\pi}{3}$ and $\beta=1$.

\subsubsection{Effects of Nonlinearity on OFDM Signal}

The effects of the nonlinearity on the OFDM signal can be divided into inband and out-of-band interference effects. In-band interference effects distort the signal spectrum and degrade the system performance. Out-of-band interference effects generate spectral spreading and thus create adjacent channel interference [25]. 


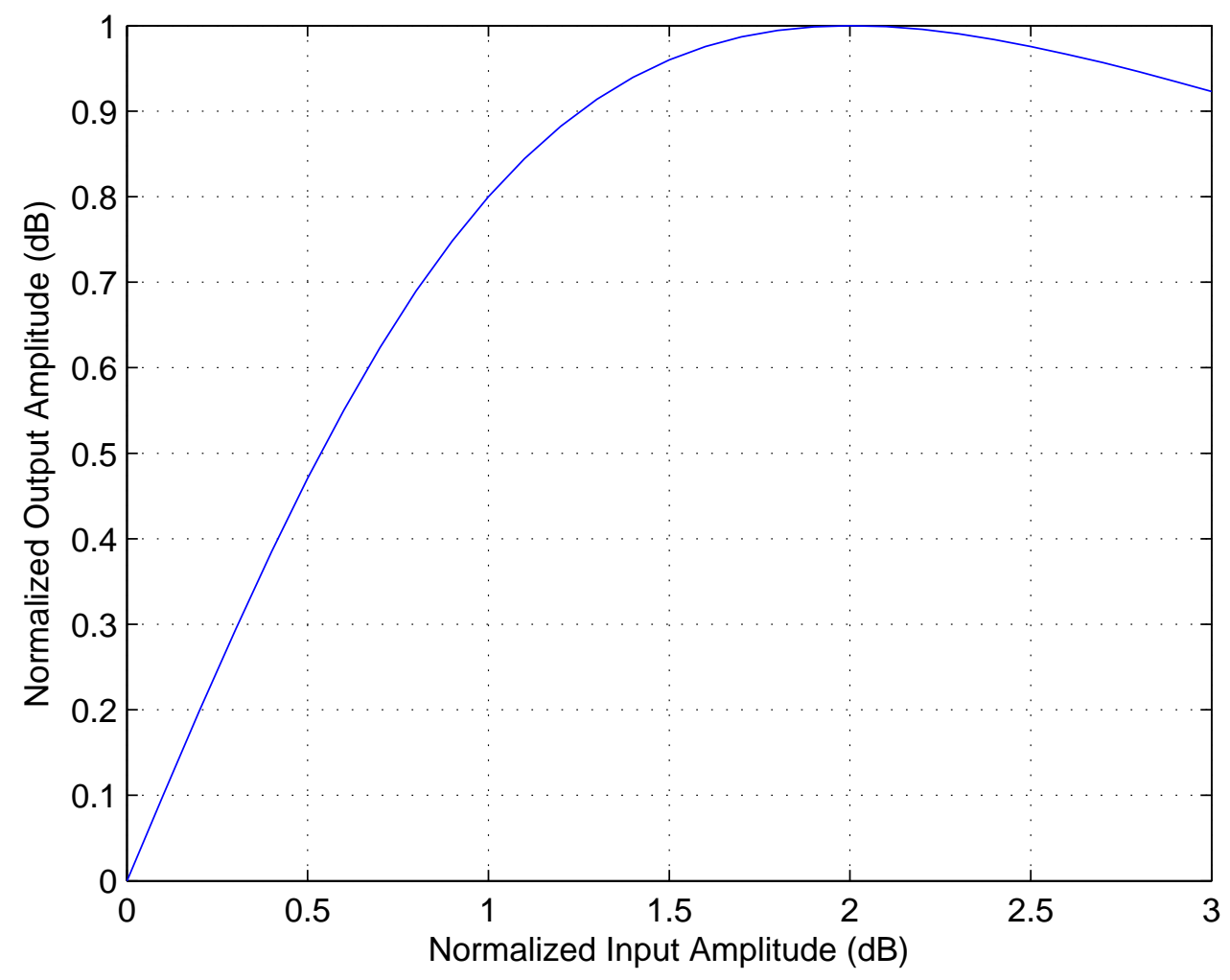

Figure 3.8: AM-AM function of a TWTA

In-band interference effects have been studied extensively. For example, in [22], a theoretical analysis of the nonlinear power amplifier has been shown. An additive noise like effect which is called Nonlinear Distortion (NLD) noise, rotation, and attenuation are considered as the main effects of the in-band interference. Figure 3.10 and 3.11 show the constellation of an OFDM signal after SSPA and TWTA respectively. The parameters of the simulation are 16-QAM, 64 subcarriers.

The output of the nonlinear PA can be expressed as [22]:

$$
u(t)=K(t) x(t)+d(t)
$$

where $K(t)$ is an deterministic factor which defines the attenuation and rotation of signal, $d(t)$ is additive noise with zero mean, variance $\sigma_{d}^{2}$ and it is uncorrelated to the input. $K(t)$ and $d(t)$ depend on the AM-AM and AM-PM functions. $K(t)$ can be expressed as [22]: 


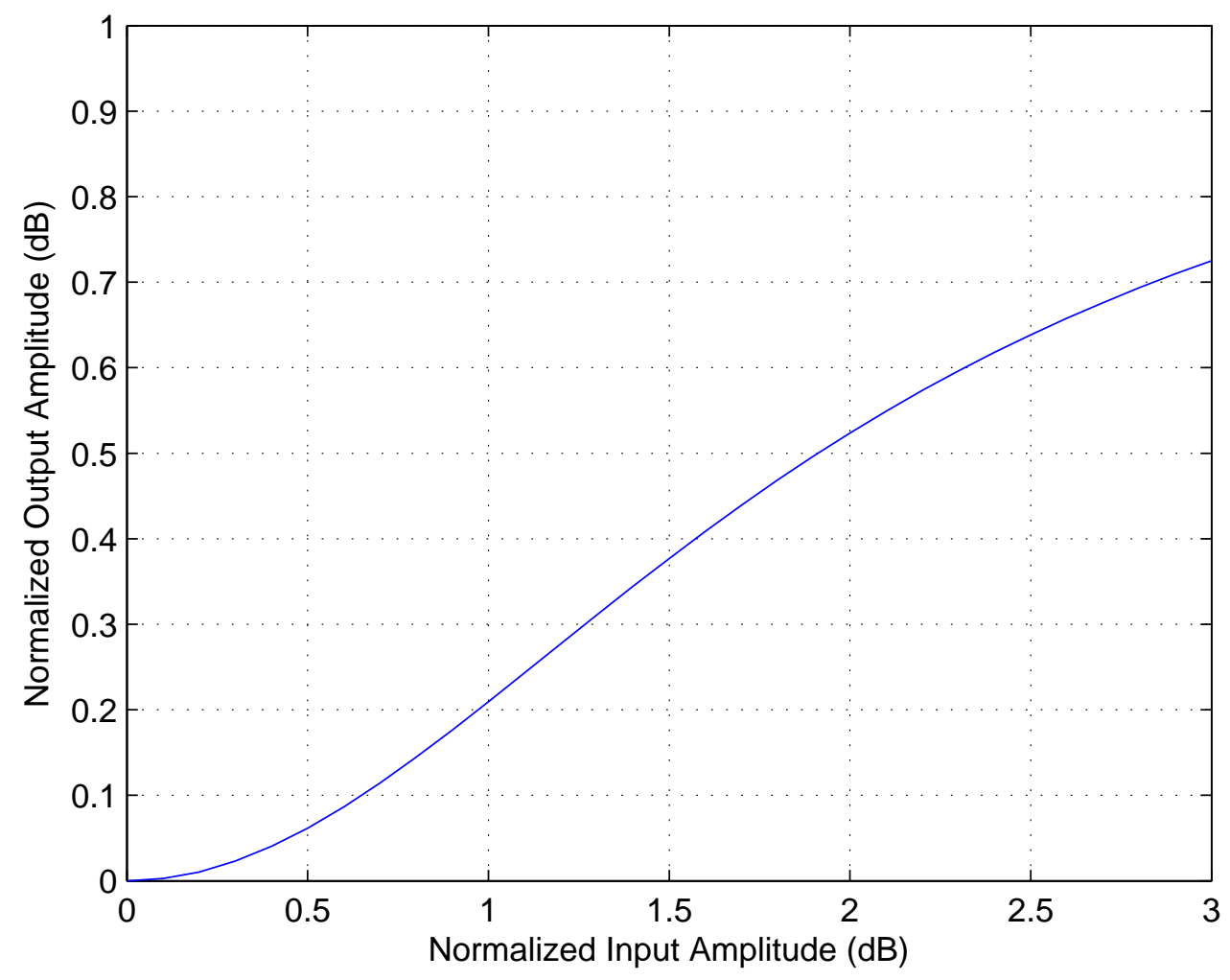

Figure 3.9: AM-PM function of a TWTA

$$
K(t)=\frac{1}{2} E\left[L^{\prime}(\rho)+\frac{L(\rho)}{\rho}\right]
$$

where $L(\rho)=F_{A}(\rho) e^{j} F_{P}(\rho)$. Under some certain conditions which are presented in [22],i.e., mean of input signal is zero and rectangular pulse shaping filter is used. $K(t)$ is independent of $t$,

$$
K(t) \approx K_{o}
$$

In the discrete form, Eq. (3.26) becomes:

$$
u_{m}(n)=K_{o} x_{m}(n)+d_{m}(n)
$$

the variance of $d_{m}(n)$ can be obtained as [22]:

$$
\sigma_{d}^{2}=E\left[|L(\rho)|^{2}\right]-K_{o}^{2} E\left[\rho^{2}\right]
$$




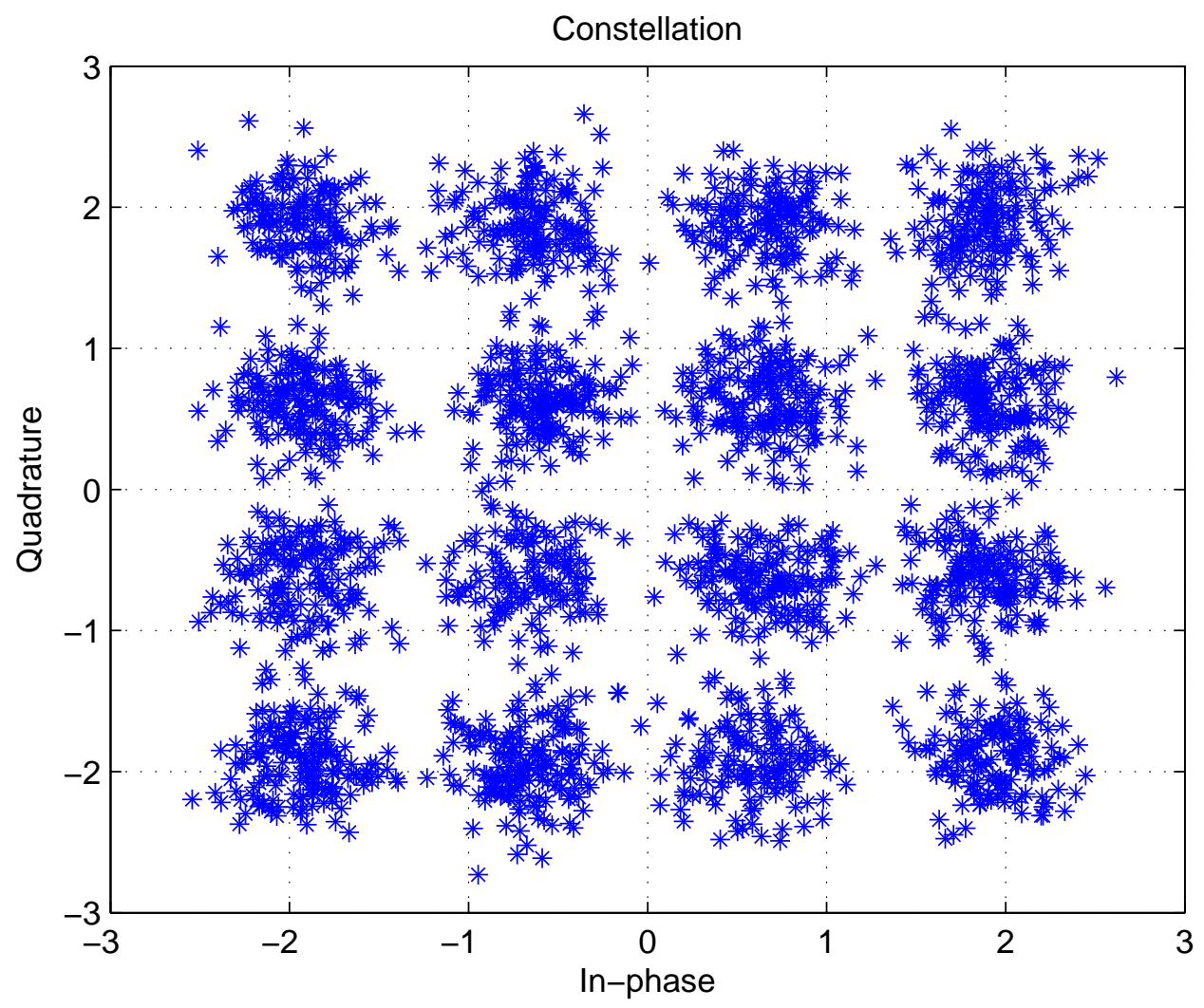

Figure 3.10: Constellation of an amplified OFDM signal after SSPA with OBO $=4.32 \mathrm{~dB}$

If the pulse shaping of the transmitter is rectangular, after the FFT block of the receiver, the NLD noise is the sum of $N$ uncorrelated random variables. It can be assumed as Gaussian according to the central limit theorem [22]. Therefore, the variance of the NLD noise is:

$$
\sigma_{D}^{2}=N \sigma_{d}^{2}
$$

The bit error ratio (BER) can be evaluated as a function of the modulation format and SNR. The SNR of the signal after passing through the channel is:

$$
S N R=\frac{\left|K_{o}\right|^{2} \text { Pin }}{\sigma_{D}^{2}+\sigma_{w}^{2}}
$$

where Pin is the power of the input signal and $\sigma_{w}^{2}$ is channel noise power. Therefore, for M-QAM modulation, the BER function is derived in [22] as: 


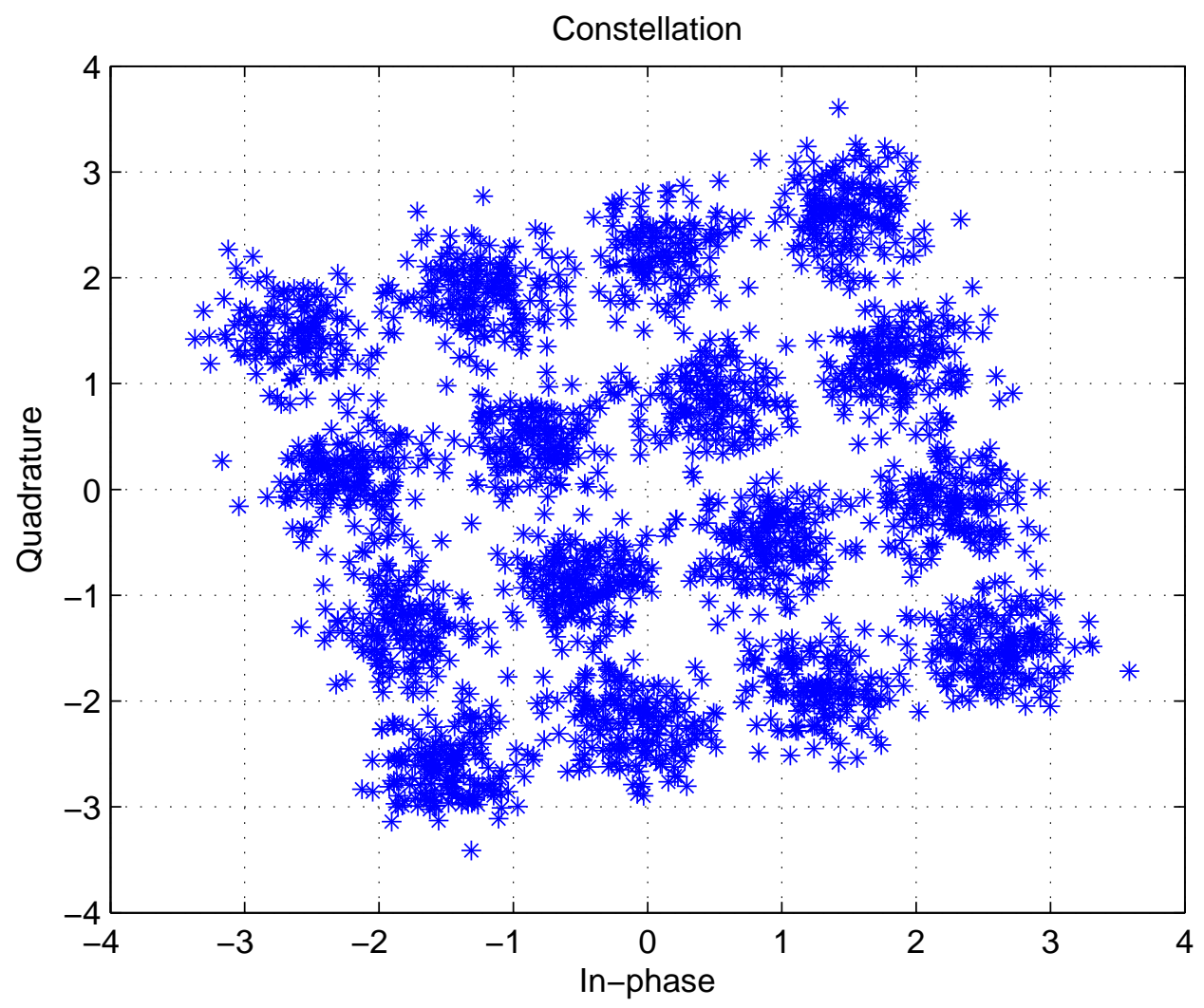

Figure 3.11: Constellation of an amplified OFDM signal after TWTA with $\mathrm{OBO}=3.24 \mathrm{~dB}$

$$
P b=\frac{2}{\log _{2} M} \frac{\sqrt{M}-1}{\sqrt{M}} \operatorname{erfc}(\sqrt{S N R})
$$

The parameter $K_{o}$ and $\sigma_{D}^{2}$ can be analytically calculated, so that the BER can be evaluated in the case of the rectangular pulse shaping. If the impulse response of the pulse shaping filter is non-rectangular, evaluation of $\sigma_{D}^{2}$ is not easy because a NLD noise sample at the input of FFT depends on the whole transmission signal $u(t)$ instead of $u_{m}(n)$ [22].

Another parameter which is used to describe the performance of nonlinear PA is Total Degradation (TD). It is used to describe the performance degradation caused by nonlinear distortion noise and OBO:

$$
T D=S N R_{\text {nonlinear }}-S N R_{\text {linear }}+O B O
$$


The $S N R_{\text {nonlinear }}$ is the required SNR to obtain a fixed BER when the nonlinearity is presented and the $S N R_{\text {linear }}$ is the required SNR to obtain the same BER in absence of nonlinearity. In [26] TD in $\mathrm{dB}$ can be evaluated as:

$$
T D=10 \log _{10}\left(\frac{\frac{\text { Pout }}{\left|K_{o}\right|^{2} \text { Pin }}}{1-\frac{2}{3}(M-1) S N R_{\text {linear }}\left(\frac{\text { Pout }}{\left|K_{o}\right|^{2} \text { Pin }}-1\right)}\right)+O B O
$$

Nonlinear PA distorts the spectrum of an OFDM signal and causes out-ofband interference. These out-of-band interference effects can be analyzed in the frequency domain. The spectrum should be strictly bandlimited if impulse response of phase shaping filter is bandlimited. However, because of the nonlinearity of the PA, the spectrum of a signal would have out-of-bound power and spectrum spread. This can result in out-of-band interference.

From this Section, we can observe that nonlinear distortion obviously has undesirable effects on the OFDM signal. In order to obtain more effective and distortion-free amplification, a number of linearization techniques have been presented. In the next Section, some linearization techniques are reviewed.

\subsubsection{Nonlinear Distortion Cancellation Technique}

There exists a number of linearization techniques which can be divided into a few groups: feedback, feedforward, predistortion and others [27].

\section{Feedback Techniques}

One method for reducing the amplifier distortion is the feedback techniques [27]. Here the basic theory of feedback technique is reviewed. Polar loop feedback and Cartesian feedback are also introduced.

A block diagram which illustrates the feedback technique is given in Figure 3.12. The generalised feedback system contains a feedforward path and a feedback path. The feedforward path contains a nonlinear power amplifier here and the feedback path may contains numbers of different elements [27]. For simplest case, the divider is considered in this thesis. The comparator is to 


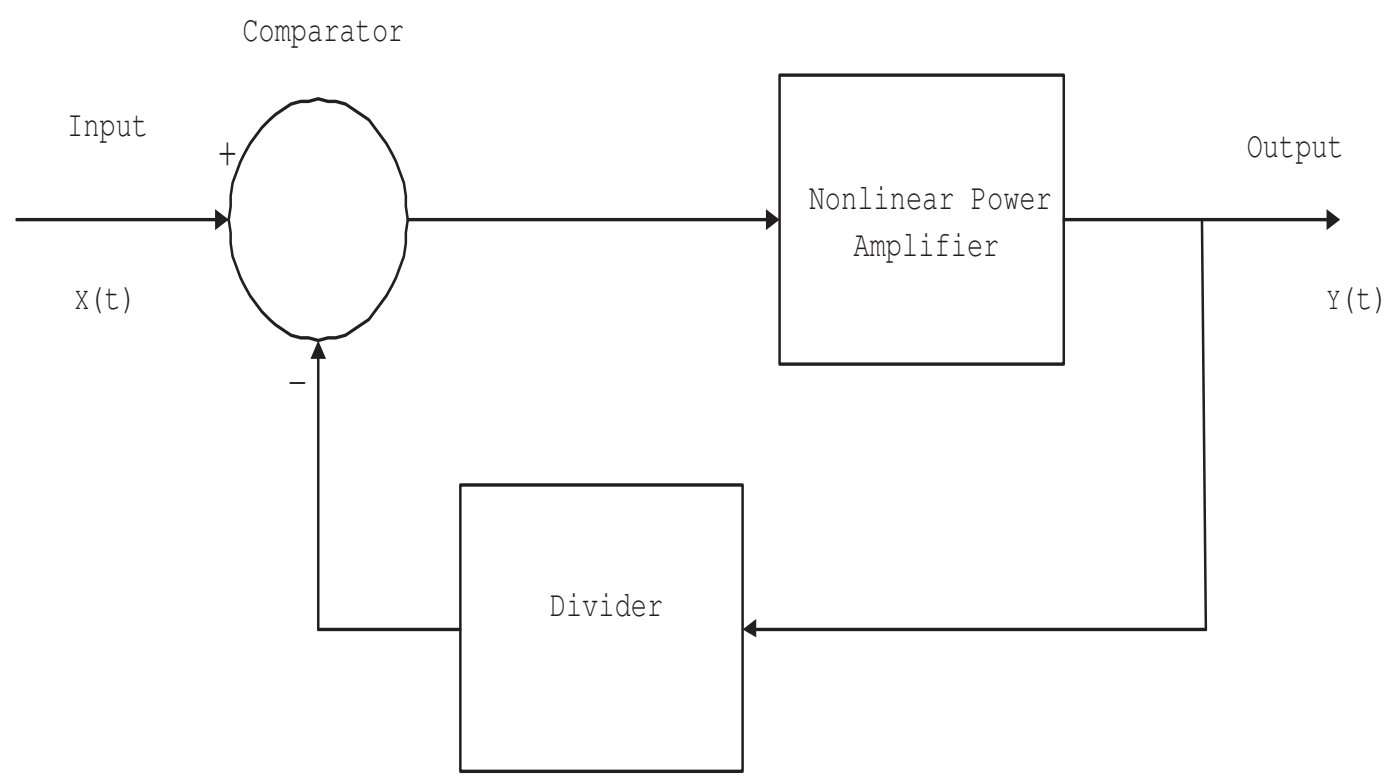

Figure 3.12: Feedback applied around an Amplifier with Distortion

derive an error signal from input signal and feedback output signal.

Polar feedback can be applied to only the amplitude or both the phase and amplitude corrections of the input signal. Phase detection and correction are more complicated to perform than simple amplitude correction [21]. One key issue of the polar feedback is the bandwidth requirement for the error amplifier. In reality, the phase amplifier requires higher bandwidth [21].

Another commonly used scheme is Catersian feedback. A simplified block diagram of Cartesian feedback model is shown in Figure 3.13. The Cartesian feedback model needs a demodulator in the feedback loop which is a source of nonlinear and linear errors as well as noise. Cartesian correction splits the signal into I and Q channels, which is used to keep track of amplitude and phase variations. The Cartesian feedback model is sensitive to the integrity of the circuit. A relative drawback of Cartesian feedback is a narrow bandwidth [27].

\section{Feedforward Techniques}

A block diagram of the typical feedforward system model is presented in Figure 3.14. The basic theory of the feedforward system is concluded as follow. The 


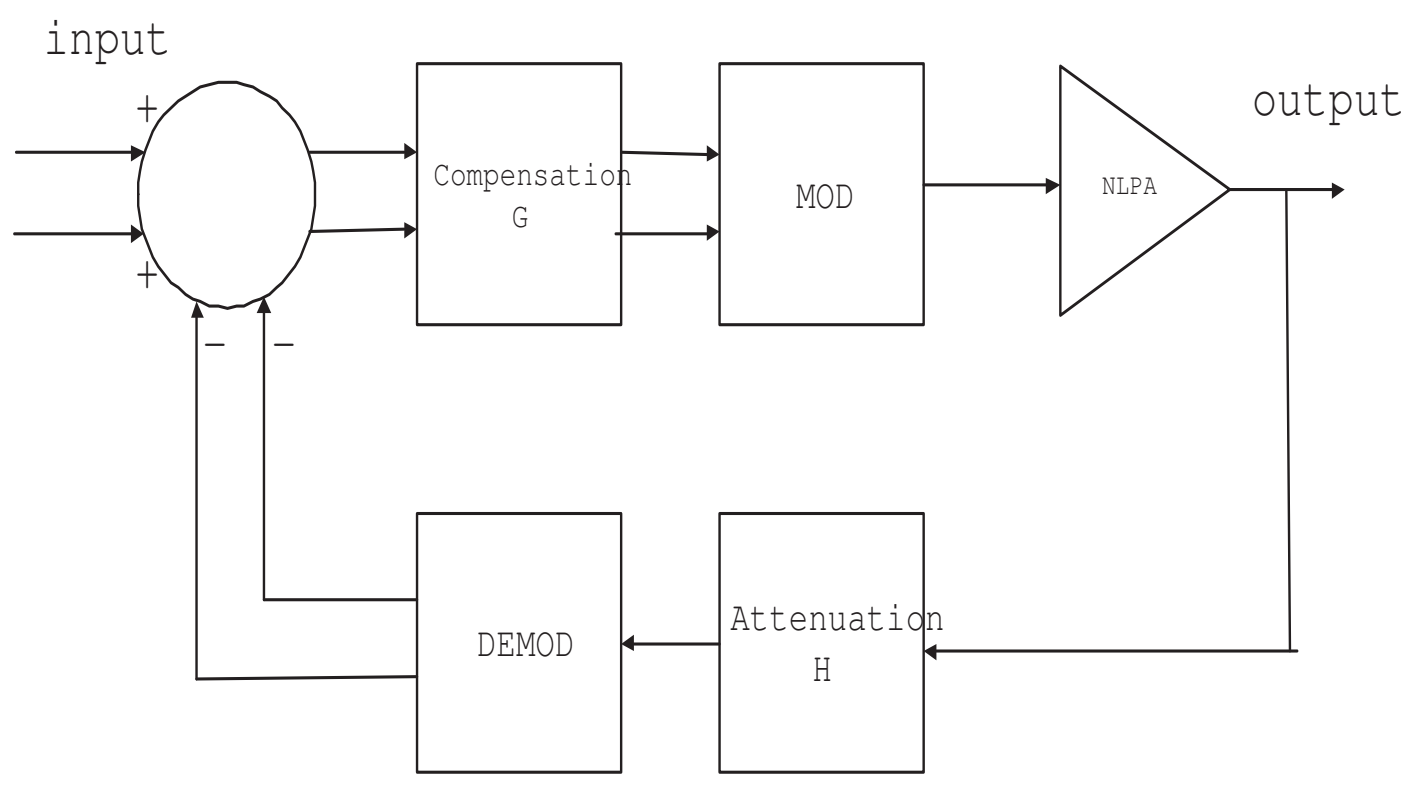

Figure 3.13: Cartesian feedback model

feedforward system has a signal cancellation loop. The distorted amplifier output from the input is subtracted from a delayed sample of an undistorted input signal. If the amplifier has no gain or phase distortion, the result should be zero. If the amplifier has distortion effect, the output of subtractor is the error signal containing distortion products. The error signal then is amplified to its desired level by the error amplifier and recombined with the delayed output of the main amplifier to eliminate the distortion from the main output signal [21].

Although the basic operation of the feedforward system is simple to understand, it certainly has some drawbacks. First of all, it needs an additional PA, so the related problem of the nonlinear contribution of this PA should be considered carefully. The second problem is that the feedforward system requires precise amplitude and phase control. More details about the feedforward techniques are well explained in [27].

\section{Predistortion Techniques}

The predistortion technique is conceptually a simple form for linearization of the nonlinear PA [27]. Different predistortion techniques are introduced in [21] , [27]- [30]. 


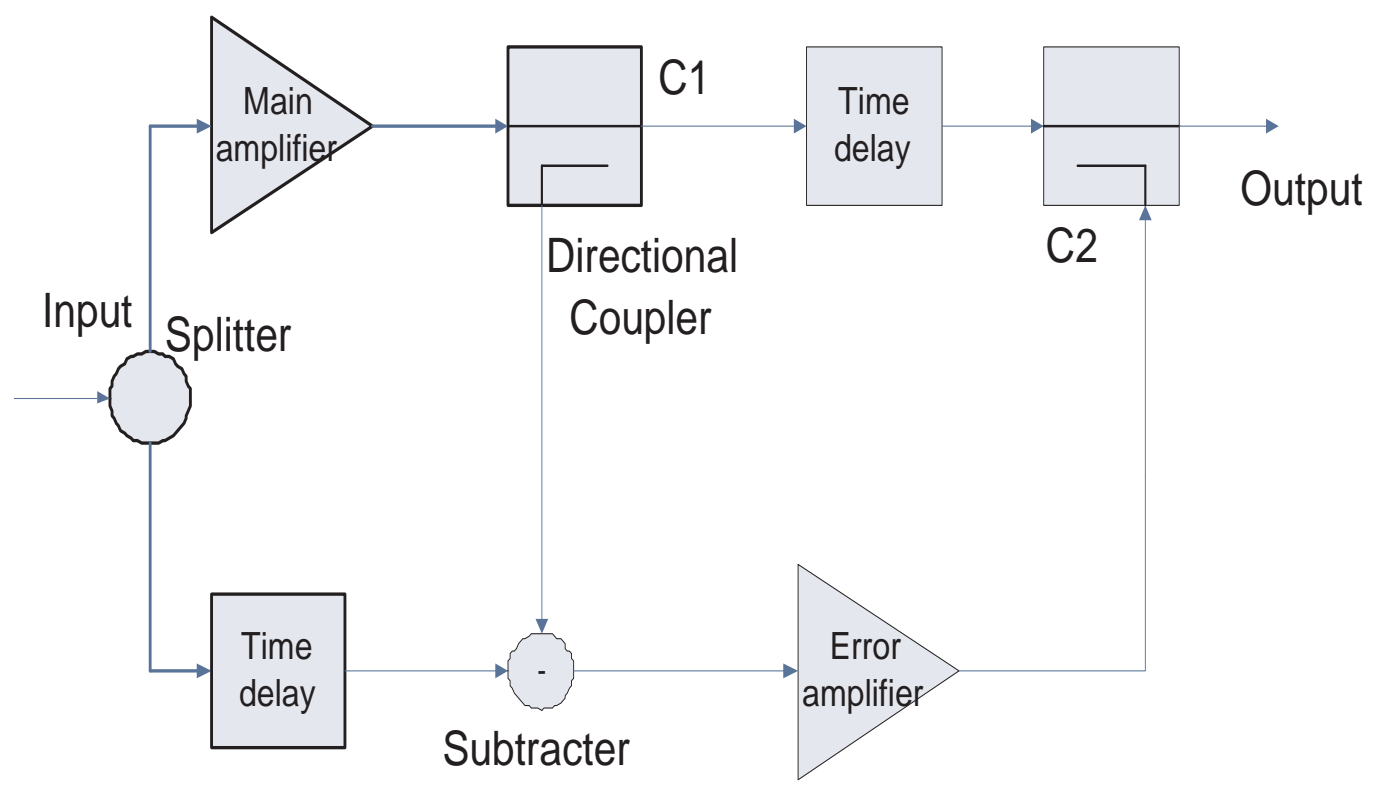

Figure 3.14: Feedforward system model

The basic form of the predistortion scheme is shown in Figure 3.15.

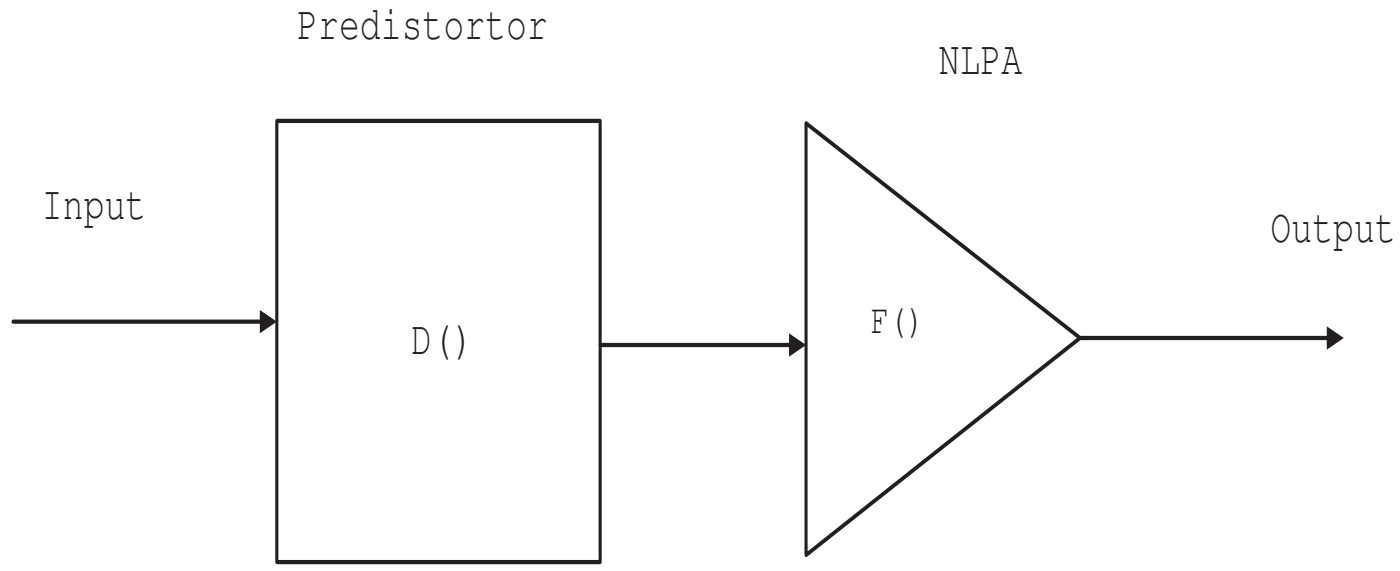

Figure 3.15: Predistortion system model

The predistortion function $F()$, operates on the input signal in a way that its output signal is distorted in a complementary manner to the distortion caused by the nonlinear PA. Therefore, the output signal of the nonlinear PA is the undistorted amplified replica of the input. The procedure can be expressed as:

$$
D(F(x(t)))=A x(t)
$$


Predistortion are aimed at solving both AM-AM and AM-PM correction [21]. In [28] and [29], authors propose different predistortion schemes.

The main drawback of the predistortion is that the technique may need precise knowledge of the PA. Any inaccuracy in the PA model may cause the worse performance of the linearization.

\section{Other Linearization Techniques}

The techniques mentioned above do not present all the possible linearization techniques. Besides the linearization of the nonlinear PA, some other distortion cancellation techniques are also used. In [30], a distortion cancellation scheme at the receiver that can reduce the implementation complexity of the transmitter is introduced. Another method, so called Linear Amplification with Nonlinear Components (LINC) [27] may have an interest. The input signal of LINC amplifier is split into two constant envelope components, each of them is amplified individually. Then the amplified components are fed to an ideal summing junction of recombination. The resulting signal of recombination is an amplified signal of the input with no added distortion.

The main advantages of LINC technique are that it has potential for high efficiency and furthermore, is straightforward to understand [27]. However, it is sensitive to the balance between two amplifiers.

\subsection{Other Impairments}

In addition to RF impairments that have been mentioned above, there are some other impairments that degrade the performance of the OFDM system. In this section, two effects are briefly overviewed.

\subsubsection{Jitter Effects}

In the time domain, jitter is the statistical measurement of variations in the period of the signal, which describes how the signal period wandered from the 
ideal case. There are mainly two kinds of jitter, one depends on the absolute sampling time and the other is time-invariant. Because of the properties of jitter, it can generate sampling time errors, which results in the dominating effect of jitter that limits the achievable SNR [31].

The reason why jitter is not considered in detail in this thesis is that jitter effects can be reduced to those of phase noise. In this thesis the phase noise is taken into account so that jitter effect is not considered separately.

\subsubsection{Carrier Frequency Offsets}

Another impairment of OFDM system is Carrier Frequency Offsets (CFO). During the up and down conversion at the OFDM transceiver, the LO should produce a ideal sine wave at the standard RF carrier frequency. In practice, however, the produced carrier frequency may differ from one to another, which results in the possible CFO between the transmitter and receiver [18].

From [32], it can be noticed that OFDM is sensitive to CFO which destroy orthogonality and cause ICI. However, although CFO and phase noise are caused by different properties of the LO, the effects of both two effects can be seen as similar. Therefore, this effect is not considered in the simulation of the thesis.

\subsection{Summary}

In this Chapter, three crucial dirty RF components are introduced in details and two impairments are introduced briefly. The characteristics and principles of the impairments are overviewed separately. It can be noticed that the performance of the OFDM system suffers from these impairments. The compensation schemes for each impairment are presented as well. We can see that in order to increase the performance of the OFDM system, the dirty RF effects so far have been extensively analyzed mainly separately and techniques for their mitigation have been elaborated separately for decades. In the next Chapter, joint effects of these RF impairments are presented. 


\section{Chapter 4}

\section{Joint Effects of Dirty RF}

This Chapter analyze joint effects of the dirty RF on the OFDM signal. Theoretical equations as well as simulation results are shown to help to understand the joints effects.

\subsection{Joint Effects}

\subsubsection{Introduction}

The joint effects of I/Q Imbalance and phase noise have been studied extensively. In [33]-[35], the system model of OFDM transmission with I/Q imbalance and phase noise is introduced and different compensation schemes are proposed. In [36], a system performance analysis of the joint effects of nonlinear PA and phase noise is presented. In this thesis, based on individual concepts of RF impairments, a theoretical expression of the joint effects model is derived.

An implementation of an OFDM transceiver is depicted in Figure 4.1. The $\mathrm{RF}$ front-end consists of components that need to be carefully considered to ensure a good system performance. At the transmitter, the local oscillator (LO) can cause I/Q imbalance and phase noise. The nonlinear effect of the PA can also generates signal distortion. At the receiver, the receiver LO has the same effect as transmitter LO. Both I/Q imbalance and phase noise are caused by the RF signal down-conversion process. In this thesis, the effects of $\mathrm{I} / \mathrm{Q}$ imbalance and nonlinear distortion of PA at the transmitter along with 
I/Q imbalance and phase noise at the receiver side are jointly analyzed.

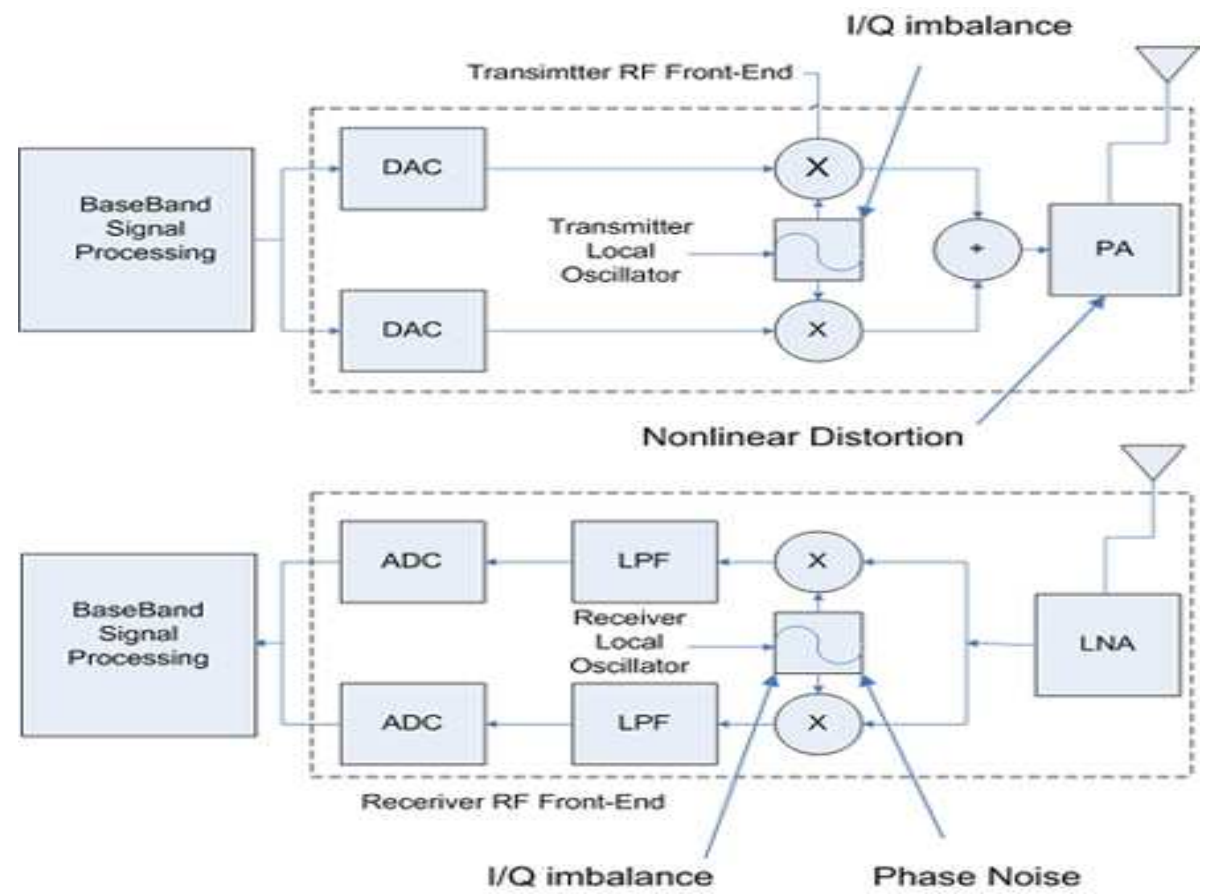

Figure 4.1: Simplified model for OFDM transceiver

\subsubsection{System Model of Joint Effects}

The OFDM signal with the I/Q imbalance can be written as a function of $\left(\mu_{t}, \nu_{t}\right)$

$$
x_{i q}(t)=\mu_{t} x(t)+\nu_{t} x^{*}(t)
$$

where $\mu_{t}$ and $\nu_{t}$ are defined in Eq.(3.7) and (3.8) respectively.

The imbalanced signal $x_{i q}(t)$ is amplified by a nonlinear PA. We use a memoryless model of PA. The amplified signal can be expressed as:

$$
u(t)=F\left(x_{i q}(t)\right)
$$

If $\rho_{i q}(t)$ and $\psi_{i q}(t)$ are the amplitude and phase of $x_{i q}(t)$ respectively, the output of the nonlinear power amplifier can be written as: 


$$
u(t)=F_{A}\left(\rho_{i q}(t)\right) e^{j F_{P}\left(\rho_{i q}(t)\right)} e^{j \psi_{i q}(t)}
$$

where $F_{A}()$ and $F_{P}()$ are the AM-AM and AM-PM conversions of the amplifier.

The output of the nonlinear PA also can be expressed as [22] [36]:

$$
x_{d-i q}(t)=K x_{i q}(t)+d(t)
$$

$K$ can be viewed as a constant number for different certain value of OBO [22]. In such case, $d(t)$ is additive Gaussian-like noise with zero mean, variance $\sigma_{D}^{2}$ can be evaluated by Eq. (3.31).

In the case of phase noise at the receiver, the received signal can be expressed as:

$$
r_{p n}(t)=r(t) e^{j \phi(t)}
$$

where $r(t)$ is the received signal, $\phi(t)$ represents the phase noise process at the receiver, and it is modelled as Wiener process here.

The impact of $\mathrm{I} / \mathrm{Q}$ imbalance on the received signal with phase noise can be modelled as:

$$
r_{i q-p n}(t)=\mu_{r} r_{p n}(t)+\nu_{t} r_{p n}^{*}(t)
$$

Therefore, by using Eq.(4.5) and (4.6) the signal with I/Q imbalance and phase noise can be written as:

$$
r_{i q-p n}(t)=\mu_{r} r(t) e^{j \phi(t)}+\nu_{t} r^{*}(t) e^{-j \phi(t)}
$$

In the following subsections, channel effects are introduced for analysis. 


\section{Additive White Gaussian Noise Channel}

Considering the transmission over AWGN channel, the received signal $r(t)$ can be expressed as:

$$
r(t)=x_{d-i q}(t)+w(t)
$$

where $w(t)$ is the additive Gaussian noise.

By substituting Eq. (4.1) and (4.4) into (4.8), we obtain

$$
r(t)=K \mu_{t} x(t)+K \nu_{t} x^{*}(t)+d(t)+w(t)
$$

If we denote $v(t)=d(t)+w(t)$. Eq.(4.9) can be expressed as:

$$
r(t)=K \mu_{t} x(t)+K \nu_{t} x^{*}(t)+v(t)
$$

Substituting Eq.(4.10) into Eq.(4.7), the received signal before ADC with all $\mathrm{RF}$ impairments can be obtained as

$$
\begin{aligned}
r_{p a-i q-p n}(t)= & \mu_{r}\left(K \mu_{t} x(t)+K \nu_{t} x^{*}(t)+v(t)\right) e^{j \phi(t)} \\
& +\nu_{r}\left(K \mu_{t} x(t)+K \nu_{t} x^{*}(t)+v(t)\right)^{*} e^{-j \phi(t)}
\end{aligned}
$$

Since further baseband signal processing is done digitally, it is reasonable to use sampled down-converted baseband signal $r_{p a-i q-p n}(n)$ to replace the continuous time signal $r_{p a-i q-p n}(t)$. Therefore, the baseband signal can be expressed as:

$$
\begin{aligned}
r_{p a-i q-p n}(n)= & \mu_{r}\left(K \mu_{t} x(n)+K \nu_{t} x^{*}(n)+v(n)\right) e^{j \phi(n)} \\
& +\nu_{r}\left(K \mu_{t} x(n)+K \nu_{t} x^{*}(n)+v(n)\right)^{*} e^{-j \phi(n)}
\end{aligned}
$$

For the sake of simplicity, let us pose $r_{p a-i q-p n}=r_{p a-i q-p n}(n), x=x(n)$, $v=v(n)$, and $\phi=\phi(n)$. After simply manipulations, we obtain

$$
\begin{aligned}
r_{p a-i q-p n}= & \mu_{r}\left(K \mu_{t} x+K \nu_{t} x^{*}+v\right) e^{j \phi}+\nu_{r}\left(K \mu_{t} x+K \nu_{t} x^{*}+v\right)^{*} e^{-j \phi} \\
= & \left(K \mu_{t} \mu_{r} e^{j \phi}+K^{*} \nu_{t}^{*} \nu_{r} e^{-j \phi}\right) x+\left(K \mu_{r} \nu_{t} e^{j \phi}\right. \\
& \left.+K^{*} \mu_{t}^{*} \nu_{r} e^{-j \phi}\right) x^{*}+\left(\mu_{r} v e^{j \phi}+\nu_{r} v^{*} e^{-j \phi}\right) \\
= & \left(a_{1} e^{j \Phi}+a_{2} e^{-j \phi}\right) x+\left(b_{1} e^{j \phi}+b_{2} e^{-j \phi}\right) x^{*}+\left(\mu_{r} v e^{j \phi}+\nu_{r} v^{*} e^{-j \phi}\right)
\end{aligned}
$$


Where $a_{1}=K \mu_{t} \mu_{r}, a_{2}=K^{*} \nu_{t}^{*} \nu_{r}, b_{1}=K \mu_{r} \nu_{t}, b_{2}=K^{*} \mu_{t}^{*} \nu_{r}$.

After OFDM demodulation, the resulting frequency domain signal is given by:

$$
R=\left(a_{1}-a_{2}\right) X I(0)+\left(a_{1}-a_{2}\right) I C I+\left(b_{1}-b_{2}\right) X_{m}^{*} I(0)+\left(b_{1}-b_{2}\right) I C I_{m}^{*}+\xi
$$

This result gives the input-output relation in an OFDM system with both transmitter and receiver dirty RF effects when signal is transmitted over AWGN channel. It is worth noting, however, that $\xi$ and $X$ are correlated.

\section{Fading Channel}

We now consider OFDM transmission over a frequency selective fading channel. $h(t)$ is the channel impulse response. Let be the frequency response of channel. Under the standard assumption, the length of the channel impulse response is shorter than the OFDM CP. The received signal can be expressed as:

$$
r(t)=h(t) \otimes x_{d-i q}(t)+w(t)
$$

After several manipulations similar to the AWGN case, the received signal with all RF impairments can be obtained as:

$$
\begin{aligned}
r_{p a-i q-p n}= & \mu_{r}\left(K \mu_{t} h \otimes x+K \nu_{t} h^{*} \otimes x^{*}+v\right) e^{j \phi}+\nu_{r}\left(K \mu_{t} h \otimes x+K \nu_{t} h^{*} \otimes x^{*}+v\right)^{*} e^{-j \phi} \\
= & \left(K \mu_{t} \mu_{r} e^{j \phi}+K^{*} \nu_{t}^{*} \nu_{r} e^{-j \phi}\right) h \otimes x+\left(K \mu_{r} \nu_{t} e^{j \phi}+K^{*} \mu_{t}^{*} \nu_{r} e^{-j \phi}\right) h^{*} \otimes x^{*} \\
& +\left(\mu_{r} v e^{j \phi}+\nu_{r} v^{*} e^{-j \phi}\right) \\
= & \left(a_{1} e^{j \phi}+a_{2} e^{-j \phi}\right) h \otimes x+\left(b_{1} e^{j \phi}+b_{2} e^{-j \phi}\right) h^{*} \otimes x^{*} \\
& +\left(\mu_{r} v e^{j \phi}+\nu_{r} v^{*} e^{-j \phi}\right)
\end{aligned}
$$

where $a_{1}, a_{2}, b_{1}$ and $b_{2}$ are defined as same as those in Eq.(4.13).

After OFDM demodulation, the resulting frequency domain signal is given by:

$$
R=\left(a_{1}-a_{2}\right) X H I(0)+\left(a_{1}-a_{2}\right) I C I_{h}+\left(b_{1}-b_{2}\right) X_{m}^{*} H_{m}^{*} I(0)+\left(b_{1}-b_{2}\right)\left(I C I_{h}\right)_{m}^{*}+\xi
$$


In Eq.(4.14) and (4.17), three RF impairments have been considered in the OFDM baseband signal. It is clear that PA nonlinearity introduces distortion factor $K$ and $d(n)$, the I/Q imbalance at both transmitter and receiver bring $\mu_{t}, \mu_{r}, \nu_{t}$ and $\nu_{r}$ to the subcarrier signal and the mirror frequency subcarrier signal. I/Q imbalance causes ICI because the power that leaks from the signal on the the mirror frequency subcarriers are under consideration. Meanwhile, the phase noise effect introduces a CPE when $I(0)=e^{j \theta}$, as well as ICI [35].

\subsection{Simulation Results}

\subsubsection{Simulation Description}

Figure 4.2 shows a block diagram of the simulator.The modulation method is M-QAM and number of subcarrier is $\mathrm{N}$ which is exponent of 2. Then $\mathrm{I} / \mathrm{Q}$ imbalance is inserted as the main RF impairment of the transmitter LO. The amplification is done by applying the TWTA and SSPA models described in the last chapter. The OBO value is changed by turning the input power and the saturation level is set as constant.

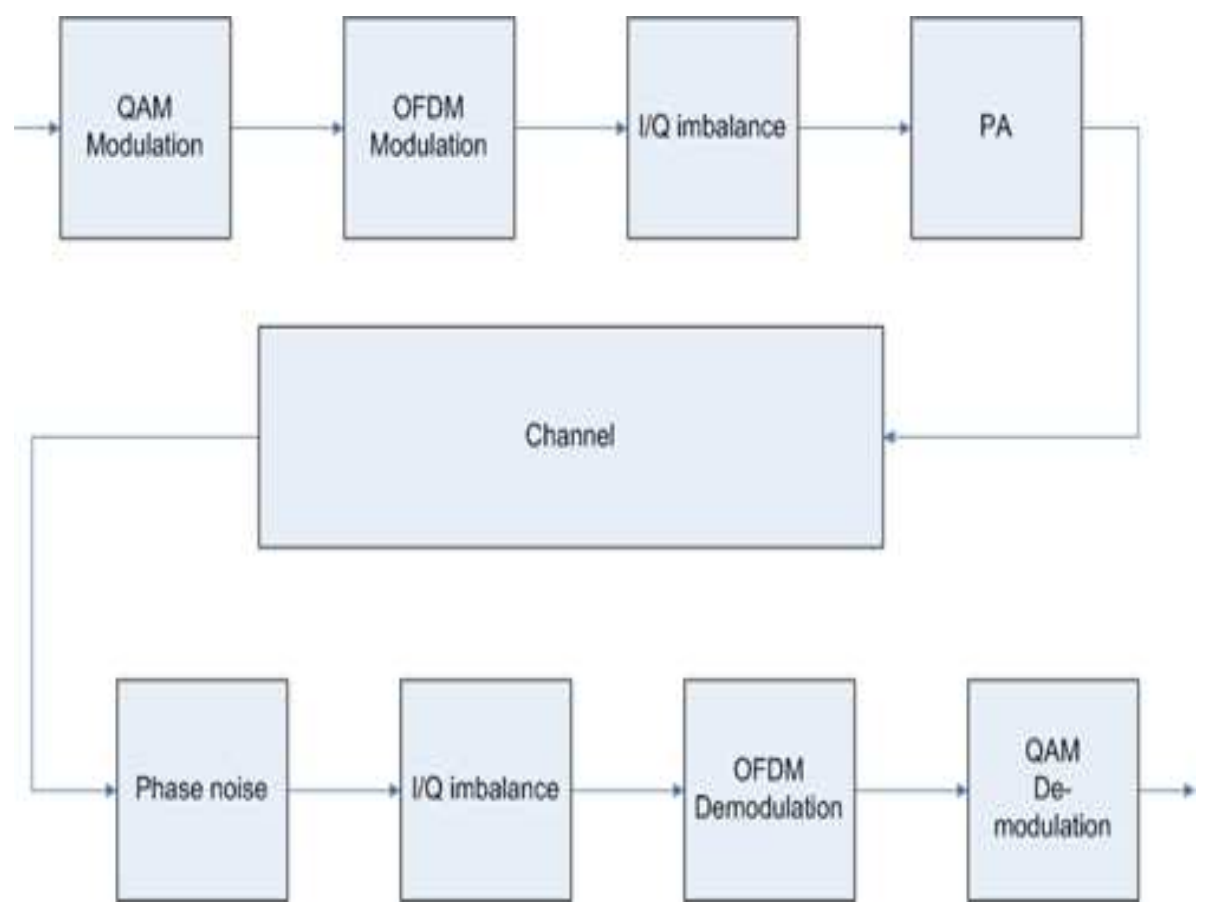

Figure 4.2: Block diagram of simulator 
The channel is modelled as both AWGN channel and 4-tap Rayleigh fading channel. After signal passing through the channel, phase noise which is modelled as Wiener process and I/Q imbalance are added to the received signal as the impairments of the receiver LO. FFT and demodulation are used to do the baseband signal processing.

Our simulation results are presented in the following subsections. First of all, the effects of the RF impairments are shown separately. Then joint effects are given.

\subsubsection{I/Q Imbalance}

First, Figure 4.3 shows the signal constellation map with an I/Q imbalance. 16-QAM is used and the number of subcarriers is 64 . The parameters of $\mathrm{I} / \mathrm{Q}$ imbalance are $\triangle \varphi_{t}=\Delta \varphi_{r}=5^{\circ}, \gamma_{t}=\gamma_{r}=0.05$.

The effects of I/Q imbalance in both the AWGN and Rayleigh fading channel are studied. 4-QAM, 16-QAM and 64-QAM are presented with 64 subcarriers and CP length is 16. $\triangle \varphi_{t}=\triangle \varphi_{r}=5^{\circ}, \gamma_{t}=\gamma_{r}=0.05$. BER results are reported in Figure 4.4 and 4.5. Reference curves in the Figures 4.4 and 4.5 are given for a OFDM system with the perfect $\mathrm{I} / \mathrm{Q}$ match. We can notice that as the number of symbol alphabet of QAM increases, i.e. from 4 to 64, the performance of the system suffers more from I/Q imbalance.

Next comes analysis of the I/Q imbalance with different parameters. With today's technology, 0.01-0.02 amplitude imbalance and $1^{\circ}-2^{\circ}$ phase imbalance is realistic [36]. Therefore, values close to or larger than the realistic value are chosen to see how I/Q imbalance affects the system performance. The BER curves for phase imbalance of $3^{\circ}, 5^{\circ}$, and $10^{\circ}$ are shown in Figure 4.6. The signal is 16-QAM symbol and the BER is obtained for the signal passing through the AWGN channel. We can notice that when phase imbalance is $3^{\circ}$, the degradation is around $1 \mathrm{~dB}$ at $\mathrm{BER}=10^{-4}$. The degradation is around 3 $\mathrm{dB}$ for $5^{\circ}$ phase imbalance. We can see that when phase imbalance is $10^{\circ}$, for 16-QAM, the BER performance is very poor. 


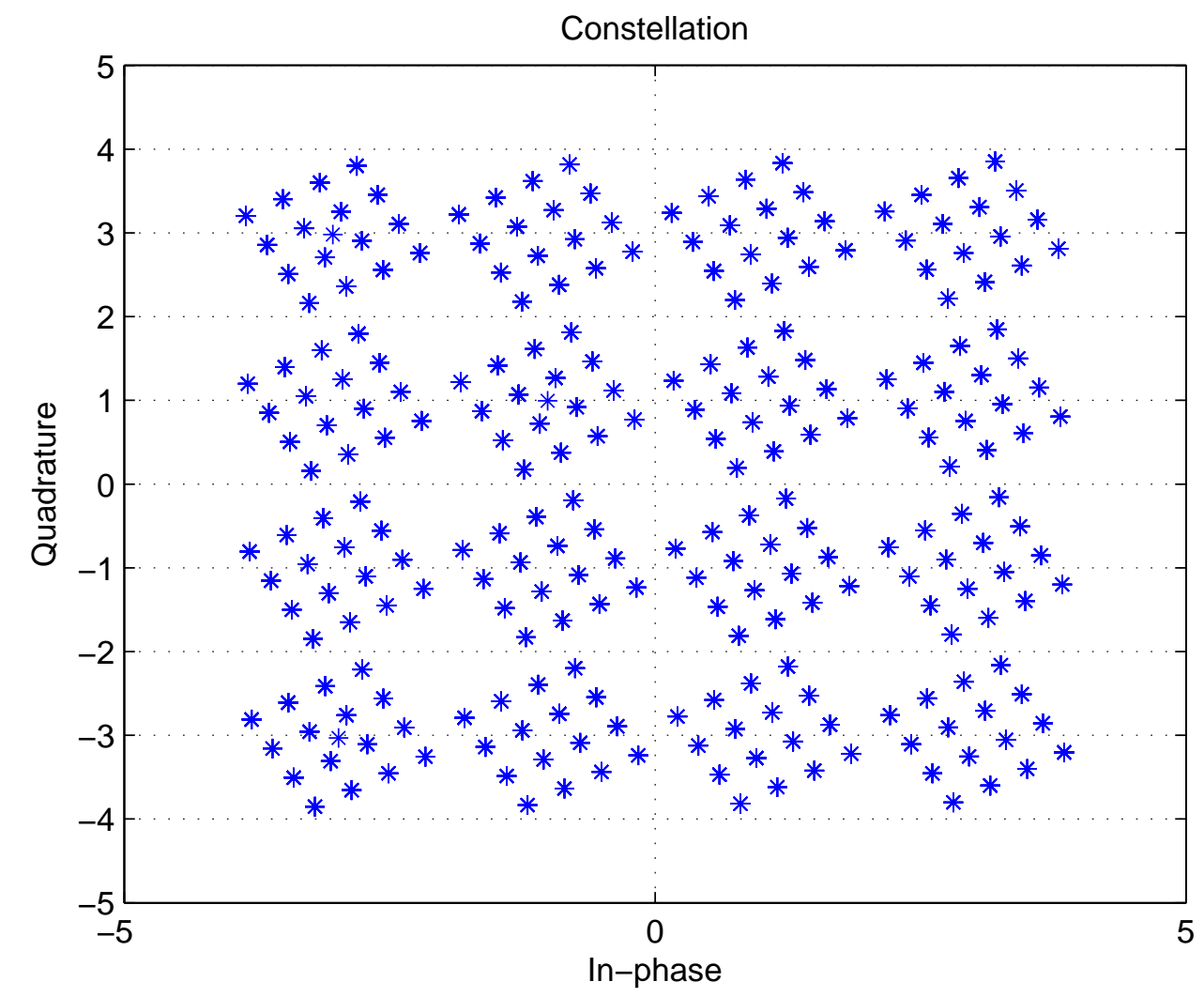

Figure 4.3: Effect of I/Q imbalance on the signal constellation

When there is only amplitude imbalance, the BER can be obtained in the same way, and the result is shown in Figure 4.7. If the amplitude imbalance is less than 0.05 , the degradation is less then $1 \mathrm{~dB}$ at $\mathrm{BER}=10^{-2}$; even at $\mathrm{BER}=10^{-4}$, the degradation is only slightly more than $1 \mathrm{~dB}$. However, for the case that amplitude imbalance is 0.10 , the degradation is greater than 4 $\mathrm{dB}$ even for $\mathrm{BER}=10^{-2}$. The performance is unacceptable for 0.15 amplitude imbalance.

Figures 4.8 and 4.9 show the PDF histograms of the interference caused by the I/Q imbalance. Figure 4.8 presents the PDF in time domain. It can be seen that in the time domain, the PDF of the real and imaginary part of interference follows Gaussian distribution and the PDF of the absolute value follows Rayleigh distribution. Thus, the interference in the time domain can be viewed as Gaussian. However, in the frequency domain, the interference is not Gaussian even in the case of passing through AWGN channel as we can see from Figures 4.9 and 4.10. Since most of analysis and future work are done 


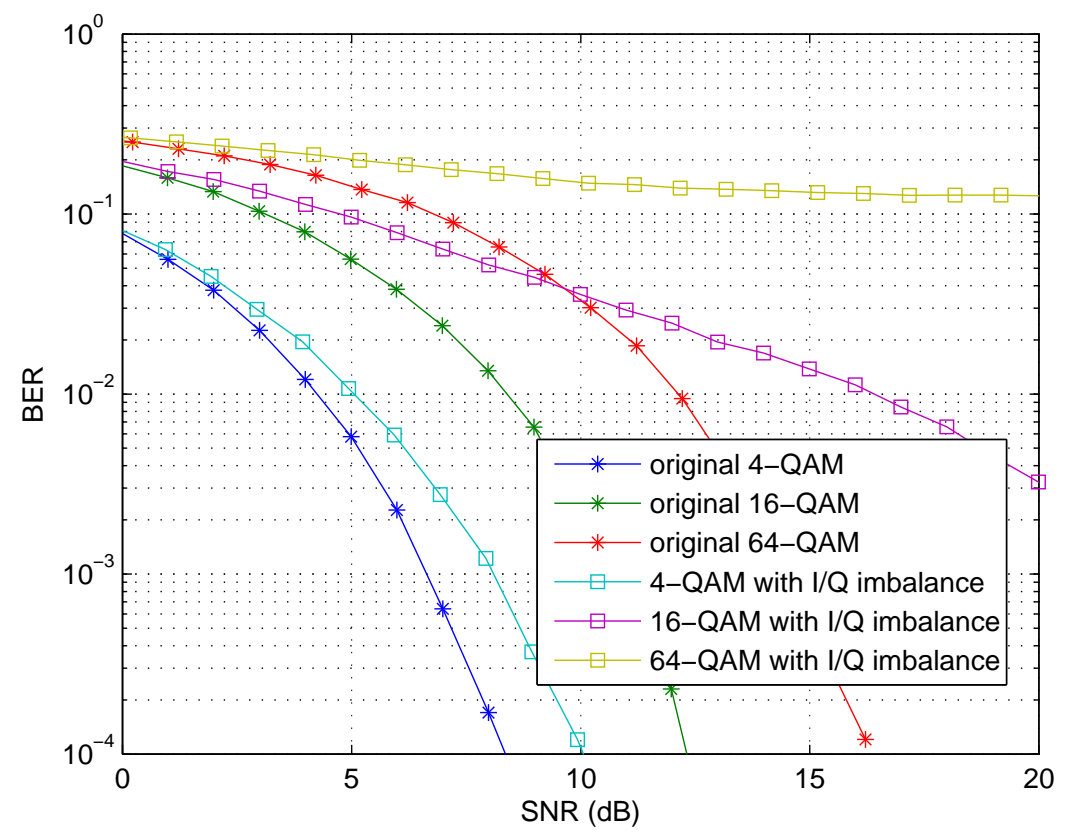

Figure 4.4: BER performance of OFDM system with I/Q imbalance at transmitter and receiver, AWGN channel

in the frequency domain, the analysis of PDF in the time domain cannot be helpful further study. 


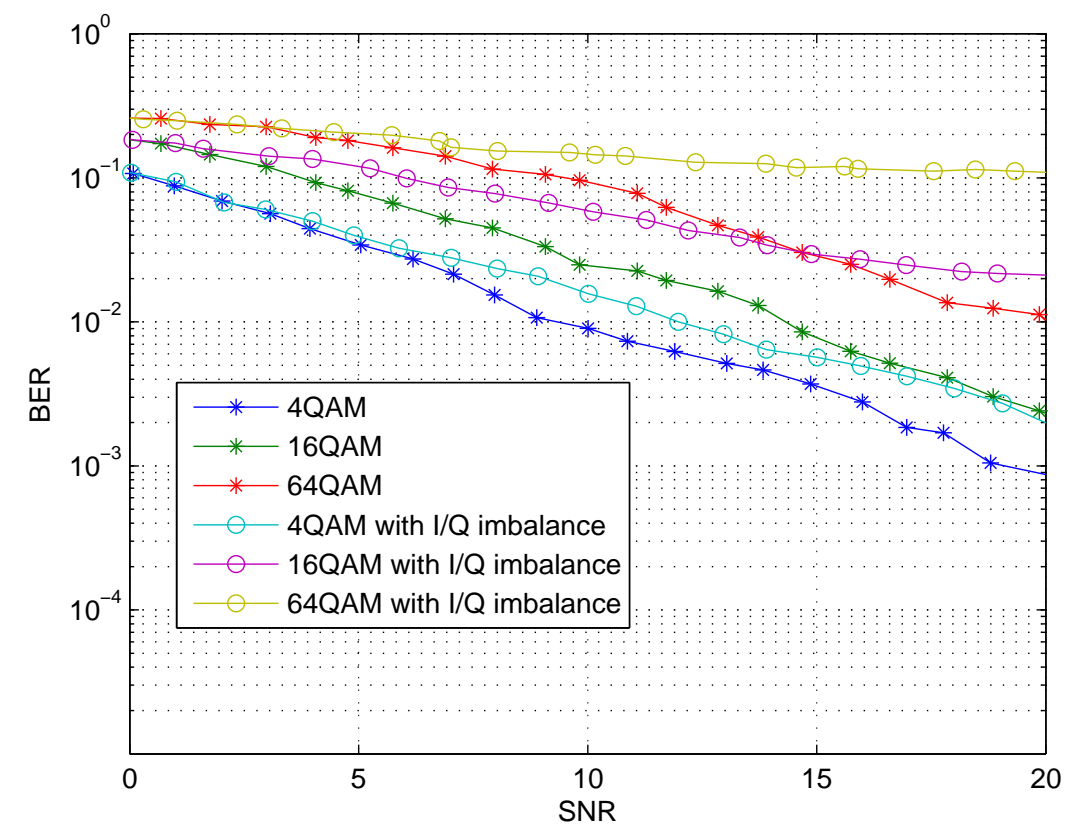

Figure 4.5: BER performance of OFDM system with I/Q Imbalance at transmitter and receiver, 4-tap Rayleigh fading channel

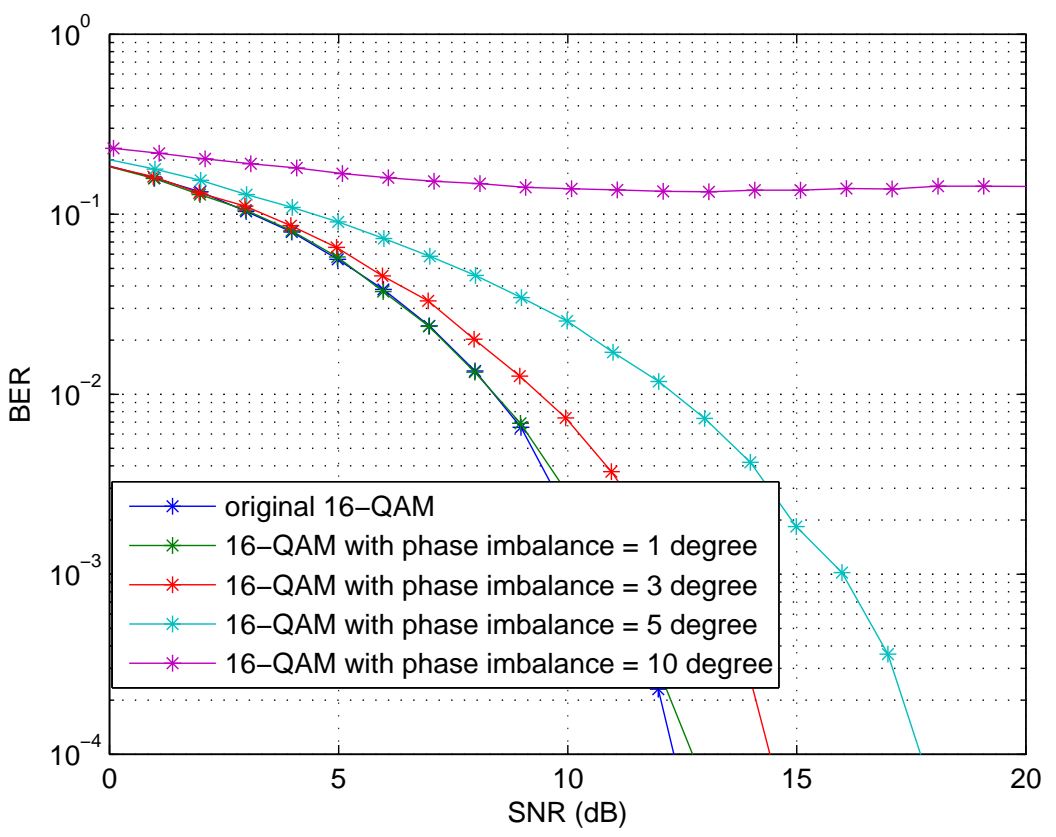

Figure 4.6: BER performance of OFDM system with different phase imbalance parameters, AWGN channel 


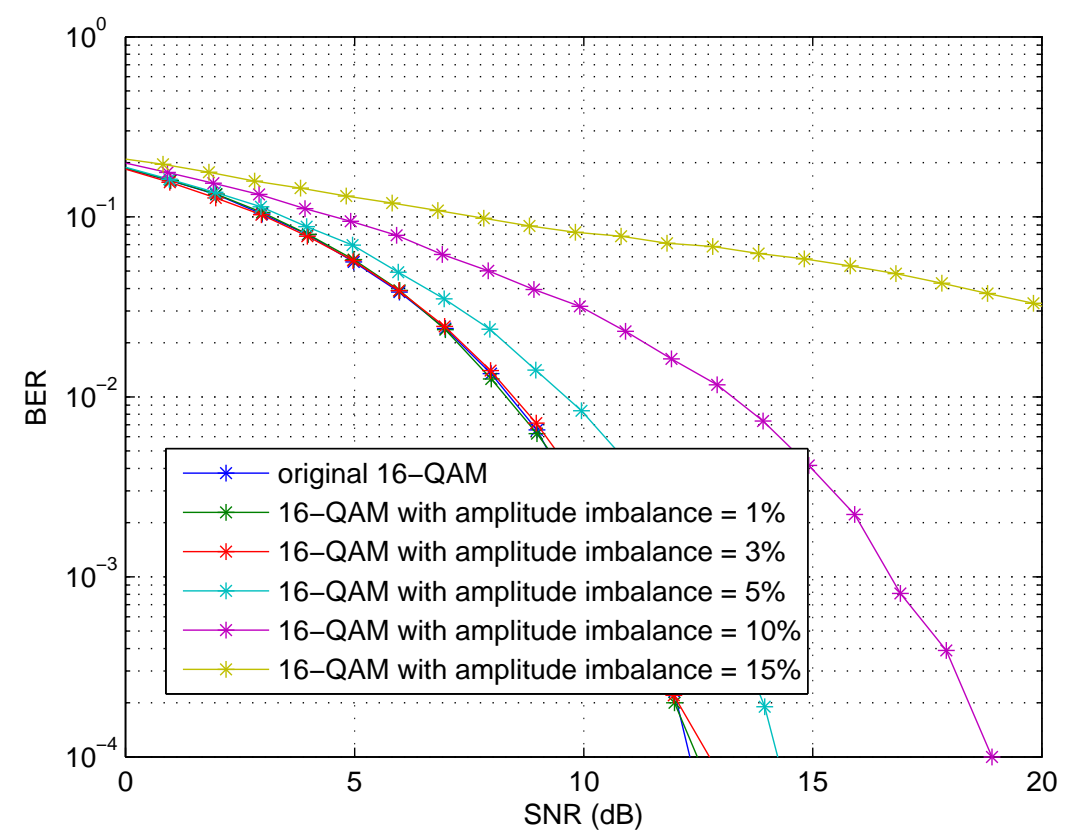

Figure 4.7: BER performance of OFDM system with different amplitude imbalance parameters, AWGN channel
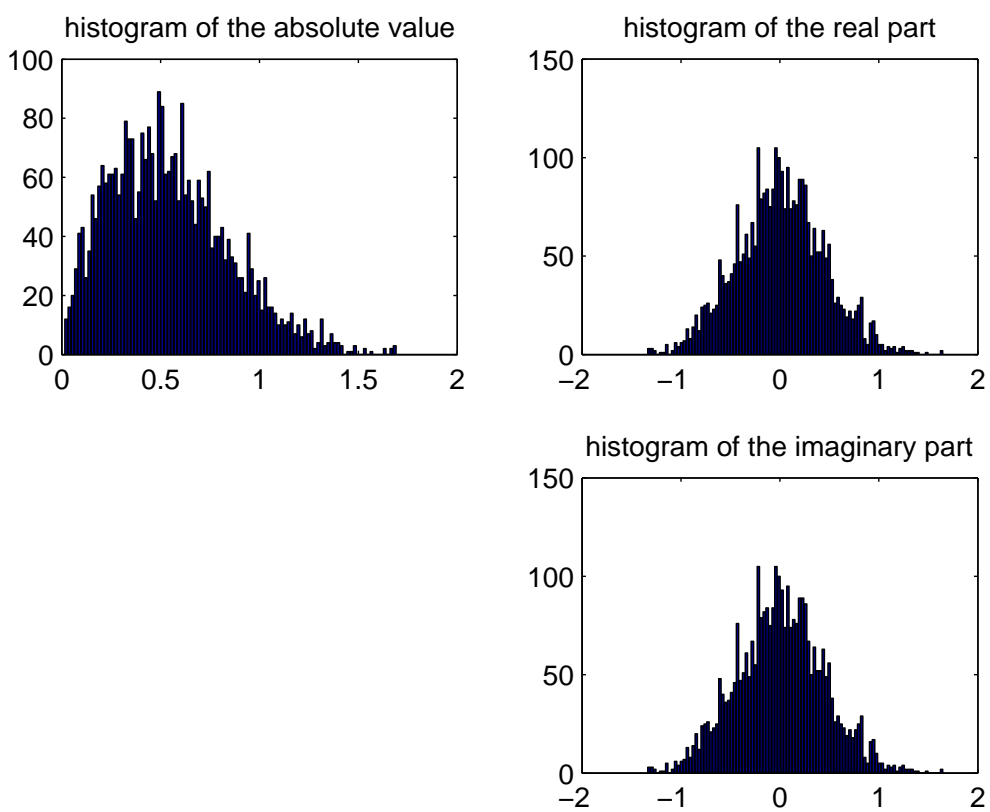

Figure 4.8: Time domain histograms of interferences caused by I/Q imbalance 

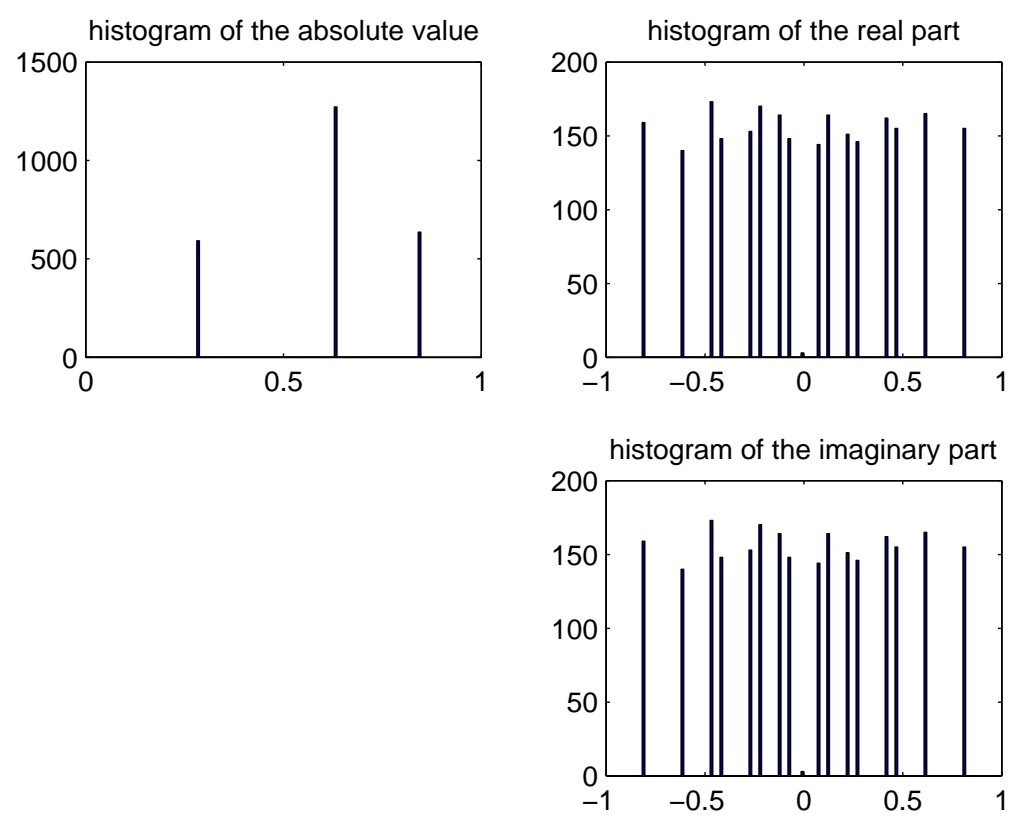

Figure 4.9: Frequency domain histograms of interferences caused by I/Q imbalance
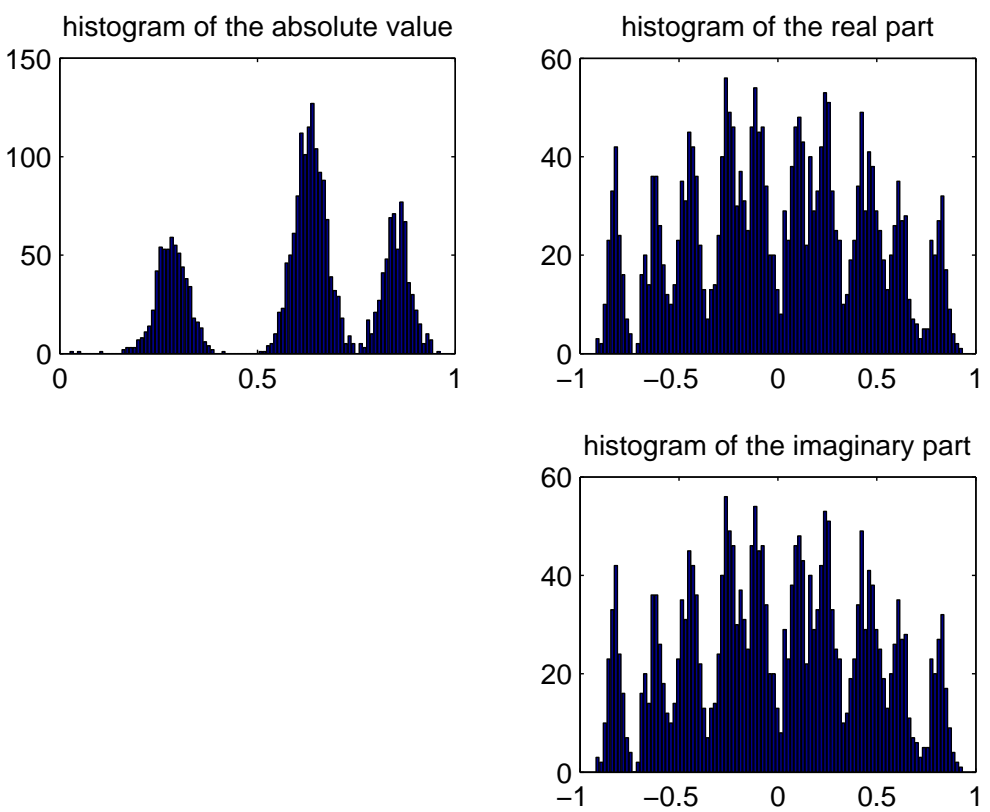

Figure 4.10: Frequency domain histograms of interferences caused by $\mathrm{I} / \mathrm{Q}$ imbalance, AWGN channel, $\mathrm{SNR}=35 \mathrm{~dB}$ 


\subsubsection{Phase Noise}

In this part, the performance degradation caused by phase noise is shown. The impact on the constellation map can be found in the Figure 3.1. Figures 4.11 and 4.12 show the BER performance of the OFDM system with phase noise whose variance is 0.04 in AWGN and Rayleigh fading channel respectively. Since phase noise is modelled as Wiener process, the variance of phase noise is corresponding to the variance of Wiener process. 0.04 is one normal value that is used in some literatures, i.e. in [12]. 4-QAM, 16-QAM and 64-QAM are presented with 64 subcarriers.

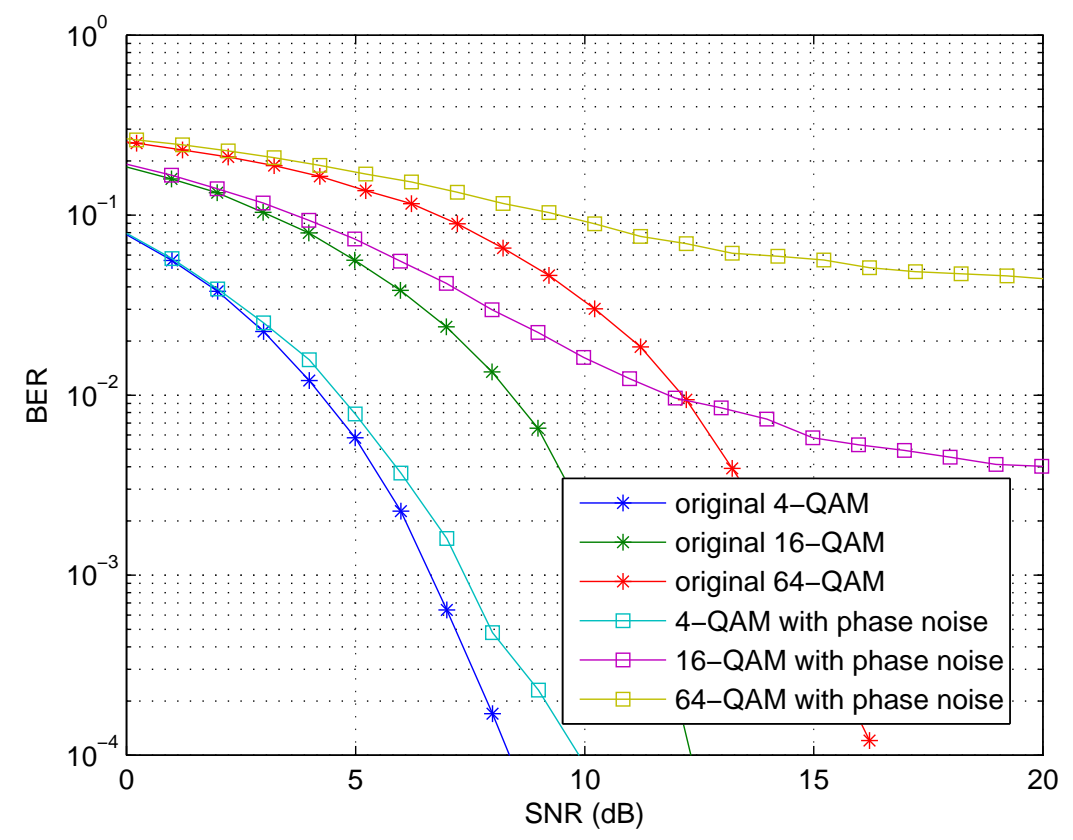

Figure 4.11: BER performance of OFDM system with phase noise, variance $=$ 0.04. AWGN channel

For 16-QAM modulation scheme, the BER is shown in Figure 4.13. Different curves correspond to different values of variance of the phase noise which depends on the oscillator linewidth and symbol period [37].It has been assumed that the OFDM transmission is only impaired by phase noise and additive Gaussian noise. It is apparent that the performance strongly degrades for variance $>0.04$. 


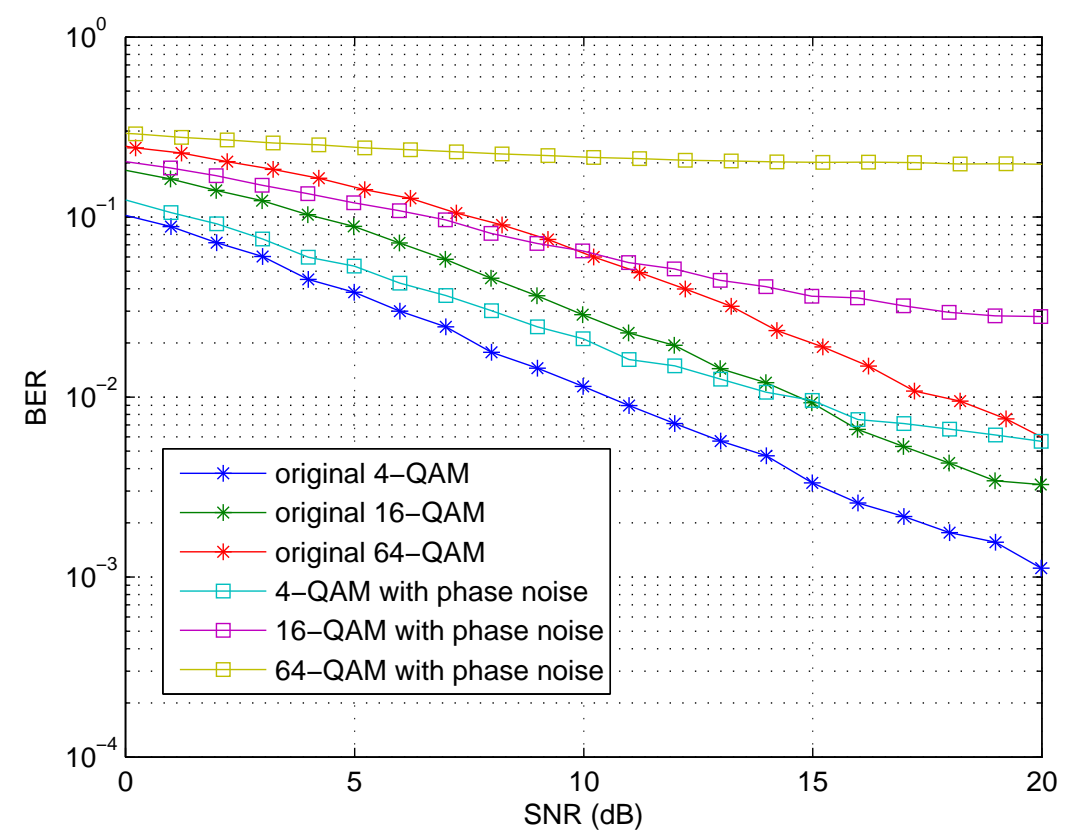

Figure 4.12: BER performance of OFDM system with phase noise, variance = 0.04. 4-tap Rayleigh fading channel

Figures 4.14 - 4.17 show the PDF histograms of interference caused by phase noise in the frequency domain. The variance of phase noise in each figure is different, from 0.02 to 0.6 . It can be observed that when the variance is small, the PDF of the interference can be viewed as Gaussian, which is stated and proved in some literature as well, i.e. in [37]. In Figure 4.17, it can be see that when the variance is 0.6 , the PDF of the interference does not follow Gaussian distribution. 


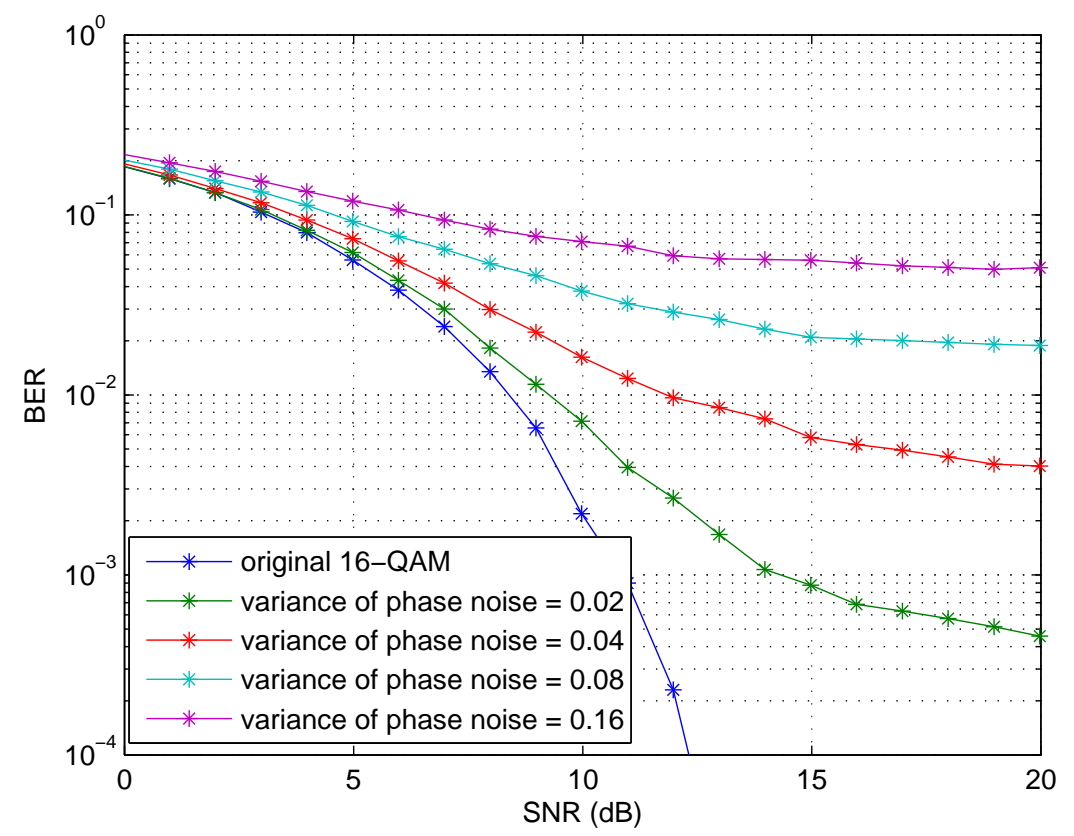

Figure 4.13: BER performance of OFDM system with phase noise, different variance, 16-QAM, AWGN channel
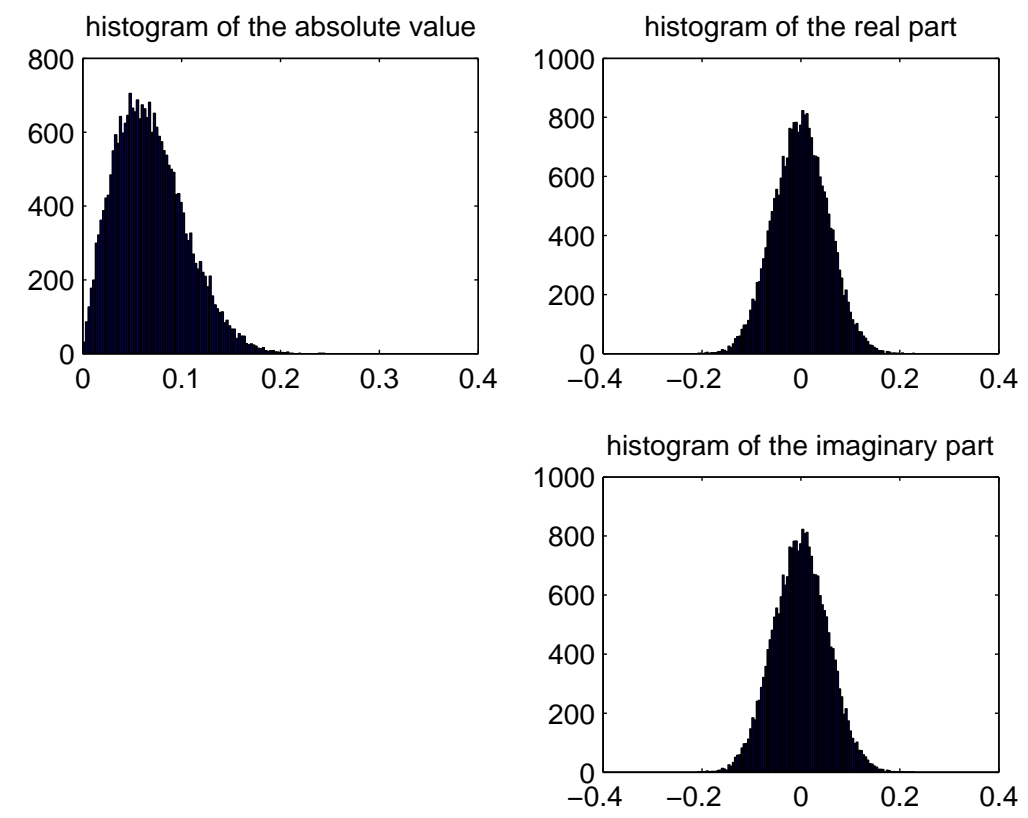

Figure 4.14: Frequency domain histograms of interferences caused by phase noise, variance $=0.02$ 

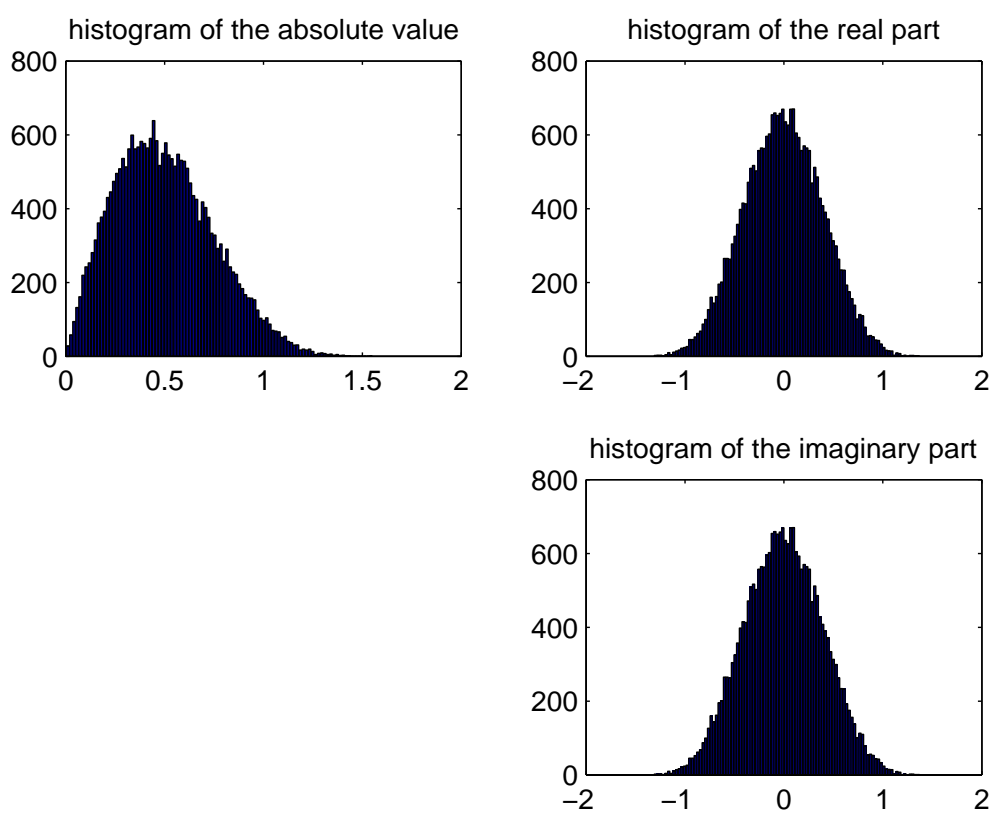

Figure 4.15: Frequency domain histograms of interferences caused by phase noise, variance $=0.2$
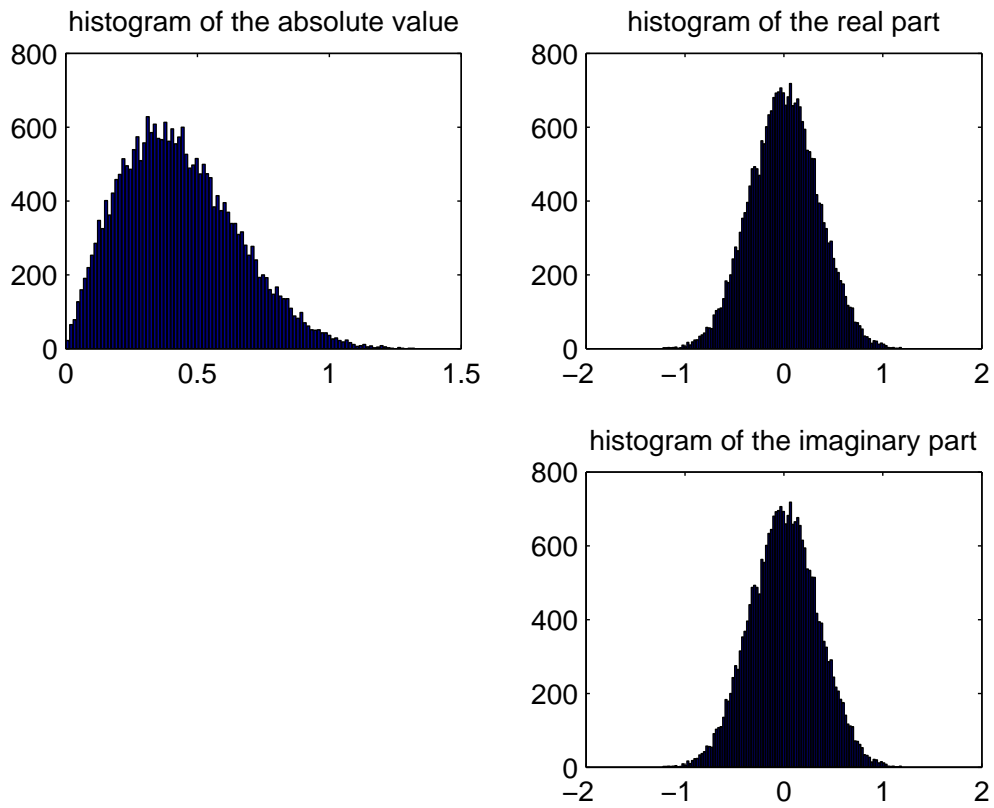

Figure 4.16: Frequency domain histograms of interferences caused by phase noise, variance $=0.4$ 

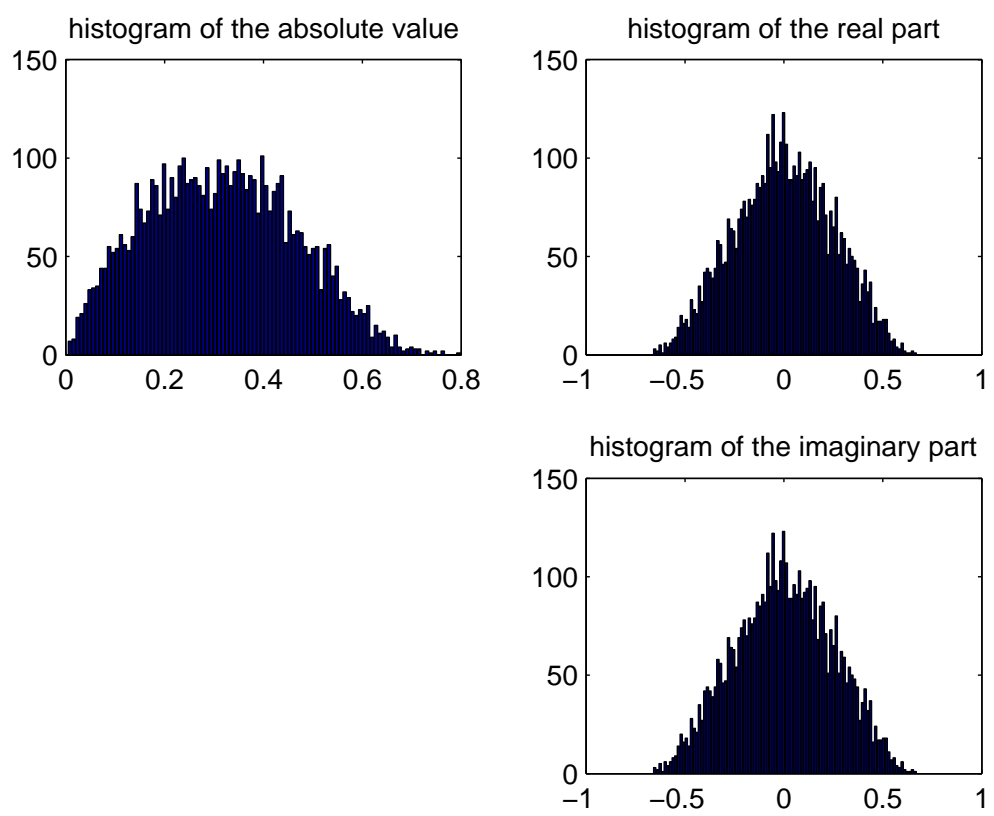

Figure 4.17: Frequency domain histograms of interferences caused by phase noise, variance $=0.6$ 


\subsubsection{Nonlinear Power Amplifier}

Two PA models are used in the simulation here, SSPA and TWTA. The parameters of SSPA are $p=1, A_{o}=1.5$. The parameters of TWTA are $\alpha=\pi / 3, \beta=1$ and $A_{\text {sat }}=3$. Figure 4.18 shows the BER for OFDM system with SSPA, different OBO values. 4-QAM signaling is used here with 64 subcarriers.

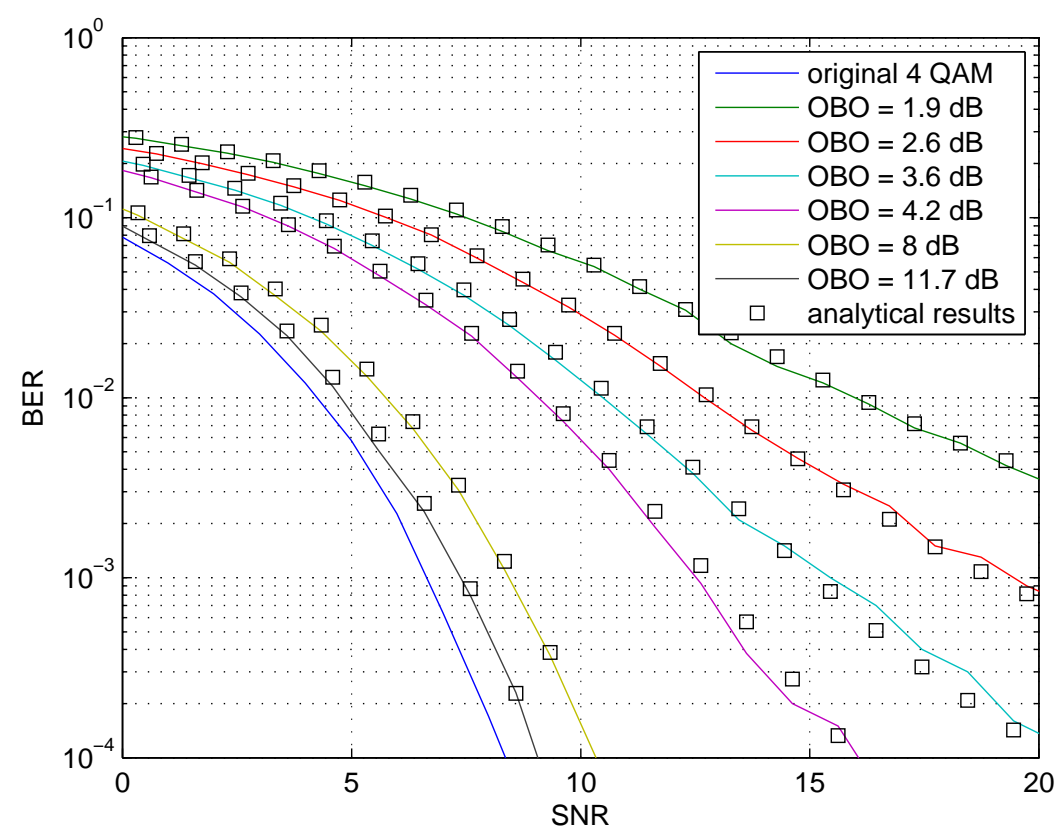

Figure 4.18: BER for OFDM system with SSPA, different OBO values, AWGN channel

In Figure 4.18, some analytical results are shown. These analytical results are obtained based on an equation that is presented in [22]. In this case, analytical and simulation results are in good agreement.

Figure 4.19 shows the BER for OFDM system with TWTA. the modulation scheme is also 4-QAM with 64 subcarriers. In Figure 4.19, some analytical results are shown for different OBO values as well.

It can be seen from Figures 4.18 and 4.19 , when $\mathrm{OBO}=11.7 \mathrm{~dB}$ for OFDM with SSPA and OBO = $11.4 \mathrm{~dB}$ for OFDM with TWTA, the BER performance of the systems are almost as same as the systems without PA nonlinearity. Furthermore, it can also be noticed that when $\mathrm{OBO}<3.4 \mathrm{~dB}$, the performance 


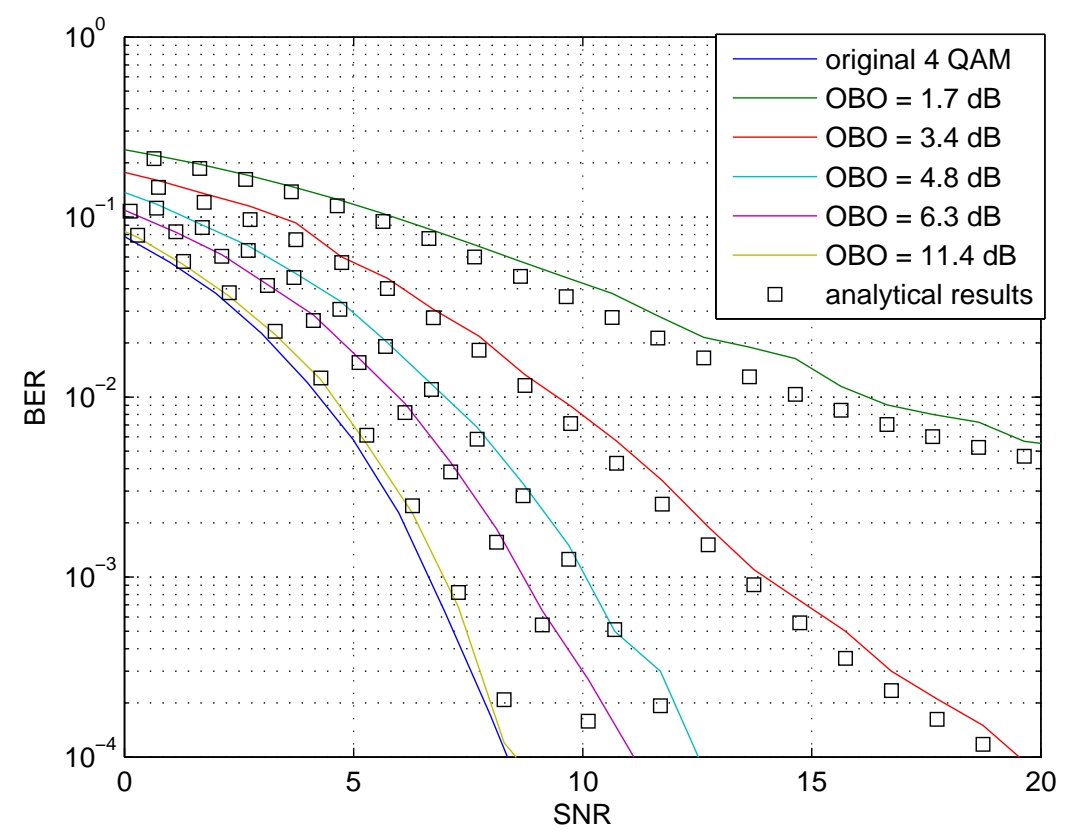

Figure 4.19: BER for OFDM system with TWTA, different OBO values,AWGN channel

degrades seriously for OFDM with SSPA. The same thing happens when OBO $<4.8 \mathrm{~dB}$ for the case that TWTA is used. The figures also show that for the same small value of OBO, the system with the SSPA performs better than the one with TWTA.

Figures 4.20 - 4.22 show the Frequency Domain PDF histograms of Interferences caused by SSPA with different OBO values. According to [22], the interference is zero mean additive Gaussian. It can be observed that as the OBO value increases, the PA works more like a linear model and the variance of noise, $\sigma_{D}^{2}$ become smaller. 

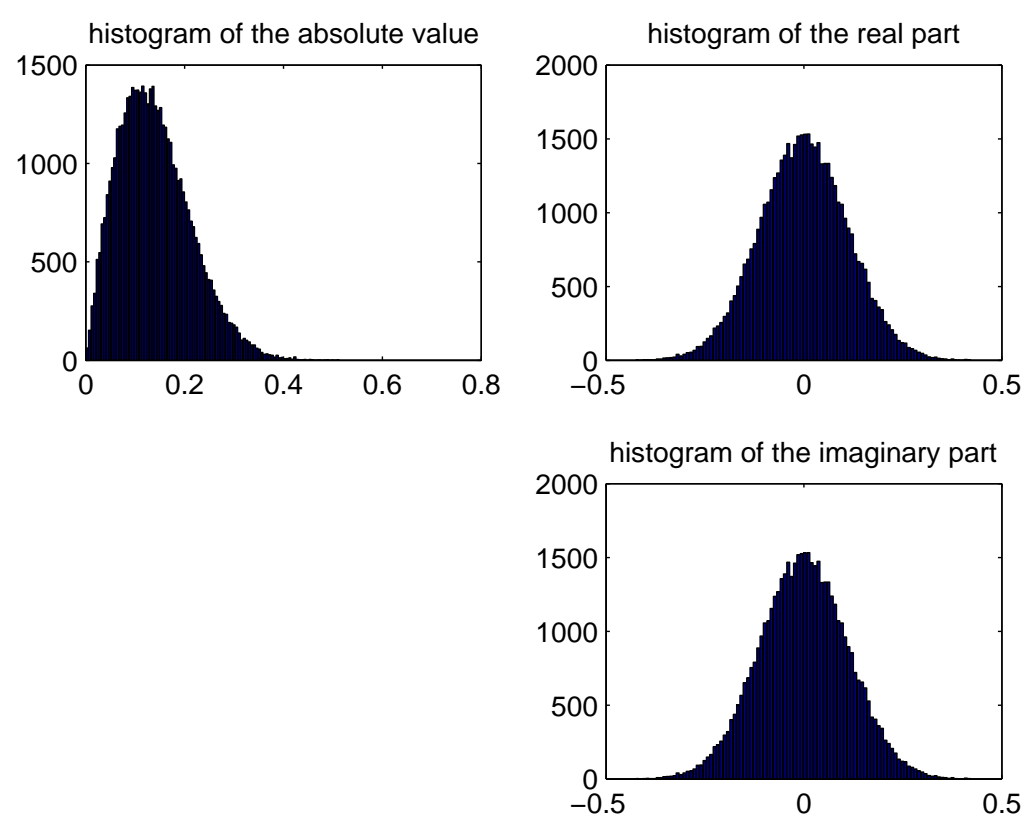

Figure 4.20: Frequency domain histograms of interferences caused by SSPA, $\mathrm{OBO}=1.7 \mathrm{~dB}$
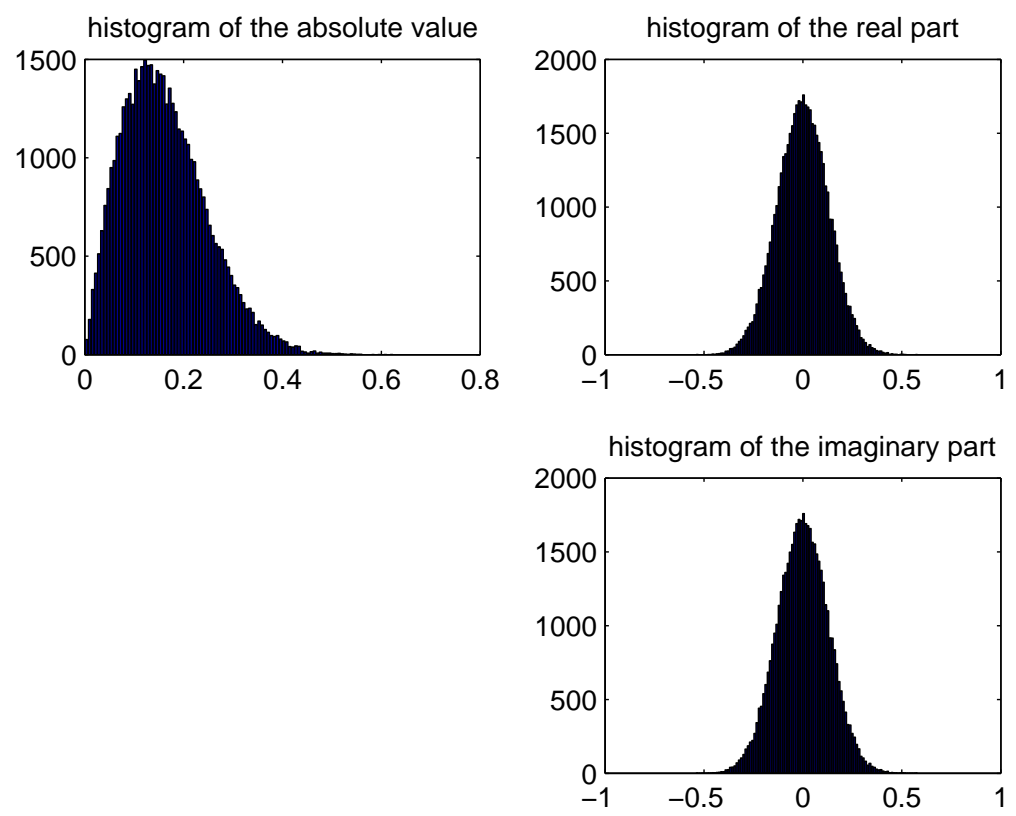

Figure 4.21: Frequency domain histograms of interferences caused by SSPA, $\mathrm{OBO}=4.2 \mathrm{~dB}$ 

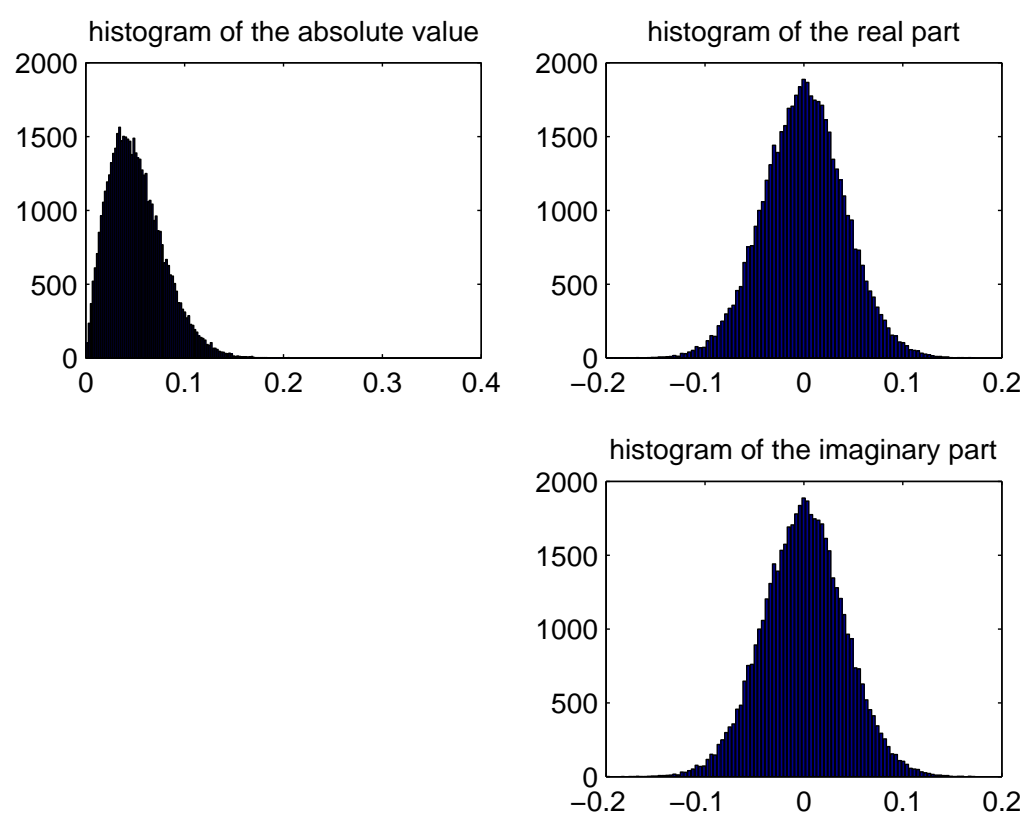

Figure 4.22: Frequency domain histograms of interferences caused by SSPA, $\mathrm{OBO}=11.5 \mathrm{~dB}$ 


\subsubsection{Joint Effects}

In this subsection, the simulation results of the joint effects are shown, and illustrations and discussions are made to analyze the joint effects.

Figure 4.23 shows the BER performance of the OFDM system with dirty RF, including $\mathrm{I} / \mathrm{Q}$ imbalance, phase noise and nonlinear PA. The parameters are $\triangle \varphi_{t}=\Delta \varphi_{r}=5^{\circ}, \gamma_{t}=\gamma_{r}=0.05$ for $\mathrm{I} / \mathrm{Q}$ imbalance, variance of the phase nosie is 0.04. SSPA model is used with $\mathrm{OBO}=4.2 \mathrm{~dB}$ for 4 -QAM, OBO $=$ $3.8 \mathrm{~dB}$ for $16-\mathrm{QAM}$ and $\mathrm{OBO}=4.0 \mathrm{~dB}$ for 64-QAM. As shown in Figure 4.23, dirty RF effects severely degrade the performance even in the 4-QAM case. For 16-QAM and 64-QAM, the BER performances are totally unacceptable. The same performance can be found in Figure 4.24, in which a Rayleigh fading channel is considered. Figure 4.25 shows the signal constellation map of 16QAM. The parameters of I/Q imbalance and phase noise are the same as those in Figure 4.23 , but the $\mathrm{OBO}=7.2 \mathrm{~dB}$ for the nonlinear PA. In such a case, it is possible to notice the rotation and attenuation caused by phase noise, the interference caused by I/Q imbalance, phase noise and PA nonlinearity.

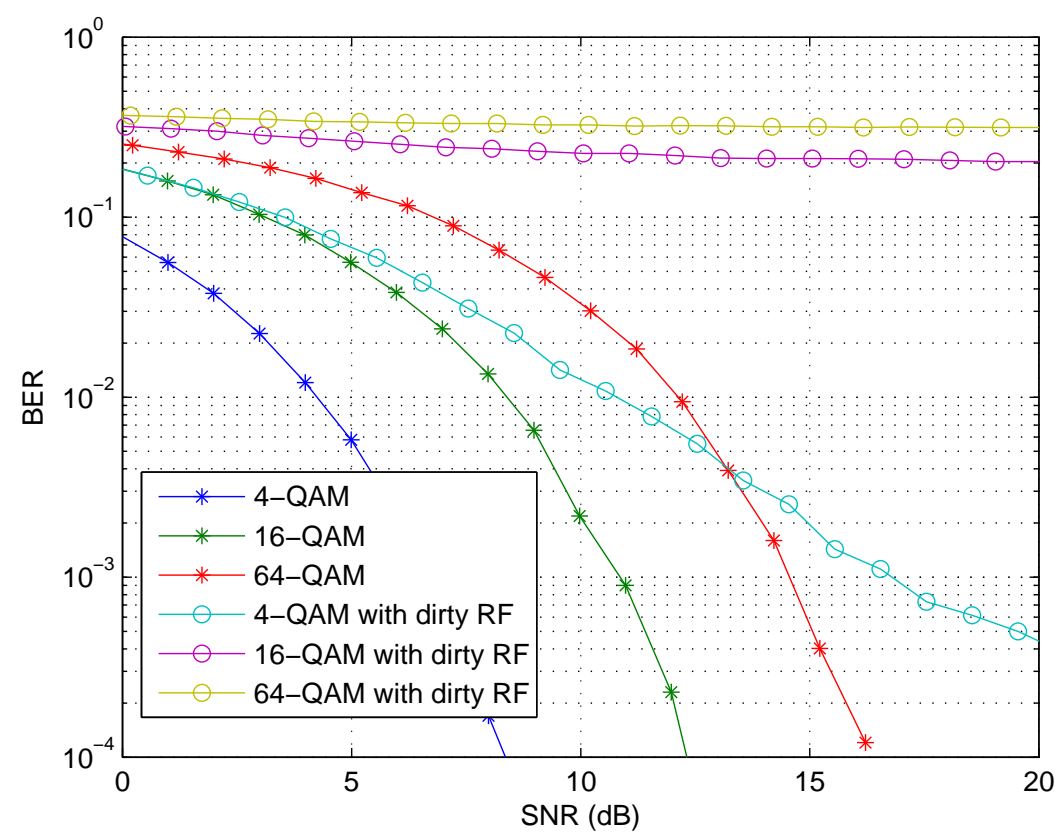

Figure 4.23: BER performance of OFDM system with dirty RF, AWGN channel 


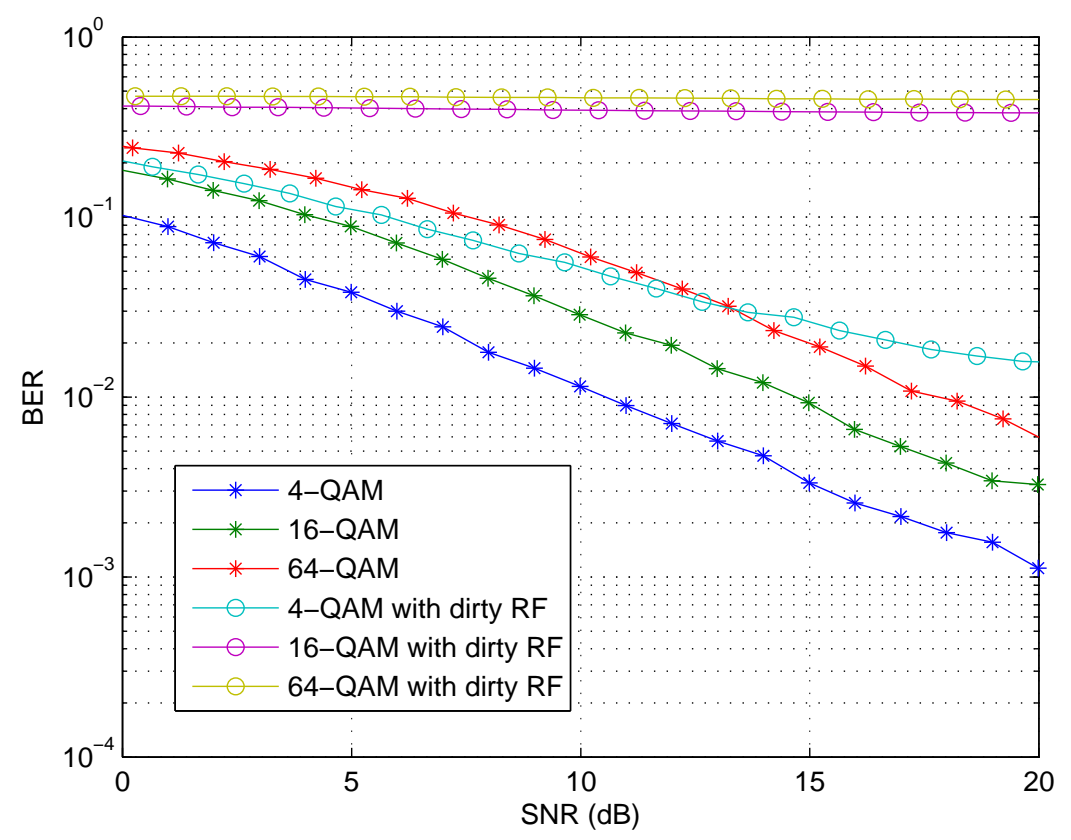

Figure 4.24: BER performance of OFDM system with dirty RF, 4-tap Rayleigh fading channel

Next, we change parameters of one RF impairment to see how individual RF impairment affects the system when considering whole dirty RF effects. Figure 4.26 shows BER performance of the system with different parameters of I/Q imbalance. We consider the same I/Q imbalance for both transmitter and receiver. The modulation scheme is 4 -QAM. The variance of phase noise is 0.04 and SSPA is used with $\mathrm{OBO}=4.3 \mathrm{~dB}$.

Figure 4.26 demonstrates that when the $\triangle \varphi_{t}=\Delta \varphi_{r}<5^{\circ}, \gamma_{t}=\gamma_{r}<0.05$, the degradation is not so strong, i.e. for $\mathrm{BER}=10^{-3}$, the degradation is slightly more than $3 \mathrm{~dB}$. However, when $\triangle \varphi_{t}=\triangle \varphi_{r}=10^{\circ}, \gamma_{t}=\gamma_{r}=0.10$, the degradation is much worse than others, i.e. when BER $=10^{-2}$ in Figure 4.26, the degradation is more than $7 \mathrm{~dB}$ comparing to no I/Q imbalance system. In Figure 4.27 , we can see that the degradation when $\triangle \varphi_{t}=\Delta \varphi_{r}<5^{\circ}, \gamma_{t}=\gamma_{r}<0.05$ is larger that the one in AWGN case. If the 0.01-0.02 amplitude imbalance and $1^{\circ}-2^{\circ}$ phase imbalance is realistic, from Figure 4.26, the degradation caused by $\mathrm{I} / \mathrm{Q}$ imbalance can be thought of as being very slight.

Figures 4.28 - 4.30 show the frequency domain PDF histograms of the interference caused by the joint effects when different I/Q imbalance parameters are 


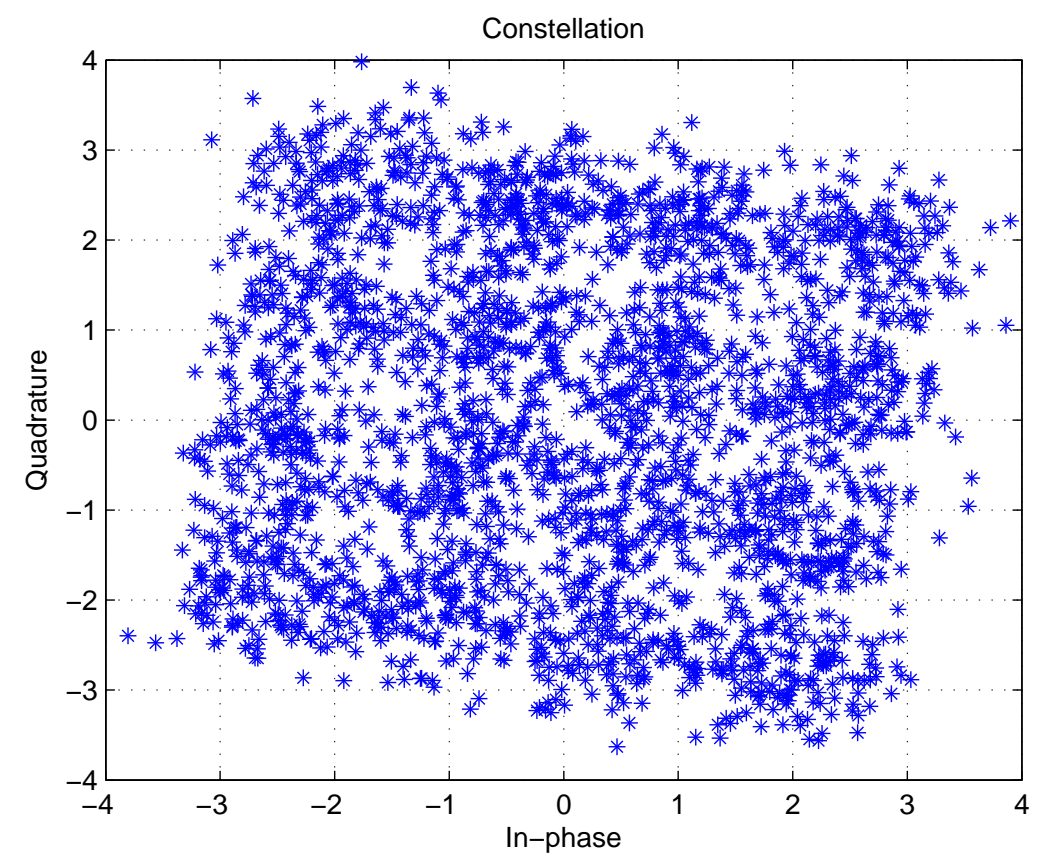

Figure 4.25: Constellation of OFDM signal with dirty RF

used. It can be observed that when amplitude imbalance and phase imbalance are small, the PDF is Gaussian like. As mentioned before, the realistic values are 0.01-0.02 amplitude imbalance and $1^{\circ}-2^{\circ}$ phase imbalance. Therefore, based on the simulation, it is fair to say that in such a case, the interference is Gaussian. 


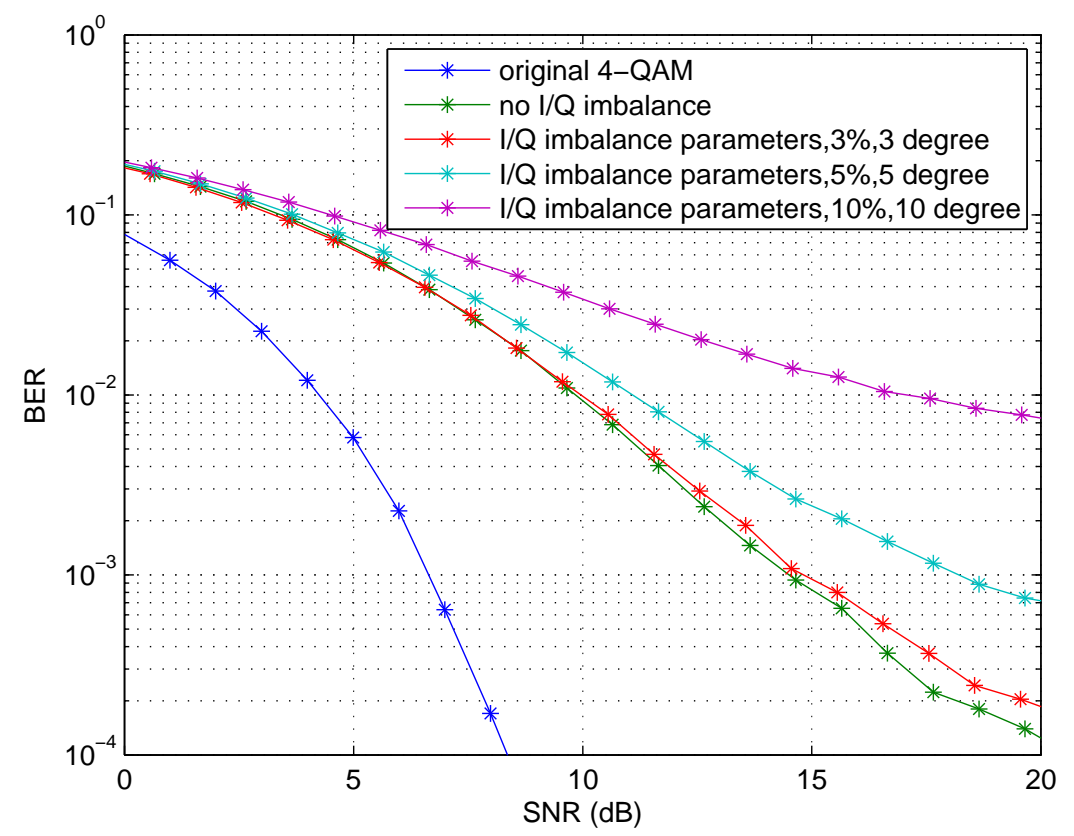

Figure 4.26: BER performance of OFDM with joint effects, different I/Q imbalance parameters, 4-QAM, AWGN Channel

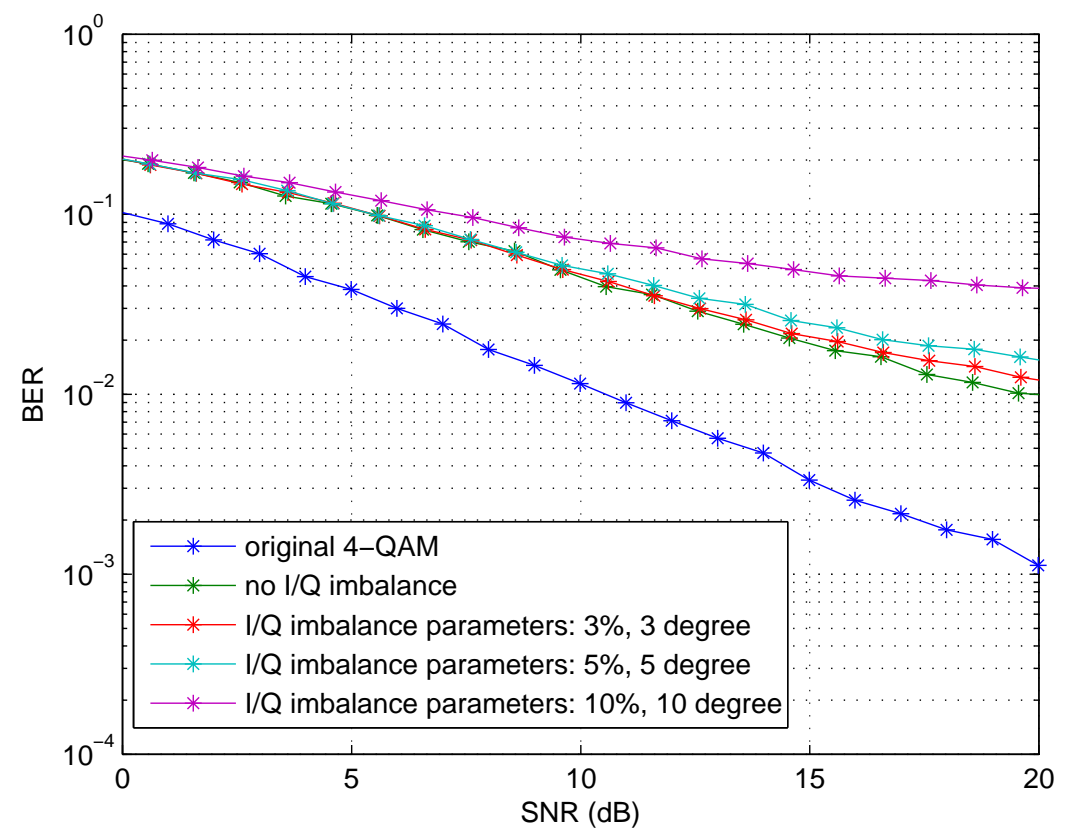

Figure 4.27: BER performance of OFDM with joint effects, different I/Q imbalance parameters, 4-QAM, 4-tap Rayleigh fading Channel 

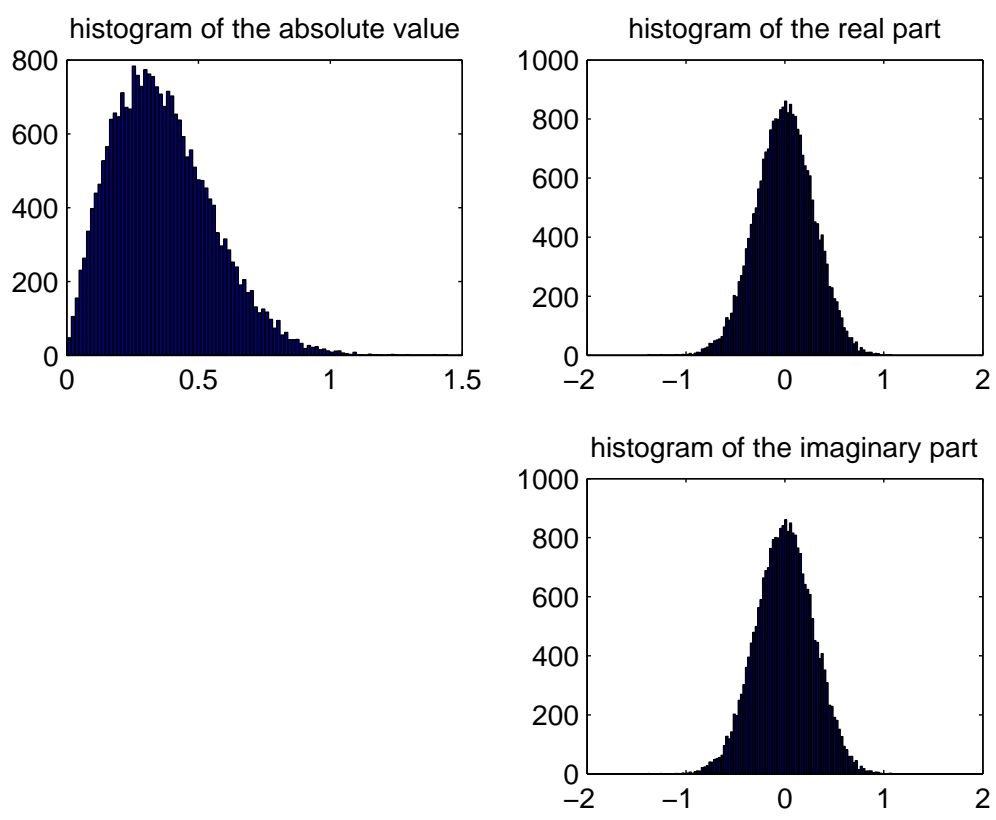

Figure 4.28: Frequency domain histograms of interferences caused by joint effects, $\triangle \varphi_{t}=\triangle \varphi_{r}=1^{\circ}, \gamma_{t}=\gamma_{r}=0.01$
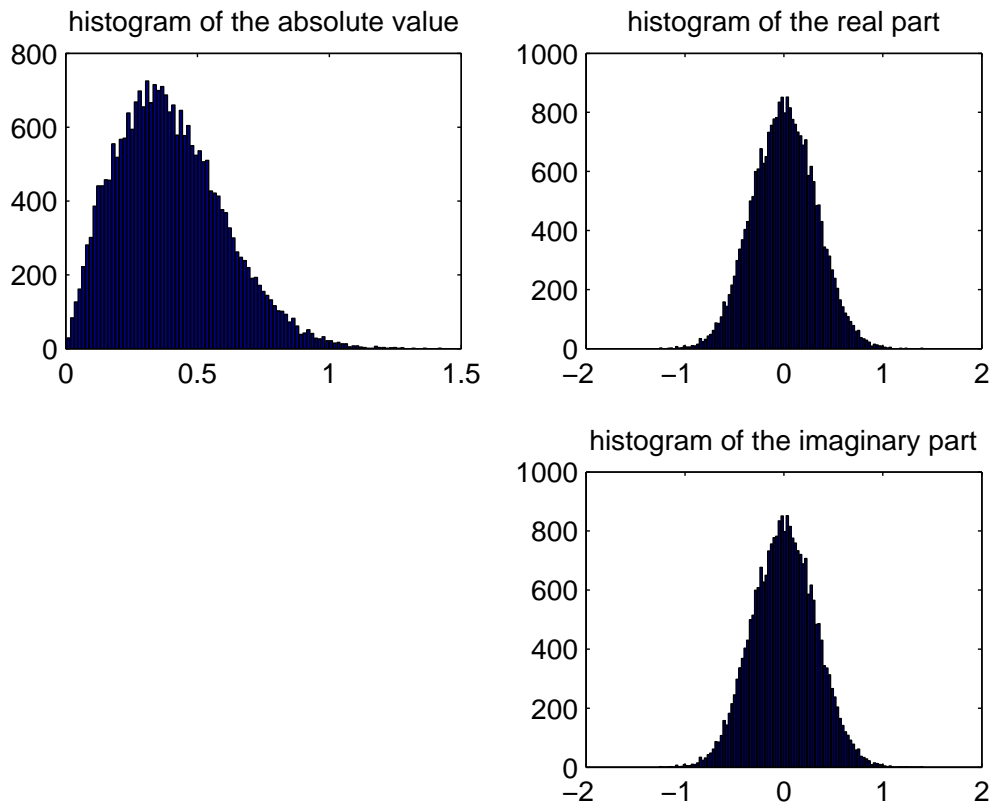

Figure 4.29: Frequency domain histograms of interferences caused by joint effects, $\triangle \varphi_{t}=\triangle \varphi_{r}=3^{\circ}, \gamma_{t}=\gamma_{r}=0.03$ 

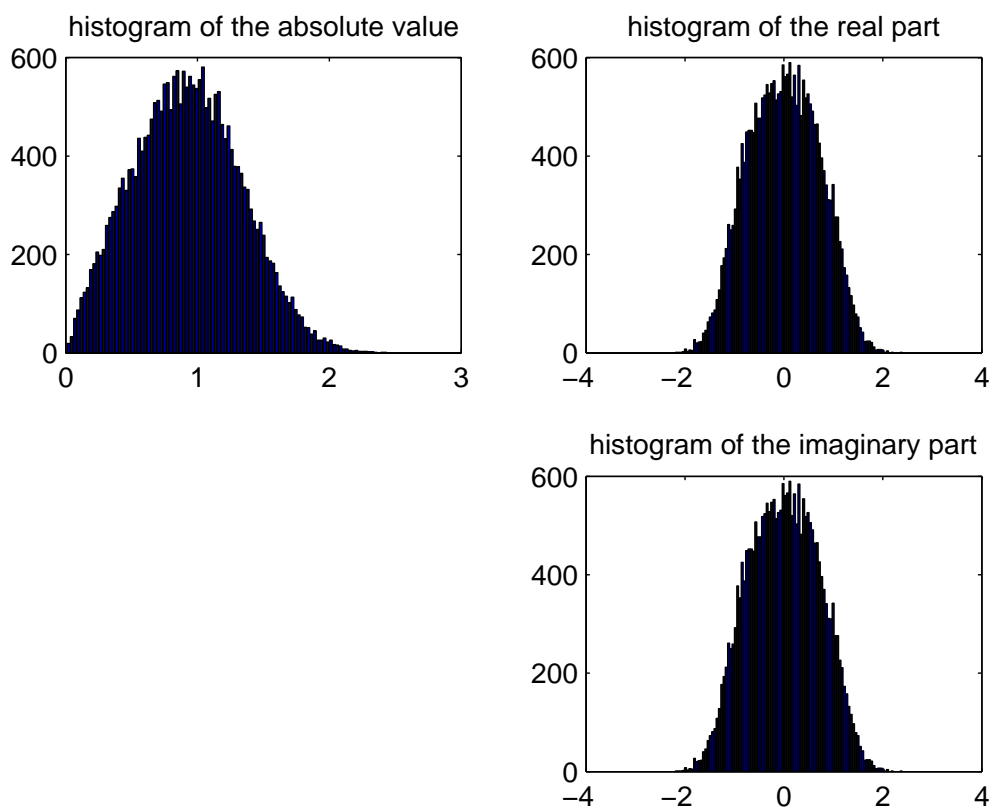

Figure 4.30: Frequency domain histograms of interferences caused by joint effects, $\triangle \varphi_{t}=\triangle \varphi_{r}=10^{\circ}, \gamma_{t}=\gamma_{r}=0.10$ 
If the parameters of I/Q imbalance and nonlinear PA are set to be constant and only the variance of the phase noise is changed, we could see the effects of phase noise in the joint effects. The parameters are $\triangle \varphi_{t}=\Delta \varphi_{r}=5^{\circ}, \gamma_{t}=\gamma_{r}=0.05$ for I/Q imbalance, and SSPA model is used with $\mathrm{OBO}=4.2 \mathrm{~dB}$ for 4-QAM and $\mathrm{OBO}=7.4 \mathrm{~dB}$ for 16-QAM. The BER performances of OFDM system with different variances of phase noise in AWGN channel are presented in the Figures 4.31 and 4.33. In Figure 4.32, the BER performance of OFDM in Rayleigh fading channel is presented.

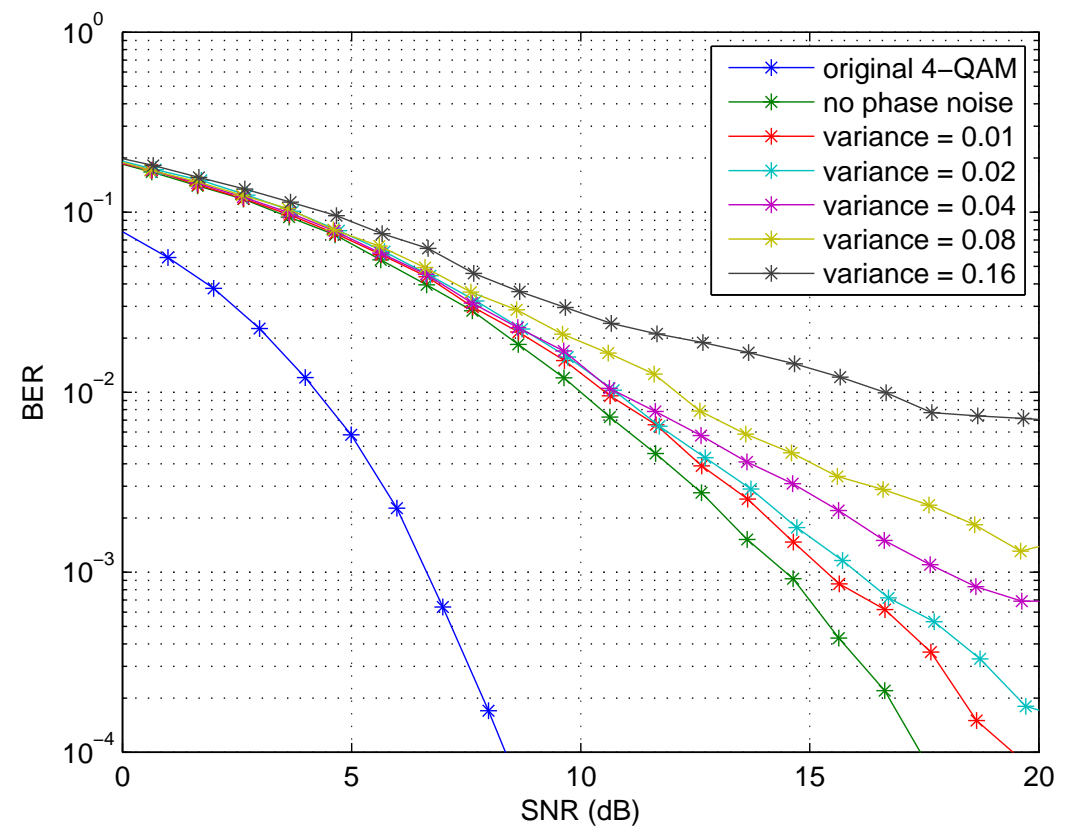

Figure 4.31: BER performance of OFDM with joint effects, different variance of phase noise, 4-QAM, AWGN channel

Different curves in each figure correspond to different values of variance of phase noise. It is apparent that the performance degrades strongly for variance $>0.04$ in Figure 4.31. For the case of 16-QAM in Figure 4.33, it can be seen that even without phase noise and with a high OBO value, the BER performance is strongly degraded by the other two impairments. The BER curve for variance $=0.01$ and the curve for no phase noise are nearly the same, which means that when the variance is very small, the impairment for the system performance is slight. Compared to 4-QAM, there is no such gap between BER curves of different variance. For the case of Rayleigh fading channel, the 


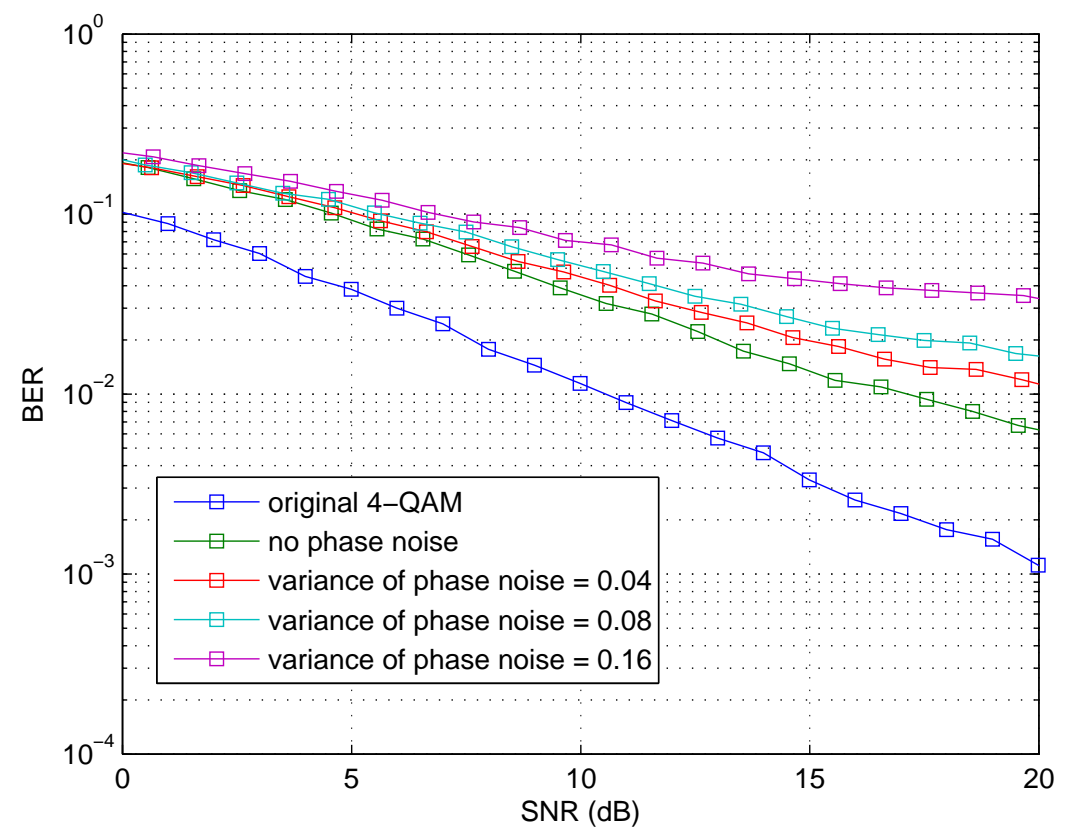

Figure 4.32: BER performance of OFDM with joint effects, different variance of phase noise, 4-QAM, 4-tap Rayleigh fading Channel

effect of phase noise is nearly the same as the case of AWGN channel.

Figures 4.34-4.37 show the frequency domain PDF histograms of the interference caused by joint effects when different variances of phase noise are used. From these four figures, we could see in the frequency domain the PDF follows the Gaussian distribution for the used combination of the impairment parameters. 


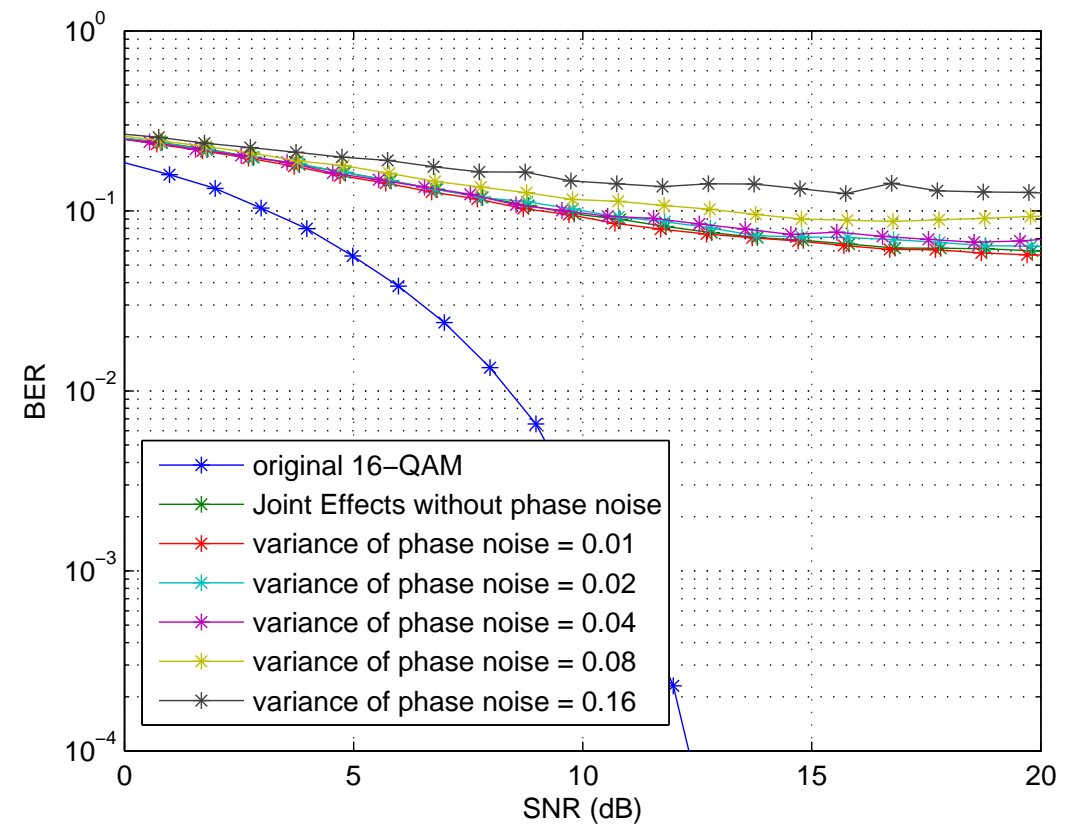

Figure 4.33: BER performance of OFDM with joint effects, different variance of phase noise, 16-QAM, AWGN channel
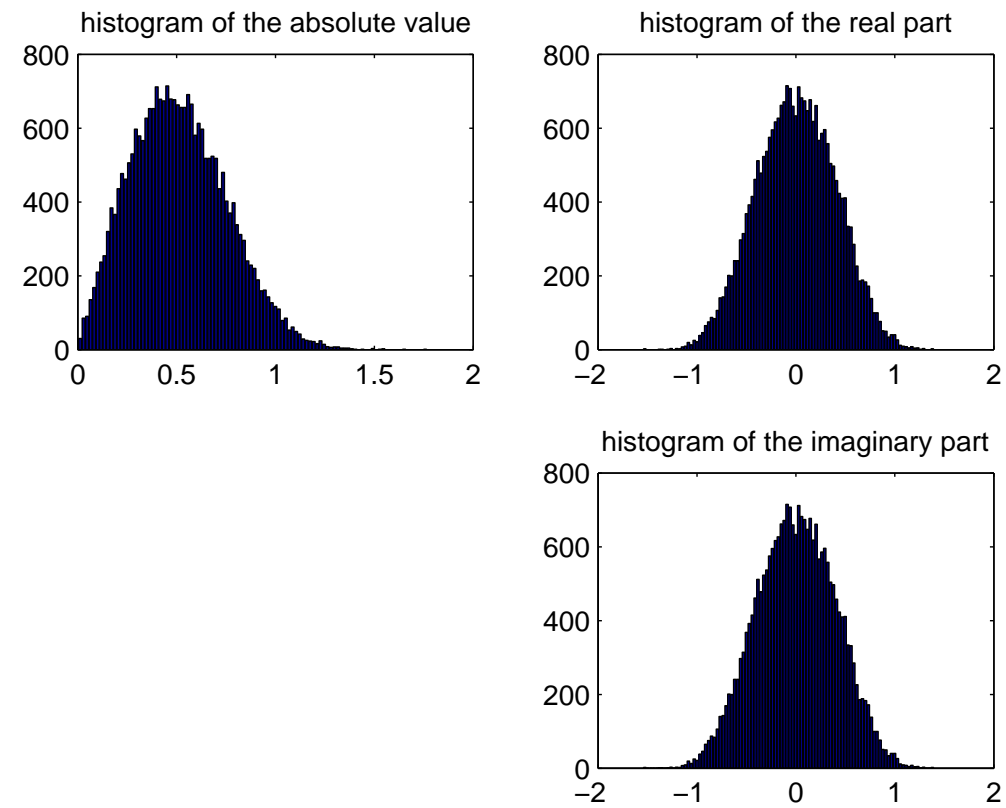

Figure 4.34: Frequency domain histograms of interferences caused by joint effects, no phase noise 

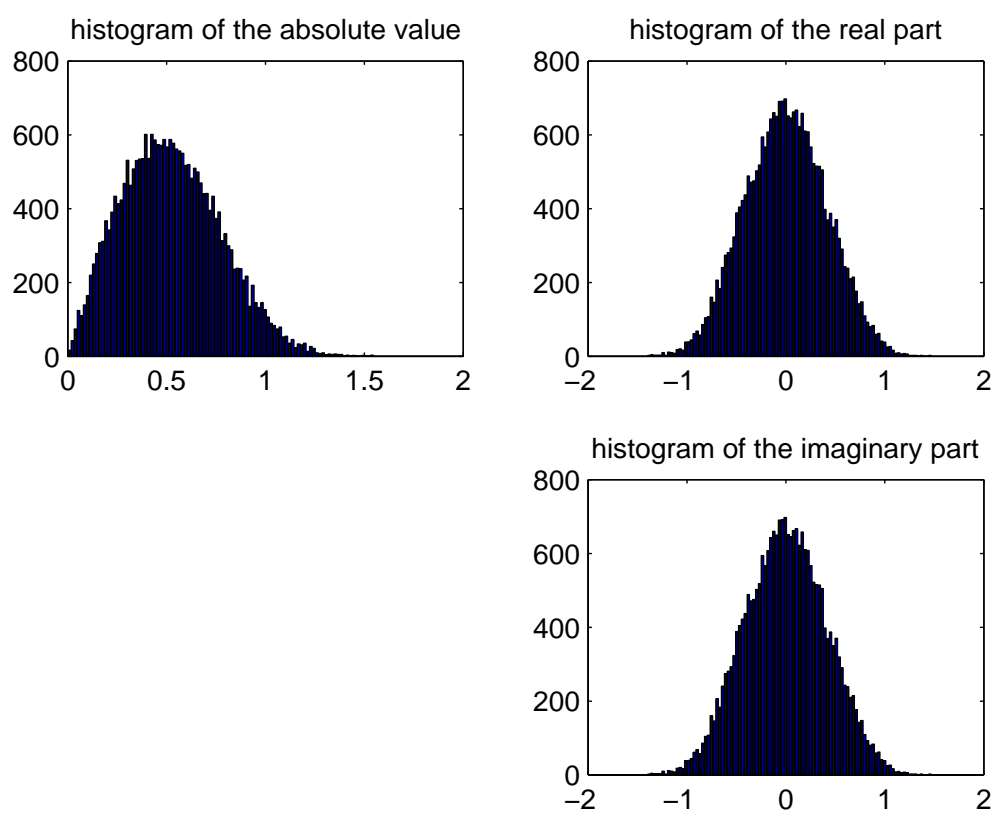

Figure 4.35: Frequency domain histograms of interferences caused by joint effects, variance of phase noise $=0.04$
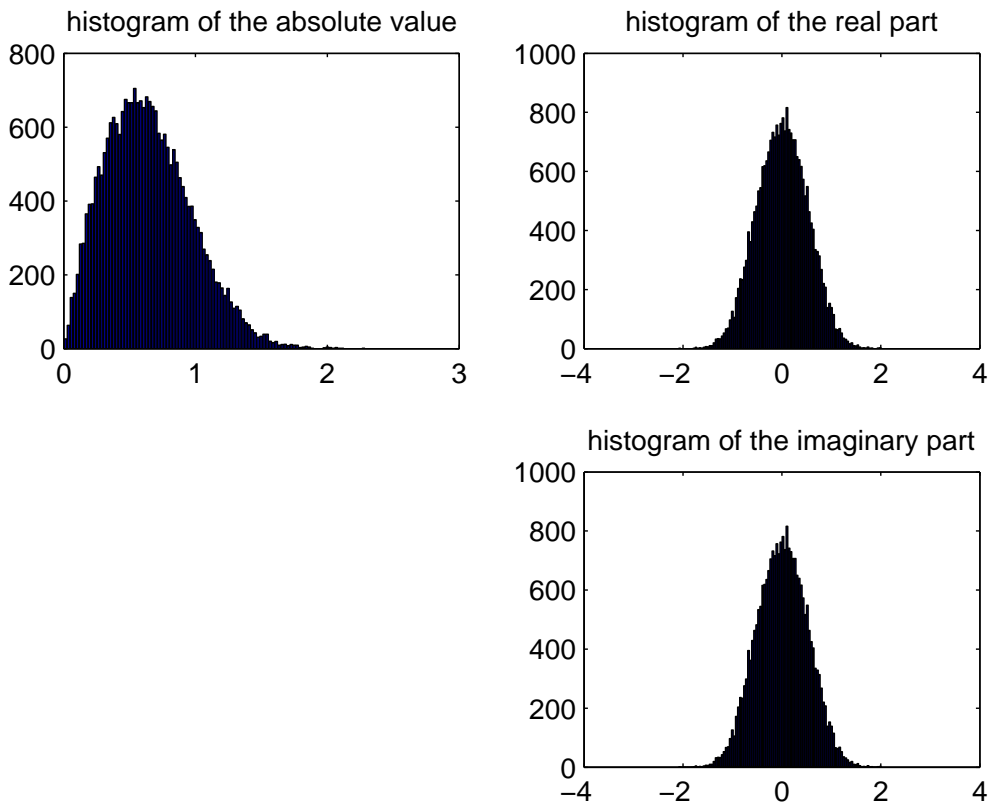

Figure 4.36: Frequency domain histograms of interferences caused by joint effects, variance of phase noise $=0.16$ 

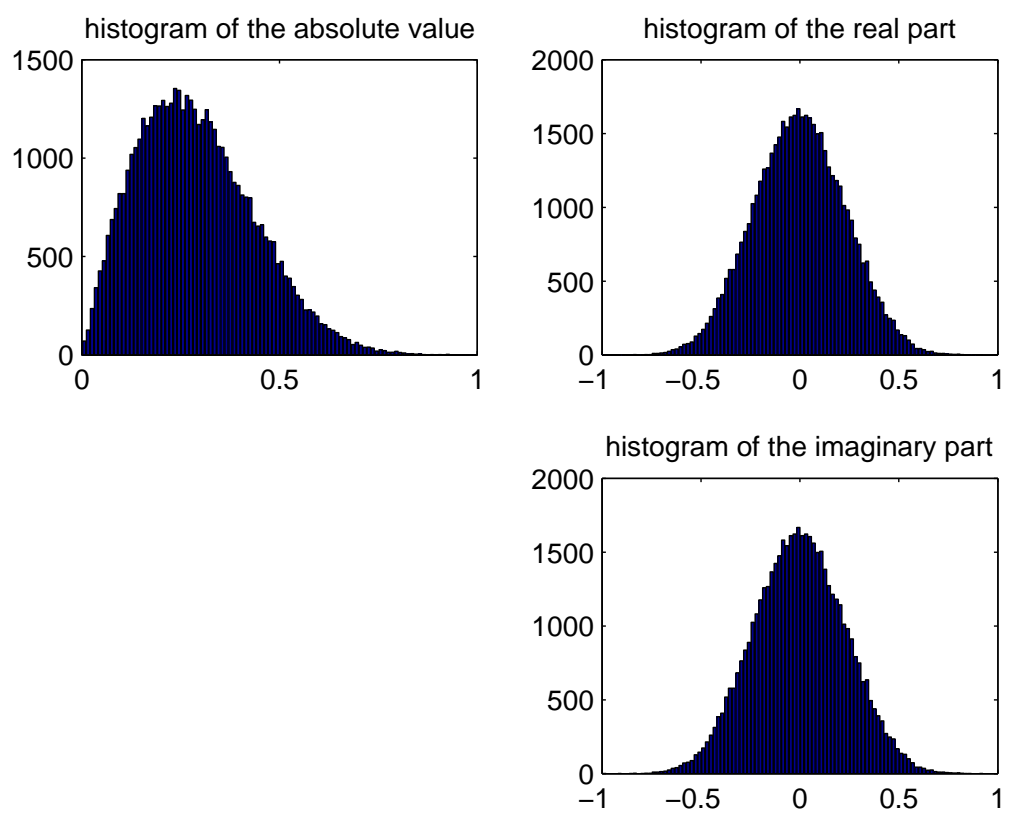

Figure 4.37: Frequency domain histograms of interferences caused by joint effects, variance of phase noise $=0.6$ 
If the parameters of the PA are changed, it is possible to show how nonlinear $\mathrm{PA}$ affects the system in the joint effects. Figure 4.38 presents the BER performance when SSPA is used with different OBO values. The parameters of $\mathrm{I} / \mathrm{Q}$ imbalance are $\Delta \varphi_{t}=\Delta \varphi_{r}=5^{\circ}, \gamma_{t}=\gamma_{r}=0.05$ and variance of phase noise is 0.04. From Figure 4.38, we can see that the nonlinear effects of PA seriously degrades the performance of OFDM transmission especially when OBO is getting smaller. For example, when $\mathrm{BER}=10^{-3}$, the degradation is around $5 \mathrm{~dB}$ from when $\mathrm{OBO}=5.6 \mathrm{~dB}$ to when $\mathrm{OBO}=4.2 \mathrm{~dB}$. Figure 4.39 shows the the BER performance when TWTA is used with different OBO values. The same phenomenon is shown in Figure 4.39 when $\mathrm{OBO}<4.8 \mathrm{~dB}$.

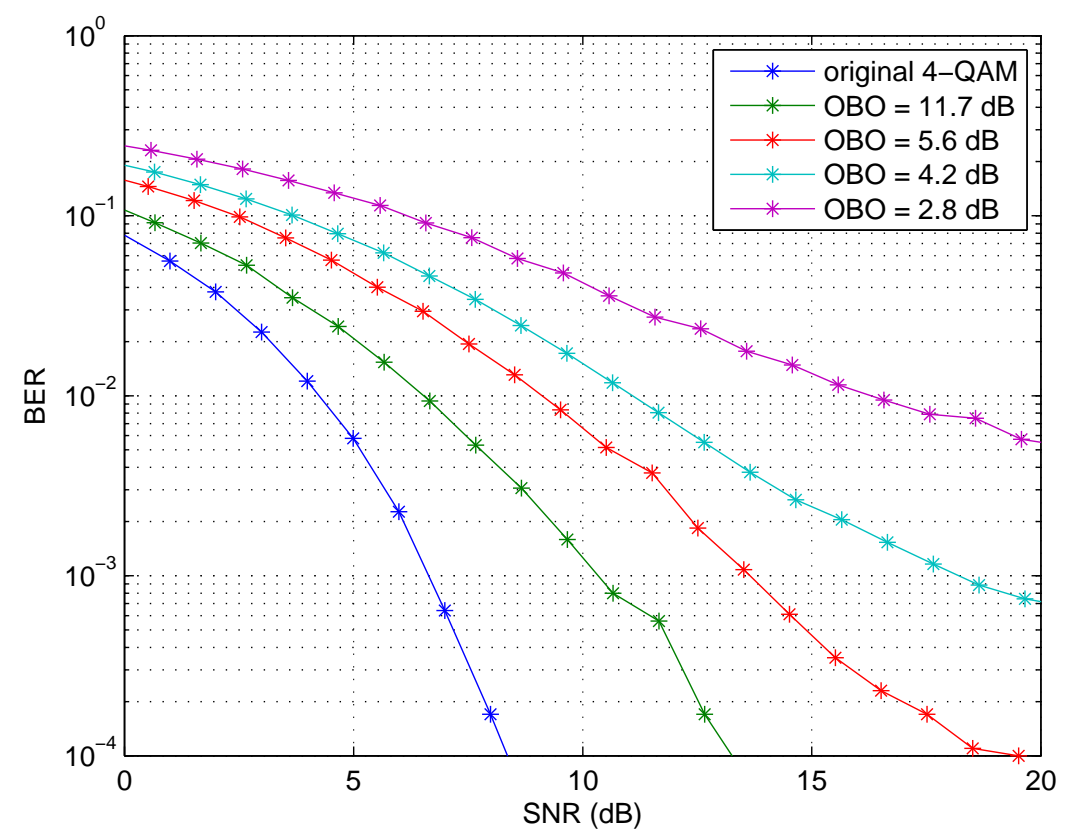

Figure 4.38: BER performance of OFDM with joint effects, different OBO of SSPA, 4-QAM, AWGN channel

In Figures 4.40 and 4.41, BER performances are presented when SSPA and TWTA are used. The different OBO values are presented. The channel model is a 4-tap Rayleigh fading channel. Figure 4.40 shows that when $\mathrm{OBO}<5.6$ $\mathrm{dB}$. the degradation is almost the same when $\mathrm{OBO}$ value gradually decreases. However, the degradation when OBO decreases from $11.7 \mathrm{~dB}$ to $5.6 \mathrm{~dB}$ is the same as that when $\mathrm{OBO}$ decreases from $5.6 \mathrm{~dB}$ to $4.3 \mathrm{~dB}$. The same situation can be found in Figure 4.41. However, the degradation when OBO decreases 


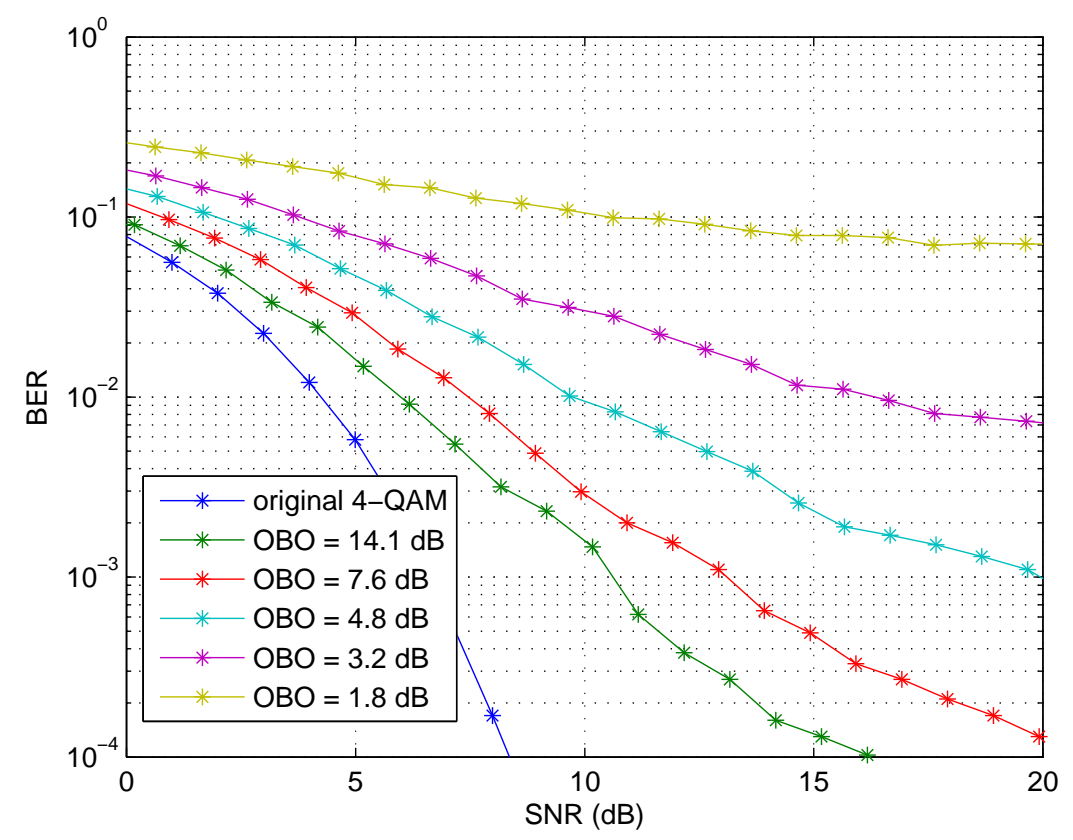

Figure 4.39: BER performance of OFDM with joint effects, different OBO of TWTA, 4-QAM, AWGN channel

from $4.7 \mathrm{~dB}$ to $3.3 \mathrm{~dB}$ is bigger than others.

Figures 4.42 and 4.43 show the frequency domain PDF histograms of noise caused by joint effects with different $\mathrm{OBO}$ value. It can be seen that when OBO value is small, the frequency domain interference can be assumed to be Gaussian. However, when OBO value is larger, the assumption can not be held any more. This is because when OBO is larger, the distribution of NLD differs from Gaussian. 


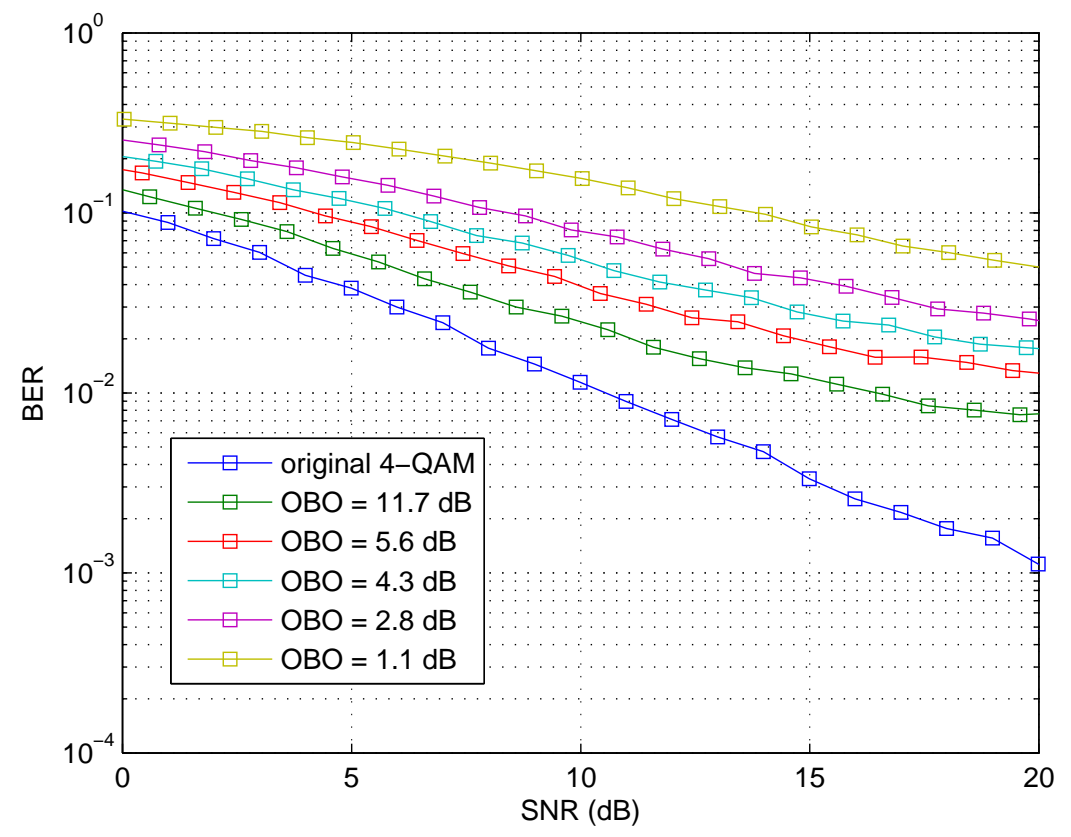

Figure 4.40: BER performance of OFDM with joint effects, different OBO of SSPA, 4-QAM, 4-tap Rayleigh fading channel

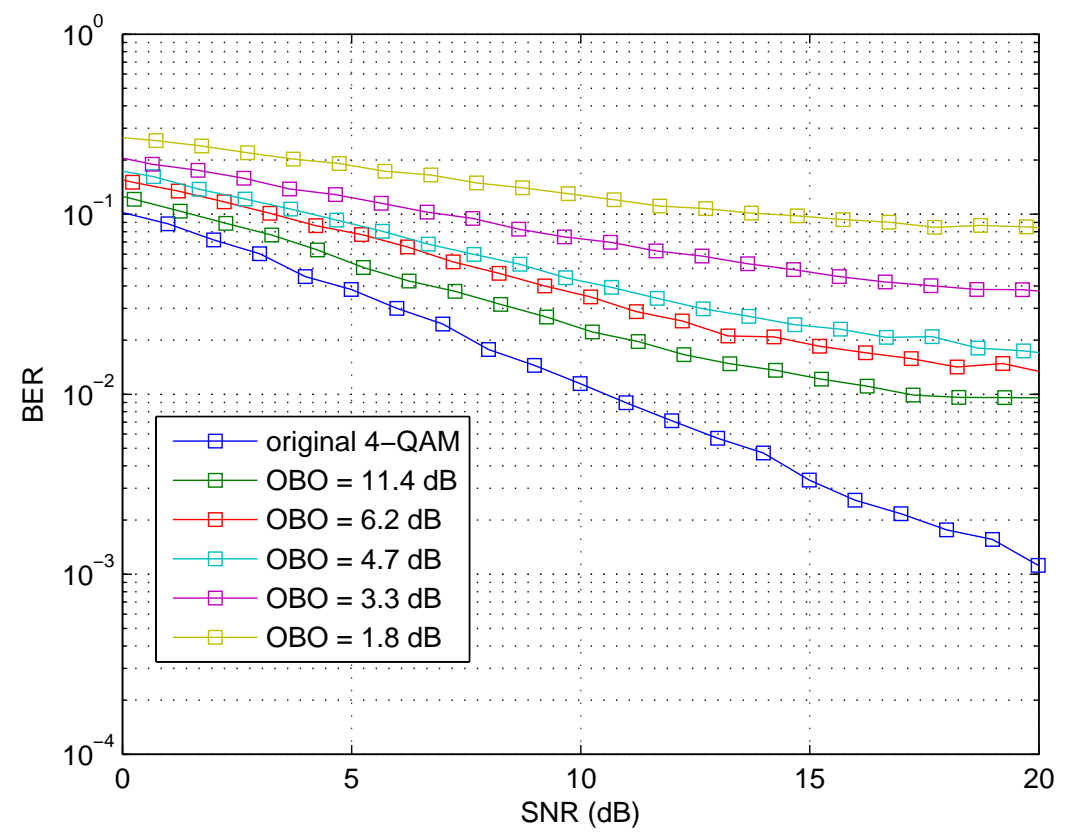

Figure 4.41: BER performance of OFDM with joint effects, different OBO of TWTA, 4-QAM, 4-tap Rayleigh fading channel 

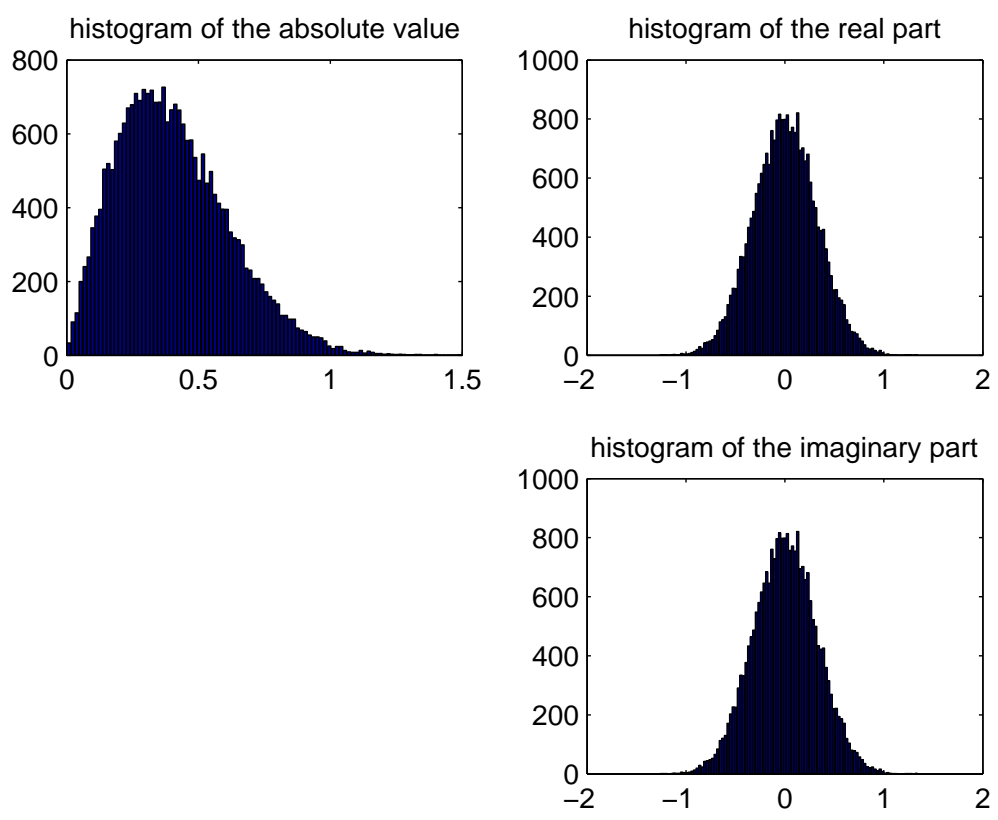

Figure 4.42: Frequency domain histograms of interferences caused by joint effects, SSPA, OBO = $1.6 \mathrm{~dB}$,
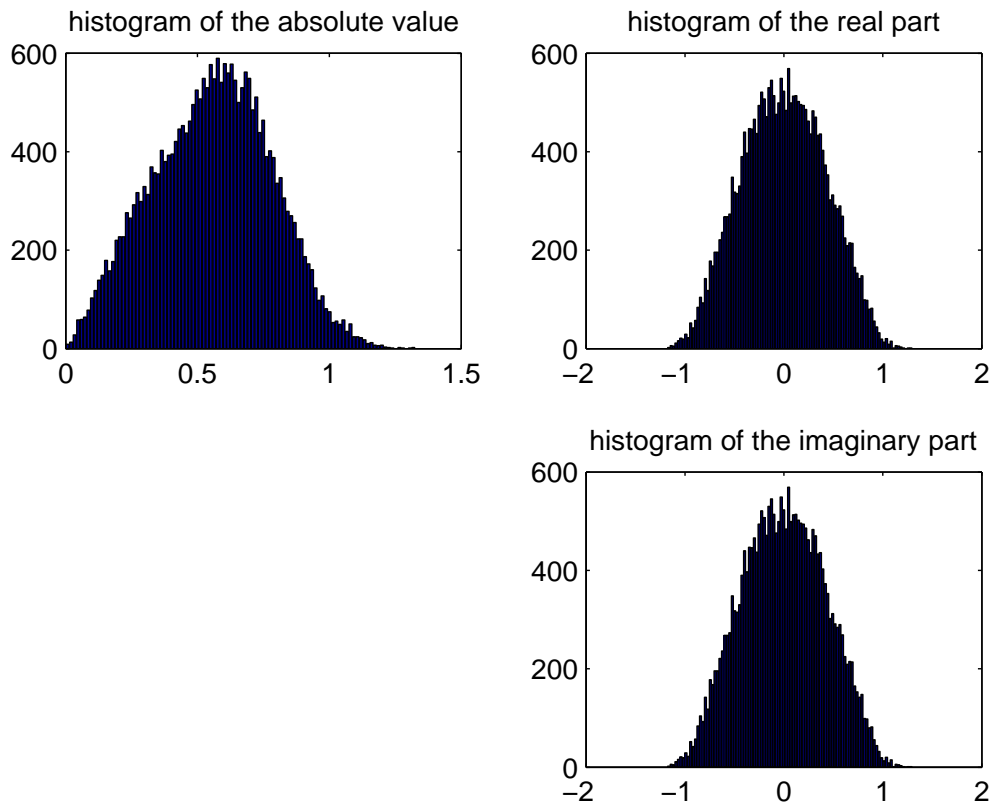

Figure 4.43: Frequency domain histograms of interferences caused by joint effects, SSPA, OBO = $10.6 \mathrm{~dB}$, 


\subsection{Discussion and Summary}

The presumption and theory of dirty RF presented in Chapter 3 are that the RF impairments degrade the performance of OFDM. According to some literatures, for instance, [17], [22] and [37], the statistical properties of interference caused by RF impairment could be generally concluded.

In simulations, we observed BER curves with different RF impairment parameters. Obviously, it can be noticed that the joint effects of dirty RF results in degradation larger than that caused by single one RF impairment. All the simulation results of individual RF impairment are also the basis of the analysis of joint effects.

From the figures in Section 4.2.2, the degradation of $\mathrm{I} / \mathrm{Q}$ imbalance on the BER performance is becoming a serious problem as number of symbol alphabet increases. For each I/Q set of imbalance parameters used in this thesis, the degradation of performance of the OFDM system changes rapidly as the value of I/Q imbalance parameters become larger. For example, in Figure 4.6, when $\mathrm{BER}=10^{-4}$, the degradation is less than $0.5 \mathrm{~dB}$ between no imbalance and $1^{\circ}$ phase imbalance existed. However, the degradation is around $1.5 \mathrm{~dB}$ when the value of phase imbalance changes from $1^{\circ}$ to $3^{\circ}$. When the phase imbalance changes from $3^{\circ}$ to $5^{\circ}$, the degradation becomes more than $3 \mathrm{~dB}$. The PDF histograms of the interference caused by I/Q imbalance do not follow Gaussian distribution in the frequency domain.

In Section 4.2.3, obviously we could see that for different values of variance of phase noise, BER for 4-QAM does not degrade so noticeable compared to the ones for 16-QAM and 64-QAM, which means higher order modulation suffer more from the effects of phase noise. Figure 4.13 shows that for 16-QAM even though the variance of phase noise $=0.02$, the degradation is still severe. When the variance of phase noise is small, the interference can be seen as approximately Gaussian. However as the variance increases, the assumption cannot be held.

The BER performance of the tested SSPA is somewhat worse than that of the tested TWTA for the same OBO value, which results from the value of $K_{o}$ 
being smaller after the SSPA than after the TWTA even if there is less noise power after the SSPA. For the OFDM system with SSPA, the performance is severe degraded when $\mathrm{OBO}<4.2 \mathrm{~dB}$ in the simulation. However, for the one with the TWTA, the same situation happens when $\mathrm{OBO}<3.4 \mathrm{~dB}$. Nonlinear noise that is caused by a PA is Gaussian-like for relatively small OBO values no matter what kind of PA is used.

When three RF impairments are considered together, it is observed that the OFDM system is degraded much worse than that when considering one single impairment. In this work, we have analyzed both joint and individual effect of impairments. Furthermore the histograms of interference caused by different parameters are presented. This information can be used for a future research since more work can be done if it is possible derive and conclude the properties of interference. 


\section{Chapter 5}

\section{Conclusions and Future Work}

This chapter concludes the main results achieved in this thesis. And most importantly, some possible future research directions based on this thesis are discussed as well.

\subsection{Conclusions}

OFDM transmission has many favorable features, such as robustness against multipath fading and narrow-band interference, high spectral efficiency and simple channel estimation and equalization, which are why it is an attractive method for wireless communication systems. One very important problem of OFDM is its sensitivity to the RF impairments.

This thesis gives a basic overview of OFDM modulation method and discusses the advantages and disadvantages in Chapter 2. In Chapter 3, three RF impairments that degrade the performance of OFDM system are introduced in details. The basic of phase noise are presented in Section 3.1, and the main effects of phase noise as well as some compensation methods are described. The models and compensation methods of $\mathrm{I} / \mathrm{Q}$ imbalance are presented in Section 3.2. Three frequently used models of memoryless PA are introduced. Different reduction techniques for PA nonlinearity have been briefly presented. In addition, two other widely known RF impairments, jitter and CFO, are briefly introduced in Chapter 3. Some formulas are derived to understand the joint effects of dirty RF in Chapter 4. Simulation results can demonstrate how these impairments limit the performance of systems. Following discussions are made 
to illustrate the joint effects.

\subsection{Possible Future Work}

One interesting research area for the joint effects of dirty RF is to analyze the statistical property of the interference introduced by all three RF impairments. It is useful for deriving the theoretical expression of the BER of OFDM systems with dirty RF.

Another important research direction is to derive compensation algorithms for the joint effects. One possible way to accomplish this is to combine the regular algorithms for individual RF impairments together. However, considering the complexity of such an algorithm, it is perhaps better to find more suitable way to compensate for all the RF impairments together. 


\section{Bibliography}

[1] ETSI, Radio Broadcasting Systems; Digital Audio Broadcasting (DAB) to mobile, portable and fixed receivers, EN 300401 V1.3.3, May 2001.

[2] ETSI, Digital Video Broadcasting (DVB); framing structure, channel coding and modulation for digital terrestrial television, EN 300744 V1.5.1, November 2004.

[3] ITU, Asymmetrical Digital Subscriber Line (ADSL) Transceivers: Transmission media and System, G.992.1, July 1998.

[4] IEEE, Wireless LAN Medium Access control (MAC) and physical layer (PHY) specifications: High Speed Physical Layer in the 5 Ghz Band, IEEE Std 802.11a-1999.

[5] IEEE, Wireless LAN Medium Access Control (MAC) and Physical Layer (PHY) specifications. Further Higher-Speed Physical Layer Ex- tension in the 2.4 GHz Band, IEEE Std 802.11g-2003.

[6] ETSI, Broadband radio access networks (BRAN); HIPERLAN type 2; Physical (PHY) layer, TS 101475 V1.1.1, 2000-2004.

[7] E. Costa, M. Midrio and S. Pupolin, "Impact of Amplifier Nonlinearities on OFDM Transmission System Performance," IEEE Communication Letter, vol.3, no.2, February 1999.

[8] L. Litwin and M. Pugel, "The principle of OFDM", Thomson Multimedia, January 2001.

[9] R. Prasad, OFDM for wireless communications systems, Artech House, 2004.

[10] R. Van Nee and R. Prasad, OFDM for wireless multimedia communications, Artech House,2000. 
[11] D. Petrovic, W. Rave and G. Fettweis, "Effects of Phase Noise on OFDM systems With and Without PLL: Characterization and Compensation," IEEE Trans. Communications, vol.55, no.5, Auguest 2007.

[12] L. Tomba, "On the Effect of Wiener Phase Noise in OFDM systems," IEEE Trans. Communications, vol.46, no.5, May 1998.

[13] A. Armada, "Understanding the Effects of Phase Noise in Orthogonal Frequency Division Multiplexing (OFDM)," IEEE Trans. Broadcasting, vol.47, no.2, June 2001.

[14] D. Petrovic, W. Rave and G. Fettweis, "Common Phase Error due to Phase Noise in OFDM - Estimation and Suppression," IEEE Proc.PIMRC, September 2004.

[15] S. Wu and Y. Bar-Ness, "OFDM Systems in the Presence of Phase Noise: Consequences and Solutions," IEEE Trans. Communications, vol.52, no.11, November 2004.

[16] A. Tarighat and A. Sayed, "Joint Compensation of Transmitter and Receiver Impairment in OFDM systems," IEEE Trans. Wireless Communications, vol.6, no.1, January 2007.

[17] C. Liu, "Impacts of I/Q imbalance on QPSK-OFDM-QAM detection," IEEE Trans. Consumer Electronic, vol.44, no.3, August 1998.

[18] D. Tandur and M. Moonen, "Joint Adaptive Compensation of Transmitter and Receiver IQ Imbalance Under Carrier Frequency Offset in OFDMBased Systems," IEEE Trans. Signal Processing, vol.55, no.11, November 2007.

[19] J. Tubbax, B. Come, L. Van der Perre, S. Donnay, M. Moonen and H. De Man, "Compensation of Transmitter IQ Imbalance for OFDM Systems," IEEE International Conference on Acoustics, Speech, and Signal Processing(ICASSP), May 2004.

[20] A. Tarighat, R. Baghari, and A. Sayed, "Compensation Schemes and Performance Analysis of IQ imbalances in OFDM Receiver," IEEE Trans. Signal Processing, vol.53, no.8, August 2005.

[21] S. Cripps, RF Power Amplifier for Wireless Communication, Artech House, 2002. 
[22] D. Dardari,V. Tralli and A. Vaccari, "A Theoretical Characterization of Nonlinear Distortion Effects in OFDM Systems," IEEE Trans. Communications, vol.48, no.10, October 2000.

[23] C. Rapp, "Effects of HPA-Nonlinearity on a 4-DPSK/OFDM-Signal for a Digital Sound Broadcasting System," In Proc. of the second European Conference on Satellite Communication, October 1991.

[24] A. A. M. Saleh, "Frequency-Independent and Frequency-Dependent Nonlinear Models of TWT Amplifier," IEEE Trans. Communications, vol.29, no.11, November 1981.

[25] G. Santella and F. Mazzenga, "A hybrid analytical-simulation procedure for performance evaluation in M-QAM-OFDM schemes in presence of nonlinear distortion," IEEE Trans. Vehicular Technologies, vol.47, no.1, February 1998.

[26] D. Dardari, V. Tralli and A. Vaccari, "Analytical Evaluation of total Degradation for OFDM system with TWTA or SSPA," CSITE Tech..

[27] P. B. Kenington, High-Linearity RF Amplifier Design, Artech House,2000.

[28] M. Di Benedetto and P. Mandarini, "An Application of MMSE Predistortion to OFDM Systems," IEEE Trans. Communications, vol.44, no.11, November 1996.

[29] Y. Ding, Y. Liu, I. Nilkhamhang and A. Sano, "Adaptive Linearization for Power Amplifier in OFDM Systems," SICE-ICASE, October 2006.

[30] F.H. Gregorio, S. Werner, J.E. Causseau and R. Wichman ,"Broadband power amplifier distortion cancellation with model estimation in the receiver," IEEE Workshop on Signal Processing Advances in W ireless Communications (SPAWC), 2007.

[31] M. Lohning, D. Petrovic, M. Windisch, P. Zillmann and W. Rave, "Dirty RF: a new paradigm," IEEE 16th International Symposium on Personal, Indoor and Mobile Radio Communications(PIMRC), 2005.

[32] K. Sathananthan and C. Tellambura, "Probability of Error Calculation of OFDM Systems With Frequency Offset," IEEE Trans. Communications, vol. 49, no.11, November 2001. 
[33] J. Tubbax, B. Come, L. Van der Perre, S. Donnay, M. Engels, H. De Man and M. Moonen, "Compensation of IQ Imbalance and Phase Noise in OFDM Systems," IEEE Trans. Wireless Communications, vol.4, no.3, May 2005.

[34] Q. Zou, A. Tarighat and A. Sayed, "Joint Compensation of IQ Imbalance and Phase Noise in OFDM Systems," Fortieth Asilomar Conference on Signals, Systems and Computers (ACSSC), 2006.

[35] C. Tsai, C. Liao, and T. Chiueh, "IQ Imbalance and Phase Noise Mitigation for Wireless OFDM systems," IEEE International Symposium on Circuits and Systems (ISCAS), 2008.

[36] E. Costa and S. Pupolin, "M-QAM-OFDM Systems Performance in the Presence of a Nonlinear Amplifier and Phase Noise," IEEE Trans. Communications, vol.50, no.3, March 2002.

[37] P. Liuăand Y. Bar-Ness, "Closed-Form Expressions for BER Performance in OFDM Systems with Phase Noise," IEEE International Conference on Communications, 2006. 


\section{Appendix}

\section{$\%$}

Zheng Chang 2008

Simulator for the performance analysis of OFDM system with dirty RF. The RF impairments include I/Q imbalance at the transmitter and receiver, phase noise at the receiver and nonlinear memoryless PA. No channel effect, AWGN channel and Rayleigh fading channel are used.

$\begin{array}{ll}\text { \% } & \\ \text { qam } & \text { :QAM modulation } \\ \text { ofdm } & \text { OFDM modulation } \\ \text { iqimbalance } & \text { :generete I/Q imbalance } \\ \text { pa } & : \text { PA model } \\ \text { awgn } & : \text { MATLAB function, AWGN channel } \\ \text { rayleighfading } & : \text { Rayleigh fading channel } \\ \text { phasenoise } & : \text { generate phase noise } \\ \text { de_ofdm } & : \text { demodulate OFDM signal } \\ \text { symerr } & : \text { MATLAB function, get BER } \\ \%\end{array}$


close all

clear all

clc

$\begin{array}{ll}\% & \\ \% & \text { Initialization } \\ \%- & \\ \text { drops }=1000 ; & \% \text { Number of independent runs. } \\ \mathrm{M}=16 ; & \% \text { M-QAM modulation. }\end{array}$

Number_of_subcarriers $=64 ; \%$ Number of the OFDM subcarrier

Length_of_CP $=16 ; \quad \%$ the CP length

Number_of_bits $=10000 ; \quad \%$ Number of bits

$\mathrm{g}_{-} \mathrm{t}=0.05 ; \quad \% \mathrm{I} / \mathrm{Q}$ imbalance factor of amplitude at $\mathrm{Tx}$

fi_t $=$ pi/36; $\quad \% \mathrm{I} / \mathrm{Q}$ imbalance factor of phase at $\mathrm{Tx}$

$\mathrm{g} \_\mathrm{r}=0.05 ; \quad \% \mathrm{I} / \mathrm{Q}$ imbalance factor of amplitude at $\mathrm{Rx}$

fi_r $=$ pi $/ 36 ; \quad \% \mathrm{I} / \mathrm{Q}$ imbalance factor of phase at $\mathrm{Rx}$

SNR_dB $=-5: 35 ; \quad \%$ channel SNR

A_sat $=3 ; \quad \%$ input saturation voltage

$\mathrm{Es}=1$

$\mathrm{T}=4 ; \quad \%$ numbers of channel tap

variance_of_pn $=0.04 ; \quad \%$ variance of the phase noise
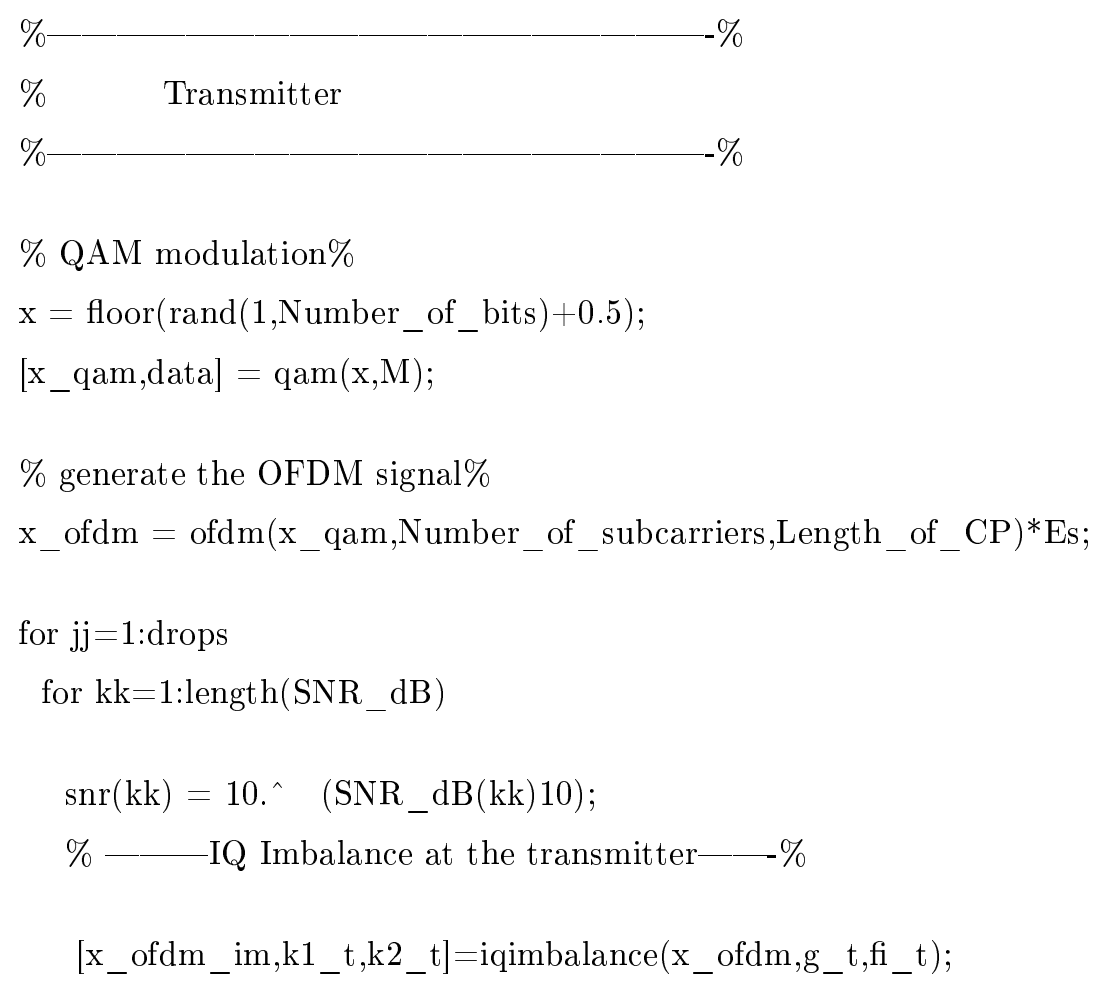


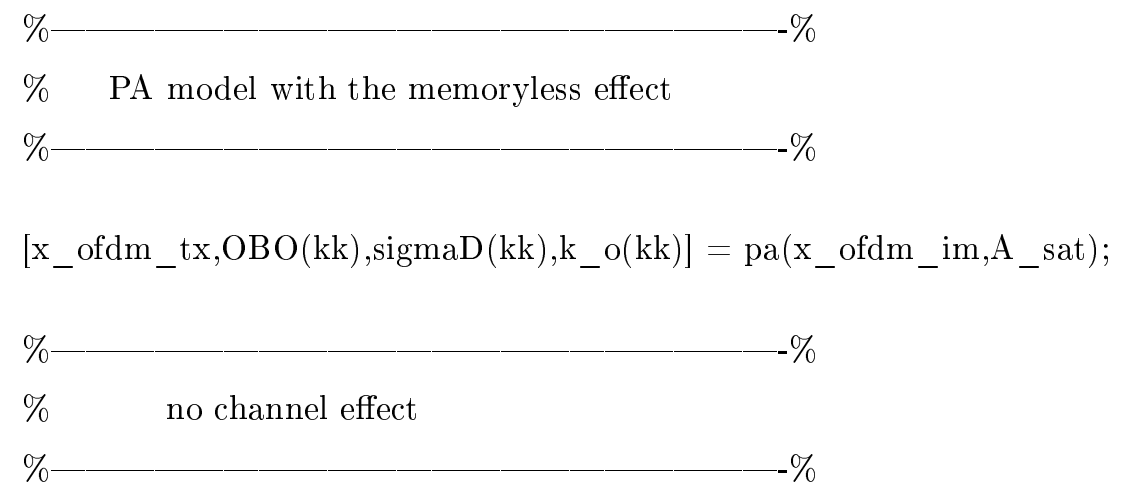

$\%$ channel_output $=\mathrm{x} \_$ofdm_tx; $\operatorname{sigma} W(\mathrm{kk})=0$;

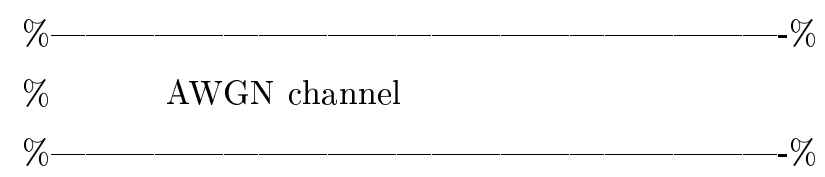

channel_output $=$ awgn $\left(x_{\text {_ }}\right.$ ofdm_tx,SNR_dB(kk),'measured');

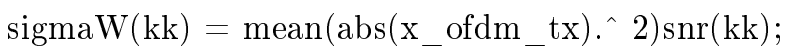

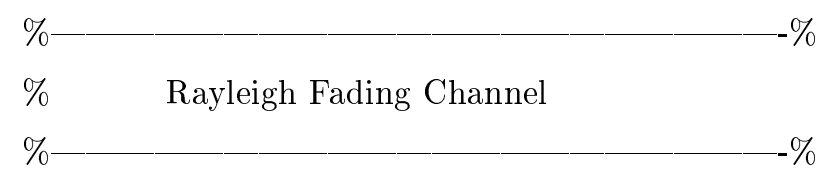

$\%$ [channel_output,h_channel $]=$ rayleighfading $\left(\mathrm{x} \_\right.$ofdm_tx $\left., \mathrm{T}, \mathrm{snr}(\mathrm{kk})\right)$;

$\% \operatorname{sigmaW}(\mathrm{kk})=\operatorname{mean}\left(\operatorname{abs}\left(\mathrm{x} \_ \text {ofdm_tx }\right)^{\wedge} 2\right) \operatorname{snr}(\mathrm{kk})$;

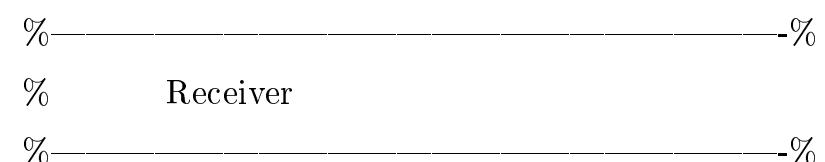

$\%-\mathrm{I} / \mathrm{Q}$ Imbalance at the receiver-—— $\%$

$\left[\mathrm{rx} \_\mathrm{im}, \mathrm{k} 1 \_\mathrm{r}, \mathrm{k} 2 \_\mathrm{r}\right]=\mathrm{iqimbalance}\left(\mathrm{channel} \_\right.$output,g_r,fi_r);

$\%$ - Phase noise_—— $\%$

$\mathrm{N}=$ Number_of_subcarriers;

$\mathrm{L}=$ Length_of_CP;

v_pn = variance_of_pn;

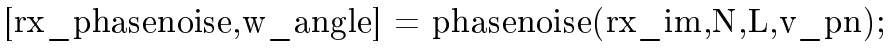

$\mathrm{rx}=\mathrm{rx} \_$phasenoise; 
\% Match filter for Rayleigh fading channel\%

$\% \mathrm{H}=\mathrm{fft}\left(\mathrm{h} \_\right.$channel,length(rx_phasenoise $\left.)\right)$;

$\% \mathrm{rx}=\operatorname{ifft}\left(\mathrm{fft}\left(\mathrm{rx} \_\right.\right.$phasenoise $\left.) . \mathrm{H}\right)$;

$\% \mathrm{rx}=\operatorname{rx}\left(1:\right.$ length $\left(\mathrm{x} \_\right.$ofdm_im $\left.)\right)$;

\%Demodulation of the OFDM signal\%

$\mathrm{z}=$ de_ofdm(rx,Number_of_subcarriers,Length_of_CP $)$;

$\mathrm{z}=\mathrm{z}\left(1:\right.$ length $\left(\mathrm{x}_{-}\right.$qam $\left.)\right)$Es;

$\%$-Evaluation —_— $\%$

$\%$ demodulate the QAM signal\%

$\mathrm{y}=$ qamdemod $(\mathrm{z}, \mathrm{M})$;

$y_{-}$bi $=\operatorname{de} 2 b i(y)$;

y_bi = fliplr(y_bi);

$[\mathrm{m}, \mathrm{n}]=\operatorname{size}($ data $)$;

$y_{-}$final $=[]$;

for $\mathrm{ii}=1: \mathrm{m}$

$\mathrm{y}_{-}$final $=\left[\mathrm{y} \_\right.$final,y_bi $\left.(\mathrm{ii},: \mathrm{:})\right]$;

end

$\%$ The bit error probability \%

$[\operatorname{num}(\mathrm{jj}, \mathrm{kk}), \mathrm{BER}(\mathrm{jj}, \mathrm{kk})]=\operatorname{symerr}\left(\mathrm{y} \_\right.$final, $\mathrm{x}\left(1: \operatorname{length}\left(\mathrm{y} \_\right.\right.$final $\left.\left.)\right)\right) ;$

end

end

ber_whole $=$ mean $(\mathrm{BER})$; 
$\%$ -

function $\left[\mathrm{x} \_\right.$qam,data $]=\mathrm{qam}(\mathrm{x}, \mathrm{M})$

L_QAM $=\log 2(\mathrm{M})$;

for $\mathrm{ii}=1$ :length $(\mathrm{x}) \mathrm{L} \_\mathrm{QAM}$

$\mathrm{x} 1(\mathrm{ii},:)=\mathrm{x}\left((\mathrm{ii}-1) * \mathrm{~L} \_\mathrm{QAM}+1:(\mathrm{ii}-1) * \mathrm{~L} \_\mathrm{QAM}+\mathrm{L} \_\mathrm{QAM}\right)$;

$\mathrm{x} 2(\mathrm{ii},:)=\operatorname{num} 2 \operatorname{str}(\mathrm{x1}(\mathrm{ii},:))$;

end

data $=\operatorname{bin} 2 \operatorname{dec}(x 2)^{\prime}$;

$\mathrm{x} \_\mathrm{qam}=\operatorname{qammod}($ data, $\mathrm{M})$;

$\%$

function $\left[\mathrm{x}_{-}\right.$ofdm $]=\operatorname{ofdm}(\operatorname{data}, \mathrm{N}, \mathrm{L})$

$\% \%$ This is OFDM modulation. CP is inserted.\%\%

c_prefix $=[]$

m_data $=[]$

$\mathrm{T}=\mathrm{N}+\mathrm{L} ; \quad$ \%length of OFDM symbol

$\mathrm{I}=$ length(data);

if $\operatorname{rem}(\mathrm{I}, \mathrm{M})=0$

data $=[$ data,zeros $(1, \mathrm{M}-\operatorname{rem}(\mathrm{I}, \mathrm{N}))]$;

$\mathrm{I}=$ length(data);

end

$\%$ the modulation IFFT \%

for $\mathrm{n}=1: \mathrm{N}: \mathrm{I}-\mathrm{N}+1$

ofdm_temp1(n:n+M-1) $=\operatorname{sqrt}(\mathrm{N}) * \operatorname{ifft}(\operatorname{data}(\mathrm{n}: \mathrm{n}+\mathrm{N}-1), \mathrm{N})$;

temp1 = ofdm_temp1 $(\mathrm{n}: \mathrm{n}+\mathrm{N}-1)$;

$\mathrm{m}_{-}$data $=\left[\mathrm{m} \_\right.$data,zeros $(1, \mathrm{~L})$, temp1 $]$;

end

$\%$ the cyclic prefix $\%$

for $\mathrm{n}=1: \mathrm{N}: \mathrm{I}-\mathrm{N}+1$

ofdm_temp2(n:n+L-1) $=$ ofdm_temp1 $(\mathrm{n}+\mathrm{N}-\mathrm{L}: \mathrm{n}+\mathrm{N}-1)$;

end

ofdm_temp2 $=[$ ofdm_temp2,zeros $(1$, I-length $($ ofdm_temp2 $))]$;

$\%$ inserting cyclic prefix $\%$ 


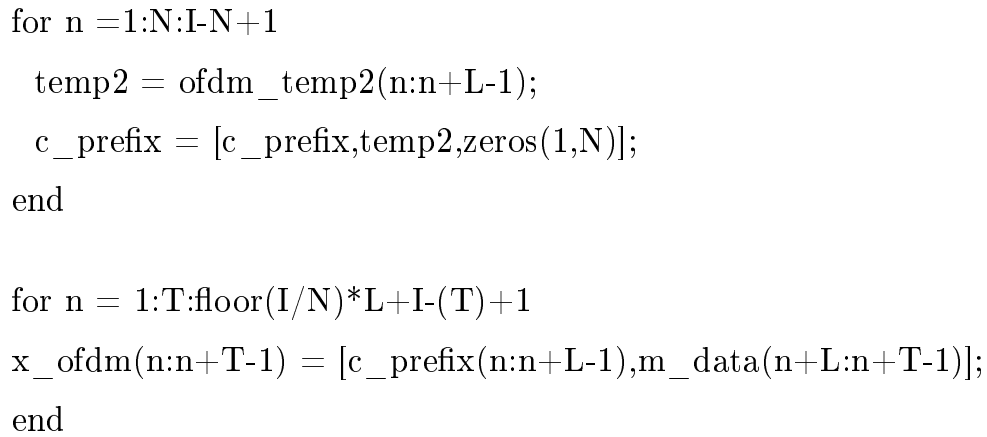




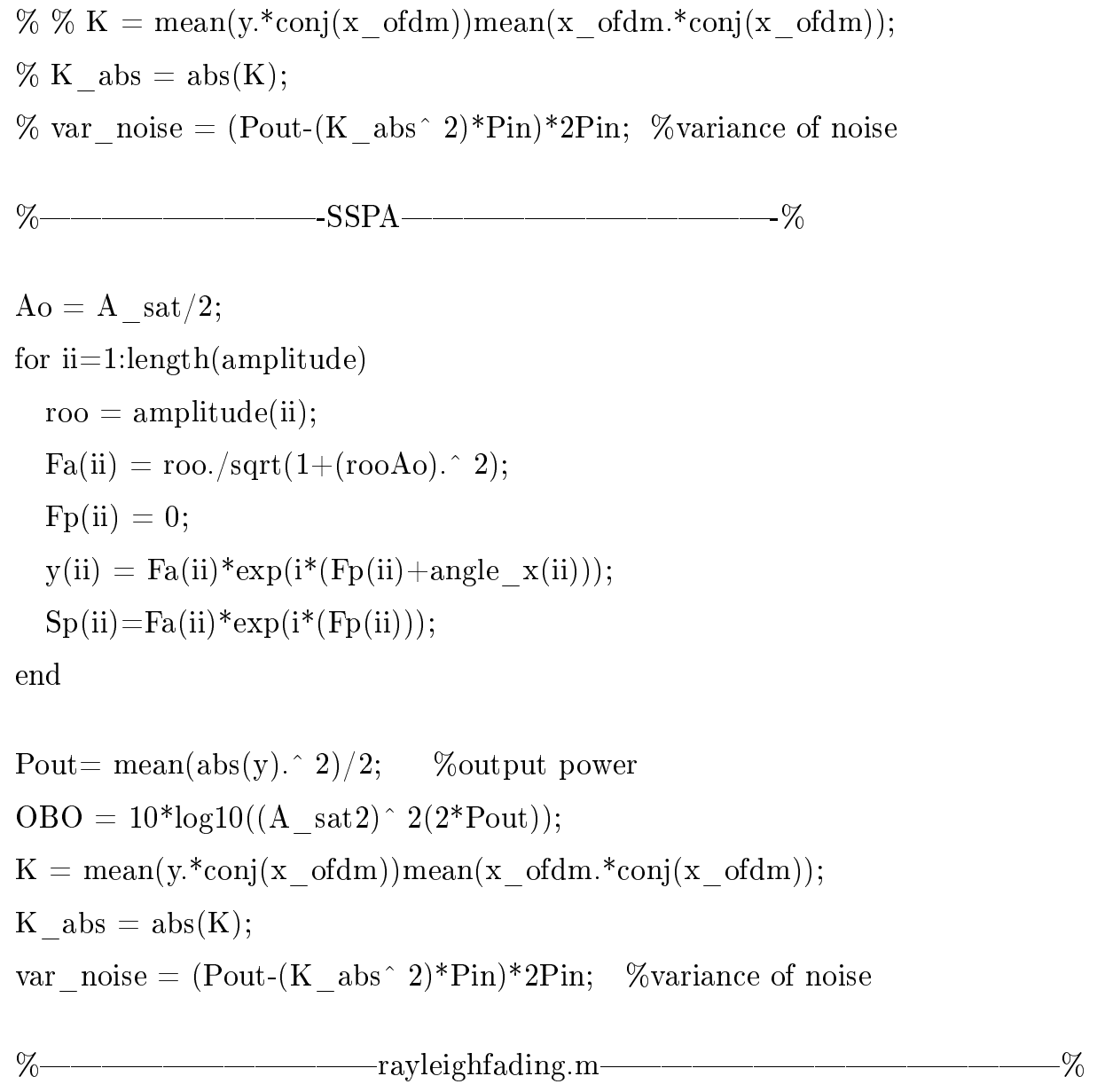

function $[\mathrm{y}, \mathrm{h}]=$ rayleighfading $(\mathrm{x}, \mathrm{t}, \mathrm{snr})$

$\mathrm{Es}=\operatorname{sum}(\operatorname{abs}(\mathrm{x}) \wedge 2) \quad$ length $(\mathrm{x}) ; \quad \%$ power of signal

channel $=\operatorname{sqrt}(12) *\left(\operatorname{randn}(1, \mathrm{t})+\mathrm{i}^{*} \operatorname{randn}(1, \mathrm{t})\right) *\left[\begin{array}{lll}0.5 & 1 & 2\end{array}\right]$;

sigma_n $=$ Es snr $2 ; \quad \%$ noise power

for ii $=1: t$

h(ii) $=$ channel(ii) sum(channel); \%channel tap

end

$\mathrm{y} 1=\operatorname{conv}(\mathrm{h}, \mathrm{x}) ;$

noise $=\operatorname{sqrt}\left(\operatorname{sigma} \_n\right) *\left(\operatorname{randn}(1\right.$, length $(\mathrm{y} 1))+\mathrm{i}^{*} \operatorname{randn}(1$, length $\left.(\mathrm{y} 1))\right)$;

$\mathrm{y}=\mathrm{y} 1+$ noise

$\%$ -phasenoise.m-

function $[\mathrm{y}, \mathrm{w}]=$ phasenoise $(\mathrm{x}, \mathrm{N}, \mathrm{L})$

$\%$ phase noise is modelled as Wiener process 


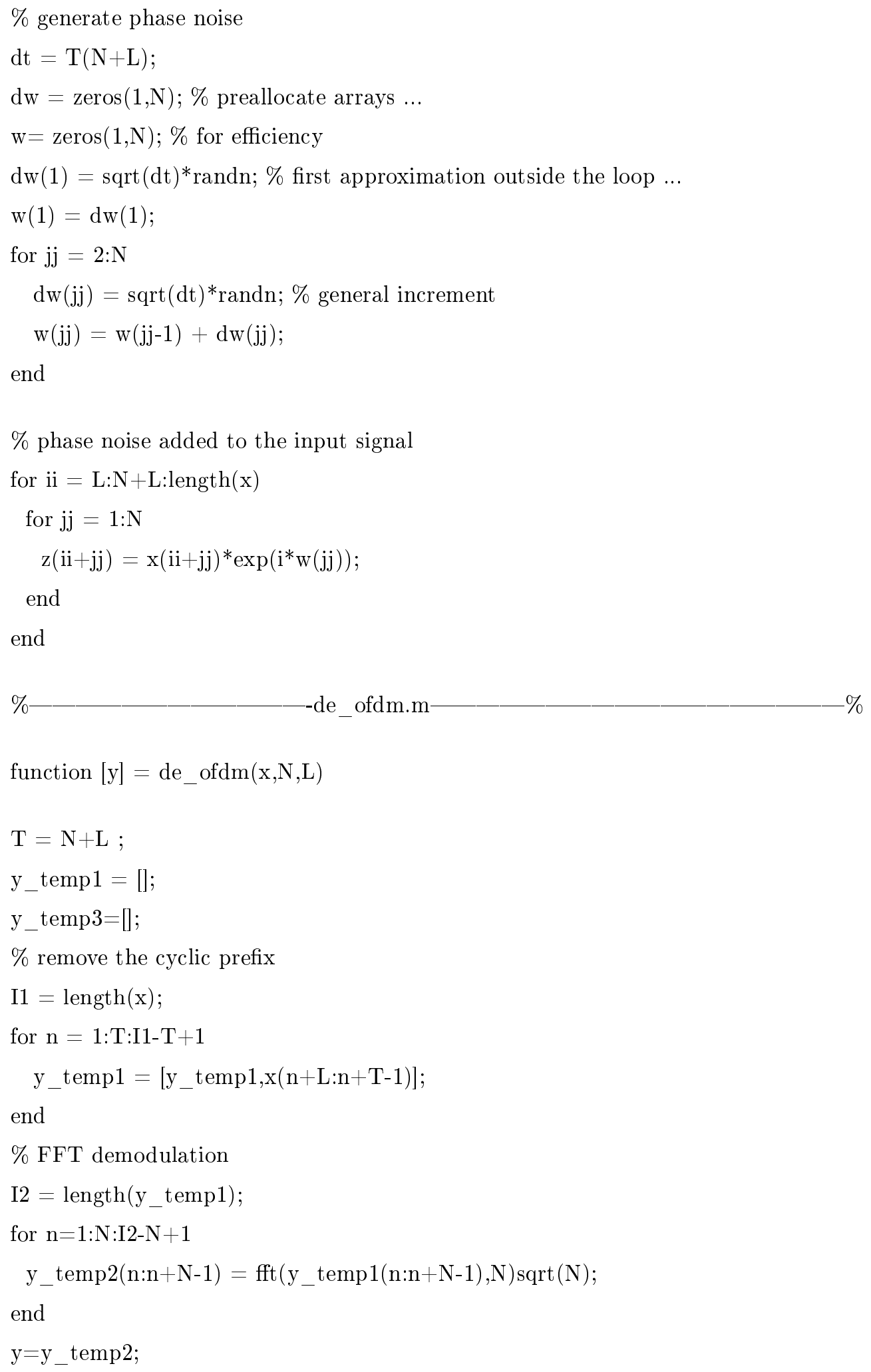

